Alma Mater Studiorum - Università di Bologna

Corso di Laurea Magistrale in Matematica

\title{
REPRESENTATIONS OF SEMISIMPLE LIE ALGEBRAS
}

Tesi di Laurea Magistrale in Algebra

Relatore:

Chiar.ma Prof.ssa

Presentata da:

Nicoletta Cantarini

Sessione I

Anno Accademico 2014-2015 

To Elisa and my family 



\section{Introduction}

This thesis is mainly dedicated to the study of the representations of semisimple Lie algebras over an algebraically closed field $\mathbb{F}$ of characteristic zero.

A semisimple Lie algebra is a finite-dimensional Lie algebra $L$ whose maximal solvable ideal (the radical of $L$ ) is zero. It follows that the quotient of every finite-dimensional Lie algebra with respect to its radical is semisimple. Any simple Lie algebra is, for example, semisimple.

A semisimple Lie algebra $L$ contains a commutative subalgebra $H$, called the Cartan subalgebra of $L$, such that

$$
L=H \oplus \bigoplus_{\alpha \in \Phi} L_{\alpha}
$$

where $\Phi \subset H^{*}$ and $L_{\alpha}$ is the set of common eigenvectors of all the elements $a d_{h}$ for $h \in H$, with eigenfunction $\alpha$. The set $\Phi$, known as the set of roots of the Lie algebra $L$, is a set of roots in the abstract sense [1, pag. 42]. In particular it is possible to write it as a disjoint union of two subsets: $\Phi^{+}$and $\Phi^{-}$, called, respectively, the set of positive and negative roots of $L$.

It turns out that a semisimple Lie algebra decomposes as a direct sum of simple ideals and that simple finite-dimensional Lie algebras are determined, up to isomorphisms, by their root systems.

A representation of a Lie algebra $L$ on a vector space $V$ is a Lie algebra homomorphism of Lie algebras $\varphi: L \rightarrow \mathfrak{g l}(V)$. Equivalently, one can say that $V$ is an $L$-module. An $L$-module is called irreducible if it does not contain any proper submodule and it is called completely reducible if it can be written 
as a direct sum of irreducible submodules. The first fundamental result on the finite-dimensional representations of a semisimple Lie algebra $L$ is due to Weyl [1, pag. 28] and states the complete reducibility of a finite-dimensional $L$-module. Weyl's Theorem thus reduces the study of finite-dimensional $L$ modules to the study of the irreducible ones.

In order to study the representations of a Lie algebra $L$, one can consider the so-called universal enveloping algebra $\mathfrak{U}(L)$ of $L$. This is the associative algebra with unity generated by the elements of $L$. For example, the universal enveloping algebra of the Lie algebra $\mathfrak{s l}_{2}(\mathbb{F})$ can be defined by generators and relations as follows: it is generated by elements $x, y, h$ with defining relations

$$
h x-x h=2 x, \quad h y-y h=-2 y, \quad x y-y x=h .
$$

Every (irreducible) representation of $L$ is an (irreducible) representation of $\mathfrak{U}(L)$ and viceversa. Hence the representation theory of associative algebras comes into play when dealing with the representations of Lie algebras.

A standard cyclic module is a $\mathfrak{U}(L)$-module generated by a maximal vector, i.e., a vector $v$ such that $x_{\alpha} \cdot v=0$ for every $x_{\alpha} \in L_{\alpha}, \alpha \in \Phi^{+}$and $h . v=\lambda(h) v$ for every $h \in H, \lambda \in H^{*}$. The element $\lambda$ is called the weight of $v$. In fact, it turns out that a standard cyclic module $V$ decomposes as a direct sum of common eigenspaces with respect to the action of $H$ - weight spaces - and $\lambda$ is the highest among all weights of $V$, with respect to a partial order. If $V$ is irreducible then its highest weight determines the isomorphism class of $V$ completely. Moreover, the following fundamental result holds:

Theorem 0.0.1. Let $L$ be a semisimple Lie algebra and let $V$ be an irreducible L-module of finite dimension. Then $V$ is isomorphic to an irreducible standard cyclic module of highest weight $\lambda$ for some dominant weight $\lambda$. Viceversa, every irreducible standard cyclic module with dominant highest weight $\lambda$ is finite-dimensional.

Here by a dominant weight we mean an integral function $\lambda \in H^{*}$ such that $\lambda\left(h_{i}\right) \in \mathbb{Z}_{+}$for every $h_{i}$ in a basis of $H$. One can show that this definition does not depend on the choice of the basis. 
In the case of $\mathfrak{s l}_{2}(\mathbb{F})$, this theorem takes a particularly elementary but striking form:

Theorem 0.0.2. The Lie algebra $\mathfrak{s l}_{2}(\mathbb{F})$ has exactly one irreducible representation $\rho$ of each dimension d, up to isomorphism; this representation is realized in the space of homogeneous polynomials of two symbols $X, Y$ of degree $d-1$, and defined by the formulas

$$
\rho(h)=X \frac{\partial}{\partial X}-Y \frac{\partial}{\partial Y}, \quad \rho(x)=X \frac{\partial}{\partial Y}, \quad \rho(y)=Y \frac{\partial}{\partial X} .
$$

The thesis is organized as follows: all the preliminary material is collected and explained in Chapter 1, where almost no proof is given. On the contrary, all the results contained in the rest of the thesis are proved in all details. Chapter 2 is dedicated to Weyl's Theorem and its proof. In Chapter 3 we describe the irreducible $\mathfrak{s l}_{2}(\mathbb{F})$-modules, whose construction is completely carried out by elementary methods. Chapter 4 contains the definition of the universal enveloping algebra of a Lie algebra and the description of its structure. In particular we give a proof of the Poincaré-Birkhoff-Witt Theorem. Chapter 5 contains the construction of the standard cyclic modules and their detailed study, together with the proof of Theorem (0.0.1).

In Chapter 6 we show how the representation theory of Lie algebras naturally applies to the study of Lie superalgebras. A Lie superalgebra $\mathfrak{g}$ is indeed the datum of a Lie algebra $\mathfrak{g}_{\overline{0}}$ (even part) and a $\mathfrak{g}_{\overline{0}}$-module $\mathfrak{g}_{\overline{1}}$ (odd part). The properties of $\mathfrak{g}_{\overline{1}}$ as a $\mathfrak{g}_{\overline{0}}$-module reflect on the structure of $\mathfrak{g}$, for example on its simplicity, as shown in Proposition (6.7.1). Besides, we define a $\mathbb{Z}$-grading on $\mathfrak{g}$ and show how this grading can be used, via representation theory, as a tool to study the structure of the Lie superalgebra $\mathfrak{g}$. The idea of describing Lie (super)algebras as $\mathbb{Z}$-graded (super)algebras has proved to be extremely powerful in the study of both infinite-dimensional Lie algebras and Lie superalgebras. For example one can prove the simplicity of the infinite dimensional Lie algebras of vector fields known as Cartan algebras, using their natural $\mathbb{Z}$-grading (see Section (7.5)). Besides, Kac-Moody algebras, 
i.e., the class of infinite-dimensional Lie algebras closer to the semisimple Lie algebras, were naturally constructed as $\mathbb{Z}$-graded Lie algebras [5].

In Chapter 7 we discuss some interesting examples and explain some important constructions of Lie algebra modules. In particular we prove the simplicity of the Lie algebra of derivations of the algebra of polynomials in $m$ commuting indeterminates and of the Lie superalgebra of derivations of the Grassmann algebra. 


\section{Introduzione}

Questa tesi è dedicata principalmente allo studio delle rappresentazioni di algebre di lie semisemplici su un campo $\mathbb{F}$ algebricamente chiuso di caratteristica zero.

Una algebra di Lie semisemplice è una algebra di Lie $L$ di dimensione finita il cui ideale risolubile massimale (il radicale di $L$ ) è zero. Ne segue che il quoziente di ogni algebra di Lie finito dimensionale rispetto al suo radicale è semisemplice. Ogni algebra di Lie semplice è, ad esempio, semisemplice.

Una algebra di Lie semisemplice $L$ include una sottoalgebra commutativa $H$, chiamata sottoalgebra di Cartan di $L$, tale che

$$
L=H \oplus \bigoplus_{\alpha \in \Phi} L_{\alpha}
$$

dove $\Phi \subset H^{*}$ e $L_{\alpha}$ è l'insieme degli autovettori comuni di tutti gli elementi $a d_{h}$ per $h \in H$, con autofunzione $\alpha$. L'insieme $\Phi$, noto come l'insieme delle radici dell'algebra di Lie $L$, è un insieme di radici in senso astratto [1, pag. 42]. In particolare è possibile scriverlo come unione disgiunta di due insiemi: $\Phi^{+}$e $\Phi^{-}$, chiamati, rispettivamente, l'insieme delle radici positive e negative di $L$.

Si ha che una algebra di Lie semisemplice si decompone in somma diretta di ideali semplici e che le algebre di Lie semplici di dimensione finita sono determinate, a meno di isomorfismo, dai loro sistemi di radici.

Una rappresentazione di una algebra di Lie $L$ su uno spazio vettoriale $V$ è un omomorfismo di algebre di Lie $\varphi: L \rightarrow \mathfrak{g l}(V)$. Equivalentemente si dice che $V$ è un $L$-modulo. Un $L$-modulo è detto irriducibile se non ammette 
sottomoduli propri ed è chiamato completamente riducibile se può essere scritto come somma diretta di sottomoduli irriducibili. Il primo risultato fondamentale sulle rappresentazioni finito dimensionali di una algebra di Lie $L$ si deve a Weyl [1, pag. 28] e afferma la completa riducibilità di ogni $L$ modulo finito dimensionale. Il teorema di Weyl riduce, così, lo studio degli $L$-moduli di dimensione finita allo studio degli $L$-moduli di dimensione finita irriducibili.

Al fine di studiare le rappresentazioni di una algebra di Lie $L$, è necessario considerare la cosiddetta algebra universale inviluppante $\mathfrak{U}(L)$ di $L$. Questa è l'algebra associativa con unità generata dagli elementi di $L$. Per esempio, l'algebra universale inviluppante dell'algebra di Lie $\mathfrak{s l}_{2}(\mathbb{F})$ può essere definita mediante generatori e relazioni nel modo seguente: è generata dagli elementi $x, y, h$ con le relazioni

$$
h x-x h=2 x, \quad h y-y h=-2 y, \quad x y-y x=h .
$$

Ogni rappresentazione (irriducibile) di $L$ è una rappresentazione irriducibile di $\mathfrak{U}(L)$ e viceversa. Quindi, quando si ha a che fare con le rappresentazioni di algebre di Lie, entra in gioco la teoria delle rappresentazioni delle algebre associative.

Un modulo ciclico standard è un $\mathfrak{U}(L)$-modulo generato da un vettore massimale, vale a dire un vettore $v$ tale che $x_{\alpha} \cdot v=0$ per ogni $x_{\alpha} \in L_{\alpha}$, $\alpha \in \Phi^{+}$e $h . v=\lambda(h) v$ per ogni $h \in H, \lambda \in H^{*}$. L'elemento $\lambda$ è chiamato il peso di $v$.

Di fatto risulta che un modulo ciclico standard $V$ si decompone in somma diretta di autospazi comuni rispetto all'azione di $H$ - spazi peso - e $\lambda$ è il più alto tra i pesi di $V$, rispetto a un ordinamento parziale. Se $V$ è irriducibile allora il suo peso più alto determina completamente una classe di isomorfismo di $V$. Inoltre, vale il seguente risultato:

Theorem 0.0.3. Sia $L$ una algebra di Lie semisemplice e $V$ un L-modulo irriducibile di dimensione finita. Allora $V$ è isomorfo a un modulo ciclico standard irriducibile di peso più alto $\lambda$ dove $\lambda$ è un opportuno peso dominante. 
Viceversa, ogni modulo ciclico standard irriducibile di peso più alto $\lambda$, con $\lambda$ peso dominante, ha dimensione finita.

Qui con peso dominante si intende una funzione a valori interi $\lambda \in H^{*}$ tale che $\lambda\left(h_{i}\right) \in \mathbb{Z}_{+}$per ogni $h_{i}$ in una base di $H$. Si può inoltre dimostrare che tale definizione non dipende dalla scelta della base.

Nel caso di $\mathfrak{s l}_{2}(\mathbb{F})$, tale teorema prende una forma particolarmente elementare e potente:

Theorem 0.0.4. L' algebra di Lie $\mathfrak{s l}_{2}(\mathbb{F})$ ha esattamente una rappresentazione irriducibile $\rho$ per ogni dimensione $d$, a meno di isomorfismo; la rappresentazione è data nello spazio dei polinomi omogenei in due simboli $X, Y$ di grado d-1, ed è definita dalle formule

$$
\rho(h)=X \frac{\partial}{\partial X}-Y \frac{\partial}{\partial Y}, \quad \rho(x)=X \frac{\partial}{\partial Y}, \quad \rho(y)=Y \frac{\partial}{\partial X} .
$$

La tesi è organizzata come segue: tutto il materiale preliminare è raggruppato e spiegato nel Capitolo 1, dove non è data pressoché alcuna dimostrazione. Al contrario, tutti i risultati contenuti nel resto della tesi sono dimostrati in ogni dettaglio. Il Capitolo 2 è dedicato al teorema di Weyl e alla sua dimostrazione. Nel Capitolo 3 vengono descritti gli $\mathfrak{s l}_{2}(\mathbb{F})$-moduli, la cui costruzione è ottenuta interamente mediante l'utilizzo di metodi elementari. Nel Capitolo 4 viene data la definizione di algebra universale inviluppante di una algebra di Lie e la descrizione della sua struttura. In particolare è data la dimostrazione del teorema di Poincaré-Birkhoff-Witt. Il Capitolo 5 tratta la costruzione dei moduli ciclici standard e il loro studio dettagliato con la dimostrazione del Teorema (0.0.3).

Nel Capitolo 6 viene mostrato come la teoria delle rappresentazioni si applica naturalmente allo studio delle superalgebre di Lie. Una superalgebra di Lie $\mathfrak{g}$ è, infatti, il dato di una algebra di Lie $\mathfrak{g}_{\overline{0}}$ (parte pari) e di un $\mathfrak{g}_{0^{-}}$ modulo $\mathfrak{g}_{\overline{1}}$ (parte dispari). Le proprietà di $\mathfrak{g}_{\overline{1}}$ come $\mathfrak{g}_{\overline{0}}$-modulo si riflettono sulla struttura di $\mathfrak{g}$, per esempio sulla sua semplicità, come mostrato nella Proposizione (6.7.1). Contemporaneamente, viene definita la $\mathbb{Z}$-graduazione 
su $\mathfrak{g}$ e viene mostrato come questa graduazione può essere usata, tramite la teoria delle rappresentazioni, come strumento per studiare la struttura della superalgebra di Lie $\mathfrak{g}$. L'idea di descrivere le (super)algebre di Lie come (super)algebre $\mathbb{Z}$-graduate si è rivelata estremamente potente nello studio sia delle algebre di Lie infinito dimensionali che nello studio delle superalgebre di Lie. Per esempio si può dimostrare la semplicità delle algebre infinito dimensionali di campi vettoriali note come algebre di Cartan, usando la loro naturale $\mathbb{Z}$-graduazione (guarda la Sezione (7.5)). Ancora, le algebre di KacMoody, cioé, quella classe di algebre di Lie infinto dimensionali più vicine alle algebre di Lie semisemplici, che sono costruite in modo naturale come algebre di Lie $\mathbb{Z}$-graduate [5].

Nel Capitolo 7 vengono discussi alcuni interessanti esempi e vengono spiegati alcune importanti costruzioni di moduli di algebre di Lie. In particolare viene provata la semplicità dell'algebra di Lie delle derivazioni dell'algebra dei polinomi in $m$ indeterminate che commutano e della superalgebra di Lie delle derivazioni dell'algebra di Grassmann. 


\section{Contents}

Introduction $\quad$ i

Introduzione $\quad$ v

1 Preliminaries $\quad 1$

1.1 Preliminary notions and results . . . . . . . . . . . 1

1.2 Roots and weights . . . . . . . . . . . . . 10

1.3 The exponential map . . . . . . . . . . . . . . . . . . 14

2 Weyl's Theorem $\quad 21$

2.1 The Casimir element . . . . . . . . . . . . . . . 21

2.2 Weyl's Theorem . . . . . . . . . . . . . . . . 23

$3 \quad$ Finite dimensional representations of $\mathfrak{s l}_{2}(\mathbb{F}) \quad 27$

3.1 Irreducible finite dimensional representations . . . . . . . . . . 28

4 Universal enveloping algebras 31

4.1 Definition and some basic properties . . . . . . . . . 31

4.2 Poicaré-Birkhoff-Witt Theorem . . . . . . . . . . . . . . . . 34

$5 \quad$ Lie algebras representations $\quad 39$

5.1 Standard cyclic modules . . . . . . . . . . . . . . . 41

5.2 Existence and uniqueness of the irreducible standard cyclic module . . . . . . . . . . . . . . . . . . 45

5.3 Necessary and sufficient condition for finite dimension . . . . . 47 
5.3.1 Necessary condition for finite dimension . . . . . . . 48

5.3.2 Sufficient condition for finite dimension . . . . . . . . . 48

6 Lie superalgebras $\quad 53$

6.1 Superalgebras . . . . . . . . . . . . . . . . 53

6.2 Lie superalgebras . . . . . . . . . . . . . . . . . 54

6.3 The superalgebra $l(V)$ and the supertrace . . . . . . . . 57

6.4 Derivations ..................... . 61

6.5 Linear representations of Lie superalgebras . . . . . . . . . . . 62

$6.6 \mathbb{Z}$-gradings . . . . . . . . . . . . . . . . . . 63

6.7 Criteria of simplicity . . . . . . . . . . . . . . 64

$\begin{array}{lll}7 & \text { Examples } & 67\end{array}$

7.1 Outer tensor product . . . . . . . . . . . . . . . 67

7.2 The action of $\mathfrak{g l}_{n}(\mathbb{F})$ on $\left(\mathbb{F}^{n}\right)^{*} \ldots \ldots \ldots \ldots$

7.3 Example 1: the Lie superalgebra $l(m, n) \ldots \ldots$. . . . . 70

7.4 Example 2: the classical Lie superalgebra $A(m, n) \ldots . . .73$

7.5 Example 3: the Lie algebra $W(m, 0) \ldots \ldots . \ldots 78$

7.6 Example 4: the Lie superalgebra $W(0, n) \ldots \ldots$. . . . . 85

$\begin{array}{lr}\text { Bibliography } & 91\end{array}$ 


\section{Chapter 1}

\section{Preliminaries}

\subsection{Preliminary notions and results}

In this first section some basic notions and fundamental results on Lie algebras are collected. Differently from the rest of the thesis here almost no proof will be given. For more details and all the proofs see [1]. Our main aim is to set the notation and fix the main concepts.

Definition 1.1. An algebra $(A, \cdot)$ over a field $\mathbb{F}$ is a vector space over $\mathbb{F}$ with a product $: A \times A \rightarrow A$. By product we mean a bilinear map.

Definition 1.2. An algebra $(A, \cdot)$ is called associative if $\cdot$ is an associative product, i.e.

$$
(a \cdot b) \cdot c=a \cdot(b \cdot c) .
$$

Example 1.1. The field $\mathbb{F}$ with its product is an associative algebra.

Example 1.2. The ring of polynomials $\mathbb{F}[\mathrm{x}]$ with the usual product of polynomials is an associative algebra.

Example 1.3. The set of matrices $M_{n, n}(\mathbb{F})$ with the product row per column is an associative algebra. 
Example 1.4. Given a vector space $V$ the linear space $E n d(V)$ of endomorphisms of $V$ with the usual composition o of endomorphisms is an associative algebra.

Definition 1.3. A Lie algebra is an algebra $L$ whose product [, ] : $L \times L \rightarrow L$ satisfies the following properties:

$$
\begin{array}{r}
{[x, x]=0} \\
{[x,[y, z]]=[[x, y], z]+[y,[x, z]] .}
\end{array}
$$

Remark 1. Properties (1.1) and (1.2) applied to $[x+y, x+y]$ imply the anticommutativity of the product:

$$
[x, y]=-[y, x]
$$

Conversely, if $\mathbb{F}$ has not characteristic 2, property (1.3) implies property (1.1).

Example 1.5. Given an associative algebra $(A, \cdot)$, we can define on $A$ a Lie algebra structure by setting:

$$
[a, b]=a \cdot b-b \cdot a
$$

We will call $(A,[]$,$) , the Lie algebra associated to (A, \cdot)$ and we will denote it simply by $A_{L}$.

Example 1.6. $\mathbb{F}_{L}$ is a Lie algebra.

Example 1.7. We will denote by $\mathfrak{g l}_{n}(\mathbb{F})$ the Lie algebra associated to $\left(M_{n, n}(\mathbb{F}), \cdot\right)$. Similarly we will denote by $\mathfrak{g l}(V)$ the Lie algebra associated to $(\operatorname{End}(V), \circ)$.

Example 1.8. The subalgebra of $\mathfrak{g l}_{n}(\mathbb{F})$ of matrices with 0-trace is a Lie algebra which we will denote by $\mathfrak{s l}_{n}(\mathbb{F})$.

Definition 1.4. Given an algebra $A$, a linear map $D: A \rightarrow A$ is called a derivation of $A$ if it satisfies the so-called Leibniz rule, i.e.

$$
D(a \cdot b)=D(a) \cdot b+a \cdot D(b) .
$$


Remark 2. Let $D$ be a derivation of $A$. Then $D(1)=0$.

Proof. $D(1)=D(1 \cdot 1)=D(1) \cdot 1+1 \cdot D(1)=2 D(1)$. Therefore $D(1)=0$.

Remark 3. We denote by $\operatorname{der}(A)$ the subset of $\operatorname{End}(A)$ consisting of the derivations of $A$. Then $\operatorname{der}(A)$ is a Lie subalgebra of $\operatorname{End}(A)_{L}$.

Proof. Let $D, D^{\prime}$ be two derivations of $A$.

$$
\begin{array}{r}
{\left[D, D^{\prime}\right](a \cdot b)=\left(D \circ D^{\prime}-D^{\prime} \circ D\right)(a \cdot b)=} \\
D\left(D^{\prime}(a \cdot b)\right)-D^{\prime}(D(a \cdot b))= \\
D\left(D^{\prime}(a) \cdot b+a \cdot D^{\prime}(b)\right)-D^{\prime}(D(a) \cdot b+a \cdot D(b))= \\
D\left(D^{\prime}(a)\right) \cdot b+D^{\prime}(a) \cdot D(b)+D(a) \cdot D^{\prime}(b)+a \cdot D\left(D^{\prime}(b)\right)- \\
D^{\prime}(D(a)) \cdot b-D(a) \cdot D^{\prime}(b)-D^{\prime}(a) \cdot D(b)-a \cdot D^{\prime}(D(b))= \\
D\left(D^{\prime}(a)\right) \cdot b+a \cdot D\left(D^{\prime}(b)\right)-D^{\prime}(D(a)) \cdot b-a \cdot D^{\prime}(D(b))= \\
D \circ D^{\prime}(a) \cdot b+a \cdot D \circ D^{\prime}(b)-D^{\prime} \circ D(a) \cdot b-a \cdot D^{\prime} \circ D(b)= \\
{\left[D, D^{\prime}\right](a) \cdot b+a \cdot\left[D, D^{\prime}\right](b) .}
\end{array}
$$

Therefore $\left[D, D^{\prime}\right]$ is a derivation.

Example 1.9. We set $W(m, 0)=\operatorname{der}\left(\mathbb{F}\left[x_{1}, \ldots, x_{m}\right]\right)$.

From now on, when dealing with associative algebras, we will omit the symbol of the product.

Let $L$ be a Lie algebra over a field $\mathbb{F}$.

Definition 1.5. A subspace $K$ of $L$ is called a Lie subalgebra of $L$ if $[x, y] \in$ $K$ whenever $x, y \in K$.

Definition 1.6. A subspace $I$ of $L$ is called an ideal of $L$ if for all $x \in I$ and for all $y \in L,[x, y]$ lies in $I$.

Example 1.10. 0 is an ideal of $L$.

Example 1.11. $Z(L)=\{x \in L \mid[x, y]=0$ for all $y \in L\}$ is an ideal of $L$. 
Example 1.12. $[L, L]=\langle[x, y]: x, y \in L\rangle$ is an ideal of $L$.

Definition 1.7. A Lie algebra $L$ is said to be simple if $L$ has no ideals except itself and 0 and if moreover $[L, L] \neq 0$.

Definition 1.8. A linear map $\phi: L \rightarrow L^{\prime}$, where $L, L^{\prime}$ are Lie algebras, is called a homomorphism of Lie algebras if $\phi([x, y])=[\phi(x), \phi(y)]$ for all $x, y \in L . \phi$ is called an isomorphism of Lie algebras if $\phi$ is an isomorphism of vector spaces and a homomorphism of Lie algebras.

Example 1.13. Given an element $x \in L$, the linear map $a d_{x}: L \rightarrow L$ such that $a d_{x}(y)=[x, y]$ is a derivation of $L$. Moreover the linear map $a d: L \rightarrow \mathfrak{g l}(L), \operatorname{ad}(x)=a d_{x}$ is a homomorphism of Lie algebras.

Definition 1.9. A Lie algebra $L$ is said to be nilpotent if there exists some $i \in \mathbb{Z}_{+}$such that $L^{i}=0$ where $L^{0}=L$ and $L^{i}=\left[L^{i-1}, L\right]$ for $i>0$.

Remark 4. If $L$ is nilpotent, then, for every $x \in L, a d_{x}$ is nilpotent and we say that $x$ is $a d$-nilpotent.

The following theorem holds:

Theorem 1.1.1 (Engel's Theorem). If all elements of $L$ are ad-nilpotent, then $L$ is nilpotent.

For a proof see [1, pagg. 12-13].

Definition 1.10. A Lie algebra $L$ is said to be solvable if there exists some $i$ such that $L^{(i)}=0$ where $L^{(0)}=L$ and $L^{(i)}=\left[L^{(i-1)}, L^{(i-1)}\right]$ for $i>0$.

Remark 5. If $L$ is nilpotent then it is solvable.

Remark $6 . L$ is solvable if and only if $[L, L]$ is nilpotent.

Remark 7. Every finite dimensional Lie algebra has a maximal solvable ideal. It is called the radical of $L$ and it is denoted by $\operatorname{Rad}(L)$.

Definition 1.11. A Lie algebra $L$ is said to be semisimple if $\operatorname{Rad}(L)=0$. 
Remark 8. $L / \operatorname{Rad}(L)$ is semisimple.

The following theorem holds:

Theorem 1.1.2 (Lie's Theorem). Let $L$ be a solvable subalgebra of $\mathfrak{g l}(V), V$ finite dimensional. If $V \neq 0$, then $V$ contains a common eigenvector for all the endomorphisms in $L$.

For a proof see [1, pagg 15-16].

Definition 1.12. A representation of a Lie algebra $L$ on a vector space $V$ is a homomorphism $\phi: L \rightarrow \mathfrak{g l}(V)$.

Example 1.14. Let $L$ be a Lie algebra. Then the map $\phi: L \rightarrow \mathfrak{g l}(\mathbb{F})$ defined by $\phi(x)=0$ for all $x \in L$ is a representation of $L$ called the trivial representation of $L$.

Example 1.15. The map $\phi: \mathfrak{g l}_{n}(\mathbb{F}) \rightarrow \mathfrak{g l}\left(\mathbb{F}^{n}\right)$ defined by $\phi(x)=x$, is a representation of $\mathfrak{g l}_{n}(\mathbb{F})$ on $\mathbb{F}^{n}$ called the standard representation of $\mathfrak{g l}_{n}(\mathbb{F})$. Similarly the map $\phi: \mathfrak{s l}_{n}(\mathbb{F}) \rightarrow \mathfrak{s l}\left(\mathbb{F}^{n}\right)$ defined by $\phi(x)=x$, is a representation of $\mathfrak{s l}_{n}(\mathbb{F})$ on $\mathbb{F}^{n}$ called the standard representation of $\mathfrak{s l}_{n}(\mathbb{F})$

Example 1.16. For every Lie algebra $L$ the map ad $L \rightarrow \mathfrak{g l}(L)$ is a representation of $L$ on itself called the adjoint representation.

Definition 1.13. A representation is said to be faithful if it is an injective map.

The following theorems hold:

Theorem 1.1.3 (Cartan's Criterion). Let $L$ be a subalgebra of $\mathfrak{g l}(V)$ where $V$ is a finite dimensional vector space. If $\operatorname{tr}(x y)=0$ for all $x \in[L, L]$ and $y \in L$. Then $L$ is solvable.

Corollary 1.1.4. Let $L$ be a Lie algebra such that $\operatorname{tr}\left(a d_{x} a d_{y}\right)=0$ for all $x \in[L, L]$ and $y \in L$. Then $L$ is solvable. 
For proofs see [1, pag. 20].

Definition 1.14. A vector space $V$ endowed with an operation

$$
\begin{array}{r}
.: L \times V \rightarrow V \\
\quad(x, y) \mapsto x . y,
\end{array}
$$

is said to be an $L$-module if the following conditions are satisfied:

1. $(a x+b y) \cdot v=a(x \cdot v)+b(y \cdot v)$,

2. $x .(a v+b w)=a(x . v)+b(x . w)$,

3. $[x, y] . v=x . y . v-y . x . v$

for all $x, y \in L, v, w \in V, a, b \in \mathbb{F}$.

Remark 9. Having an $L$-module $V$ is equivalent to having a representation of $L$ on $V$. If $\phi: L \rightarrow \mathfrak{g l}(V)$ is a representation of $L$ on $V$ we can define a structure of $L$-module on $V$ as follows:

$$
x . v=\phi(x)(v) .
$$

On the other hand, if $V$ is an $L$-module, then we can define a representation of $L$ on $V$ as follows:

$$
\phi(x)(v)=x \cdot v .
$$

Example 1.17. If $V$ is an $L$-module, then we can define an action of $L$ on $V^{*}$, that turns $V^{*}$ into an $L$-module, as follows:

$$
(x . \varphi)(v)=-\varphi(x . v)
$$

where $x \in L, \varphi \in V^{*}$ and $v \in V$.

Example 1.18. If $V, W$ are two $L$-modules, then we can define the following action of $L$ on $V \otimes W$, that turns $V \otimes W$ into an $L$-module:

$$
x .(v \otimes w)=(x . v) \otimes w+v \otimes(x . w)
$$

where $x \in L, v \in V$ and $w \in W$. 
Example 1.19. If $V, W$ are two $L$-modules, then we can define an action of $L$ on the space $\operatorname{Hom}(V, W)$ of linear applications from $V$ into $W$, that turns $\operatorname{Hom}(V, W)$ into an $L$-module, as follows:

$$
(x . \varphi)(v)=-\varphi(x . v)+x \cdot(\varphi(v))
$$

where $x \in L, \varphi \in H o m(V, W)$ and $v \in V$.

Definition 1.15. A linear map $\phi: V \rightarrow V^{\prime}$, where $V, V^{\prime}$ are $L$-modules, is called a homomorphism of $L$-modules if $\phi(x . v)=x . \phi(v)$ for all $x \in L$ and $v \in V$.

Remark 10. The kernel of a homomorphism $\phi: V \rightarrow W$ of $L$-modules is an $L$-submodule of $V$.

Definition 1.16. An $L$-module $V$ is called irreducible if it has only two $L$-submodules: itself and 0 .

Example 1.20. The trivial representation of $L$ is irreducible.

Example 1.21. The standard representation of $\mathfrak{g l}_{n}(\mathbb{F})$ and the standard representation of $\mathfrak{s l}_{n}(\mathbb{F})$ are irreducible because $\mathfrak{g l}_{n}(\mathbb{F})$ and $\mathfrak{s l}_{n}(\mathbb{F})$ act in a transitive way on $\mathbb{F}^{n}$.

Example 1.22. The adjoint representation of $L$ is irreducible if and only if $L$ is simple.

Example 1.23. Let $V$ be a $\mathfrak{g l}_{n}(\mathbb{F})$-module. Then $V$ is an irreducible $\mathfrak{g l}_{n}(\mathbb{F})$ module if and only if $V$ is an irreducible $\mathfrak{s l}_{n}(\mathbb{F})$-module because $\mathfrak{g l}_{n}(\mathbb{F})=$ $\mathfrak{s l}_{n}(\mathbb{F}) \oplus\left\langle 1_{n}\right\rangle$.

Example 1.24. If $V$ is an $L$-module of dimension 1, then $V$ is an irreducible $L$-module.

Proposition 1.1.5. Let $V$ a be a finite dimensional L-module. $V$ is irreducible if and only if the L-module $V^{*}$ is irreducible. 
Proof. Let us prove that if $V^{*}$ is an irreducible $L$-module, then $V$ is an irreducible $L$-module. Let us suppose, by contradiction, that $V$ is not irreducible, hence there exists a proper $L$-submodule $V_{1}$ of $V$. Let us define $V^{1}=\left\{\varphi \in V^{*} \mid \varphi(v)=0\right.$ for all $\left.v \in V_{1}\right\}$. Let $\left\{v_{1}, \ldots, v_{k}\right\}$ be a basis of $V_{1}$ and let us complete $\left\{v_{1}, \ldots, v_{k}\right\}$ to a basis $\left\{v_{1}, \ldots, v_{k}, v_{k+1}, \ldots, v_{n}\right\}$ of $V$. Let, then, $\left\{\varphi_{1}, \ldots, \varphi_{n}\right\}$ be the dual basis of $V^{*}$. Note that $V^{1} \neq 0$ because $\varphi_{j}\left(v_{i}\right)=0$ for all $j>k$ and $i \leq k$, and $V^{1} \neq V^{*}$ because $\varphi_{1}\left(v_{1}\right)=1 \neq 0$. Moreover $V^{1}$ is an $L$-submodule of $V^{*}$ because $(x . \varphi)(v)=-\varphi(x . v)=0$ for all $\varphi \in V^{1}, x \in L$ and $v \in V_{1}$, hence $V^{1}$ is a proper $L$-submodule of $V^{*}$, but this is a contradiction because $V^{*}$ is irreducible. Let us now prove that if $V$ is an irreducible $L$-module, then $V^{*}$ is an irreducible $L$-module. Let us consider the canonical isomorphism $\beta: V \rightarrow V^{* *}$ defined as follows, for $v \in V:$

$$
\beta(v): V^{*} \rightarrow \mathbb{F}, \beta(v)(\varphi)=\varphi(v) .
$$

$\beta$ is a homomorphism of $L$-modules indeed $\beta(x . v)(\varphi)=\varphi(x . v)=-(x . \varphi)(v)=$ $-\beta(v)(x . \varphi)=(x . \beta(v))(\varphi)$ hence $\beta(x . v)=x \cdot \beta(v)$ and $\beta$ is an isomorphism of $L$-modules. Since $\beta$ is an isomorphism of $L$-modules, $V^{* *}$ is an irreducible $L$-module, therefore, thanks to what we proved above, $V^{*}$ is an irreducible $L$-module.

Definition 1.17. An $L$-module $V$ is said to be completely reducible if it is a direct sum of irreducible $L$-submodules or, equivalently, if each $L$-submodule $W$ of $V$ has a complement $W^{\prime}$, i.e. there exists an $L$-submodule $W^{\prime}$ such that $V=W \oplus W^{\prime}$.

Let now $L$ be a semisimple Lie algebra over an algebraically closed field with characteristic 0 . An element $x \in L$ is said to be semisimple if $a d_{x}$ is semisimple.

Definition 1.18. A subalgebra of semisimple elements is called a toral subalgebra of $L$.

Every finite-dimensional semisimple Lie algebra $L$ has a toral subalgebra. And every toral subalgebra is abelian. 
Definition 1.19. A subalgebra of a Lie algebra $L$ is called a maximal toral subalgebra of $L$ if it is a toral subalgebra not properly included in any other toral subalgebra.

Remark 11. Since $H$ is abelian $a d_{L} H$ consists of commuting semisimple endomorphisms of $L$ hence the elements in $a d_{L} H$ are simultaneously diagonalizable. As a consequence

$$
L=\oplus L_{\alpha}
$$

where $L_{\alpha}=\{x \in L \mid[h, x]=\alpha(h) x$ for all $h \in H\}$ with $\alpha \in H^{*}$.

The set of nonzero $\alpha \in H^{*}$ such that $L_{\alpha} \neq 0$ is denoted by $\Phi$ and its elements are called the roots of $L$ relative to $H$. $L_{0}=C_{L}(H)$. Moreover the following proposition holds:

Proposition 1.1.6. Let $H$ a maximal toral subalgebra of $L$. Then $H=$ $C_{L}(H)$.

For a proof see [1, pagg. 36-37].

Due to Proposition (1.1.6) the following decomposition, known as the Cartan decomposition of $L$, holds:

$$
L=H \oplus \bigoplus_{\alpha \in \Phi} L_{\alpha}
$$

Example 1.25. Let us consider a basis of $\mathfrak{s l}_{2}(\mathbb{F})$ given by $\{x, y, h\}$ where

$$
x=\left(\begin{array}{ll}
0 & 1 \\
0 & 0
\end{array}\right), y=\left(\begin{array}{ll}
0 & 0 \\
1 & 0
\end{array}\right), h=\left(\begin{array}{cc}
1 & 0 \\
0 & -1
\end{array}\right)
$$

then $[x, y]=h,[h, x]=2 x,[h, y]=-2 y$. It follows:

$$
\mathfrak{s l}_{2}(\mathbb{F})=\langle h\rangle \oplus \mathfrak{s l}_{2}(\mathbb{F})_{\alpha} \oplus \mathfrak{s l}_{2}(\mathbb{F})_{-\alpha}
$$

where $\alpha \in\langle h\rangle^{*}$ such that $\alpha(h)=2, \mathfrak{s l}_{2}(\mathbb{F})_{\alpha}=\langle x\rangle$ and $\mathfrak{s l}_{2}(\mathbb{F})_{-\alpha}=\langle y\rangle$.

Example 1.26. Let $e_{i j}$, with $i, j \in\{1, \ldots, n\}$, be the matrix of $M_{n, n}(\mathbb{F})$ such that $\left(e_{i j}\right)_{r s}=\delta_{i r} \delta_{j s}$. A maximal toral subalgebra of $\mathfrak{s l}_{n}(\mathbb{F})$ is $H=$ diagonal 
matrices of $M_{n, n}(\mathbb{F})$ with 0 -trace $\}=\left\langle e_{i i}-e_{i+1 i+1}: 1 \leq i \leq n-1\right\rangle$. Since, for $h=\sum_{k=1}^{n} h_{k} e_{k k}$ and $i \neq j$,

$$
\begin{array}{r}
{\left[h, e_{i j}\right]=} \\
{\left[\sum_{k=1}^{n} h_{k} e_{k k}, e_{i j}\right]=} \\
\sum_{k=1}^{n} h_{k}\left[e_{k k}, e_{i j}\right]=\sum_{k=1}^{n} h_{k}\left(\delta_{k i} e_{k j}-\delta_{j k} e_{i k}\right)= \\
\left(h_{i}-h_{j}\right) e_{i j},
\end{array}
$$

the root system of $\mathfrak{s l}_{n}(\mathbb{F})$ with respect to $H$ is

$$
\Phi=\left\{\alpha_{i j} \in H^{*} \mid \alpha_{i j}\left(\sum_{k=1}^{n} h_{k} e_{k k}\right)=h_{i}-h_{j}, i \neq j\right\}
$$

and $\mathfrak{s l}_{n}(\mathbb{F})_{\alpha_{i j}}=\left\langle e_{i j}\right\rangle$. Therefore

$$
\mathfrak{s l}_{n}(\mathbb{F})=H \oplus \bigoplus_{\alpha \in \Phi} \mathfrak{s l}_{n}(\mathbb{F})_{\alpha}
$$

with $\mathfrak{s l}_{n}(\mathbb{F})_{\alpha_{i j}}=\left\langle e_{i j}\right\rangle$.

Proposition 1.1.7. Let $x_{\alpha} \in L_{\alpha}, x_{\alpha} \neq 0$. Then there exists $y_{\alpha} \in L_{-\alpha}$ such that

$$
\left\langle x_{\alpha}, y_{\alpha},\left[x_{\alpha}, y_{\alpha}\right]=h_{\alpha}\right\rangle \cong \mathfrak{s l}_{2}(\mathbb{F})
$$

and we denote $\left\langle x_{\alpha}, y_{\alpha},\left[x_{\alpha}, y_{\alpha}\right]=h_{\alpha}\right\rangle$ by $\mathfrak{s l}_{2}(\alpha)$.

\subsection{Roots and weights}

Definition 1.20. Given a euclidean space $E$, i.e. a finite dimensional vector space over $\mathbb{R}$, endowed with a positive definite symmetric bilinear form $($,$) ,$ we can define $\langle\alpha, \beta\rangle=2 \frac{(\alpha, \beta)}{(\beta, \beta)}$.

Remark 12 . Note that $\langle$,$\rangle is linear only in the first variable.$

Given a nonzero vector $\alpha \in E$ we can define a reflection $\sigma_{\alpha}$, with reflecting hyperplane $P_{\alpha}=\{\beta \in E \mid(\beta, \alpha)=0\}$, as follows: $\sigma_{\alpha}(\beta)=\beta-\langle\beta, \alpha\rangle \alpha$. 
Definition 1.21. A subset $\tilde{\Phi}$ of a euclidean space $E$ is called a root system in $E$ if the following properties are satisfied:

1. $\tilde{\Phi}$ is finite, spans $E$ and does not contain 0 .

2. If $\alpha \in \tilde{\Phi}$ the only multiple scalars of $\alpha$ in $\tilde{\Phi}$ are $\alpha$ and $-\alpha$.

3. If $\alpha, \beta \in \tilde{\Phi}$ then $\sigma_{\alpha}(\beta)$ lies in $\tilde{\Phi}$.

4. If $\alpha, \beta \in \tilde{\Phi}$, then $\langle\beta, \alpha\rangle \in \mathbb{Z}$.

Remark 13. The set $\Phi$ of the roots of a semisimple Lie algebra $L$ with respect to a maximal toral subalgebra $H$ of $L$ is a root system, i.e. it satisfies Definition (1.21)

Definition 1.22. We call the subgroup of $O(E)$ generated by the reflections $\sigma_{\alpha}$ with $\alpha \in \Phi$ the Weyl group and we denote it by $\mathcal{W}$.

Definition 1.23. A subset $\Delta$ of $\Phi$ is called a base of $\Phi$ if the following properties hold:

1. $\Delta$ is a basis of $E$.

2. Each root $\beta$ can be written as $\beta=\sum k_{\alpha} \alpha$ with $\alpha \in \Delta$ and $k_{\alpha} \in \mathbb{Z}$ all nonnegative or nonpositive.

We denote by $\Phi^{+}$the set of all roots in $\Phi$ which can be written as a sum of elements in $\Delta$ with nonnegative integral coefficients (positive roots) and we denote by $\Phi^{-}$the set of all roots in $\Phi$ which can be written as a sum of elements in $\Delta$ with nonpositive integral coefficients (negative roots).

Example 1.27. Let us consider the Lie algebra $\mathfrak{s l}_{n}(\mathbb{F})$. As we have already seen, $\Phi=\left\{\alpha_{i j} \in H^{*} \mid \alpha_{i j}\left(\sum_{k=1}^{n} h_{k} e_{k k}\right)=h_{i}-h_{j}, i \neq j\right\}$, then $\Delta=\left\{\alpha_{i}=\right.$ $\left.\alpha_{i i+1} \mid 1 \leq i \leq n-1\right\}$ is a base of $\Phi$, indeed, if $i<j, \alpha_{i j}=\alpha_{i}+\alpha_{i+1}+\ldots+\alpha_{j-1}$ and, if $i>j, \alpha_{i j}=-\alpha_{j i}=-\left(\alpha_{j}+\alpha_{j+1}+\ldots+\alpha_{i-1}\right)$. It follows:

- $\Phi^{+}=\left\{\alpha_{i j}: i<j\right\}$ 
- $\Phi^{-}=\left\{\alpha_{i j}: i>j\right\}$.

The following theorem holds:

Theorem 1.2.1. Every root system $\Phi$ has a base.

For a proof see [1, pagg. 48-49].

Definition 1.24. Let $\lambda$ be in $E$. $\lambda$ is called a weight if $\langle\lambda, \alpha\rangle \in \mathbb{Z}$ for every $\alpha \in \Phi$. The set of all weights is denoted by $\Lambda$.

Remark 14 . Let $\lambda$ be in $E$. $\lambda$ is a weight if and only if $\langle\lambda, \alpha\rangle \in \mathbb{Z}$ for every $\alpha \in \Delta$.

Before giving a proof of the Remark (14) we recall that:

$$
\Phi^{V}=\left\{\frac{2 \alpha}{(\alpha, \alpha)}, \alpha \in \Phi\right\}
$$

is still a root system and $\Delta^{V}=\left\{\alpha^{V}, \alpha \in \Delta\right\}$ is a base for $\Phi^{V}$.

Proof. $\lambda$ is a weight if and only if, by definition, $\langle\lambda, \alpha\rangle \in \mathbb{Z}$ for all $\alpha \in \Phi$, that is if and only if $\left(\lambda, \alpha^{V}\right) \in \mathbb{Z}$ for all $\alpha^{V} \in \Phi^{V}$ so, if and only if $\left(\lambda, \alpha^{V}\right) \in \mathbb{Z}$ for all $\alpha^{V} \in \Delta^{V}$ because (, ) is bilinear and $\Delta^{V}$ a base for $\Phi^{V}$. But $\left(\lambda, \alpha^{V}\right)=\langle\lambda, \alpha\rangle$. So we have proved that $\lambda$ is a weight if and only if $\langle\lambda, \alpha\rangle \in \mathbb{Z}$ for all $\alpha \in \Delta$.

Definition 1.25. We say that $\lambda$ is dominant if $\langle\lambda, \alpha\rangle \geq 0$ for all $\alpha \in \Phi^{+}$ and we denote the set of dominant weights by $\Lambda^{+}$. We say that $\lambda$ is strongly dominant if $\langle\lambda, \alpha\rangle>0$ for all $\alpha \in \Phi^{+}$.

Let $\Delta$ be $\left\{\alpha_{1}, \ldots, \alpha_{l}\right\}$ and let $\left\{\lambda_{1}, \ldots, \lambda_{l}\right\}$ be its dual basis with respect to $\langle$, $\rangle$, i.e. $\left\langle\lambda_{i}, \alpha_{j}\right\rangle=\delta_{i j}$.

Proposition 1.2.2. $\Lambda$ is a lattice with basis $\left\{\lambda_{1}, \ldots, \lambda_{l}\right\}$.

Proof. Let $\lambda$ be a weight. Then $\left\langle\lambda, \alpha_{i}\right\rangle=m_{i} \in \mathbb{Z}$. Let us consider $\lambda^{\prime}=$ $\sum_{i=1}^{l} m_{i} \lambda_{i}$. Then $\left\langle\lambda^{\prime}, \alpha_{i}\right\rangle=\sum_{j=1}^{l} m_{j}\left\langle\lambda_{j}, \alpha_{i}\right\rangle=\sum_{j=1}^{l} m_{j} \delta_{i j}=m_{i}$. Thus $\lambda=\lambda^{\prime}$ because $($,$) is nondegenerate and \left(\lambda-\lambda^{\prime}, \alpha_{i}^{V}\right)=0$ for all $i$. 
Remark 15. Note that $\Phi \subseteq \Lambda$ and $\alpha_{i}=\sum_{j=1}^{l} m_{i, j} \lambda_{i}$ where $m_{i, j}=\left\langle\alpha_{i}, \alpha_{j}\right\rangle$, i.e. the Cartan matrix expresses the change of basis between $\left\{\lambda_{1}, \ldots, \lambda_{l}\right\}$ and $\left\{\alpha_{1}, \ldots, \alpha_{l}\right\}$.

Remark 16. $\mathcal{W}$ acts on $\Lambda$, i.e. $w(\lambda) \in \Lambda$ for all $w \in \mathcal{W}$ and $\lambda \in \Lambda$.

Proof. Let $\lambda \in \Lambda$. Then $\langle w(\lambda), \alpha\rangle=\frac{2(w(\lambda), \alpha)}{(\alpha, \alpha)}=\frac{2\left(\lambda, w^{-1}(\alpha)\right)}{\left(w^{-1}(\alpha), w^{-1}(\alpha)\right)}=\left\langle\lambda, w^{-1}(\alpha)\right\rangle \in$ $\mathbb{Z}$.

We can define an ordering on the set of weights:

Definition 1.26. Let $\mu, \lambda$ be two elements of $\Lambda$. Then we write $\mu \prec \lambda$ if we can write $\lambda=\mu+\sum a_{i} \alpha_{i}$ with $a_{i} \in \mathbb{Z}_{+}$and $\alpha_{i} \in \Delta$.

Remark 17. Note that $\prec$ is not a total ordering on $\Lambda$. Indeed if $\Delta=$ $\left\{\alpha_{1}, \ldots, \alpha_{l}\right\}$ is a base of a root system $\Phi$, by Remark (15), $\Phi \subseteq \Lambda$ and neither $\alpha_{1} \prec \alpha_{2}$ nor $\alpha_{2} \prec \alpha_{1}$.

Proposition 1.2.3. Every weight is conjugated under $\mathcal{W}$ to one and only one dominant weight. If $\lambda$ is dominant, then $w(\lambda) \prec \lambda$ for all $w \in \mathcal{W}$.

For a proof see [1, pag. 52, Lemma B].

Remark 18. A dominant weight is the maximal element with respect to $\prec$ in the $\mathcal{W}$-orbit where it lies.

Remark 19. The maximal weight in a $\mathcal{W}$-orbit is a dominant weight, but it can happen that there exists a weight $\lambda$ which is not dominant and a dominant weight $\mu$ such that $\mu \prec \lambda$. In this case Proposition (1.2.3) implies that $\lambda$ is not $\mathcal{W}$-conjugated with $\mu$.

Lemma 1.2.4. Let $\lambda$ be in $\Lambda^{+}$. Then $\left\{\mu \in \Lambda^{+}\right\} \cap\{\mu: \mu \prec \lambda\}$ is finite.

Proof. Let $\mu$ be a dominant weight such that $\mu \prec \lambda$, i.e. $\lambda-\mu=\sum a_{i} \alpha_{i}$ with $a_{i} \in \mathbb{Z}^{+}$. Then $\lambda+\mu$ is dominant, because $\langle\lambda+\mu, \alpha\rangle=\langle\lambda, \alpha\rangle+\langle\mu, \alpha\rangle$ and these two summands are nonnegative for all $\alpha \in \Phi^{+}$. We have $0 \leq$ $(\lambda+\mu, \lambda-\mu)=(\lambda, \lambda)-(\mu, \mu)$ thus $(\mu, \mu) \leq(\lambda, \lambda)$ hence there is a finite number of possibilities because the set of weights is a lattice. 


\subsection{The exponential map}

Definition 1.27. Let $x$ be an $a d$-nilpotent element of a Lie algebra $L$. Then

$$
\exp \left(a d_{x}\right)=\sum_{i=0}^{\infty} \frac{\left(a d_{x}\right)^{i}}{i !}=1+a d_{x}+\frac{1}{2}\left(a d_{x}\right)^{2}+\ldots
$$

Remark 20. By the $a d$-nilpotence of $x$ the sum is finite so $\exp \left(a d_{x}\right)$ is well defined.

Remark 21. $\exp \left(a d_{x}\right) \in \operatorname{Aut}(L)$.

Proof. $\exp \left(a d_{x}\right)=1+\eta$, where $\eta$ is a nilpotent summand, therefore it is invertible because $(1+\eta)\left(1-\eta+\eta^{2}-\eta^{3}+\ldots\right)=1$. Let us show that $\exp \left(a d_{x}\right)$ is a homomorphism of Lie algebras. First we will prove by induction on $n \geq 0$ that

$$
\left(a d_{x}\right)^{n}[y, z]=\sum_{i=0}^{n}\left(\begin{array}{l}
n \\
i
\end{array}\right)\left[\left(a d_{x}\right)^{i}(y),\left(a d_{x}\right)^{n-i}(z)\right] .
$$

If $n=0$ there is nothing to prove. For $n+1$,

$$
\begin{array}{r}
\left(a d_{x}\right)^{n+1}[y, z]= \\
\left(a d_{x}\right)^{n}\left(a d_{x}\right)[y, z]= \\
\left(a d_{x}\right)^{n}\left[a d_{x}(y), z\right]+\left(a d_{x}\right)^{n}\left[y, a d_{x}(z)\right]= \\
\sum_{i=0}^{n}\left(\begin{array}{c}
n \\
i
\end{array}\right)\left[\left(a d_{x}\right)^{i+1}(y),\left(a d_{x}\right)^{n-i}(z)\right]+\sum_{i=0}^{n}\left(\begin{array}{c}
n \\
i
\end{array}\right)\left[\left(a d_{x}\right)^{i}(y),\left(a d_{x}\right)^{n-i+1}(z)\right]= \\
\sum_{i=1}^{n+1}\left(\begin{array}{c}
n \\
i-1
\end{array}\right)\left[\left(a d_{x}\right)^{i}(y),\left(a d_{x}\right)^{n-i+1}(z)\right]+\sum_{i=0}^{n}\left(\begin{array}{c}
n \\
i
\end{array}\right)\left[\left(a d_{x}\right)^{i}(y),\left(a d_{x}\right)^{n-i+1}(z)\right]= \\
\sum_{i=0}^{n+1}\left(\begin{array}{c}
n+1 \\
i
\end{array}\right)\left[\left(a d_{x}\right)^{i}(y),\left(a d_{x}\right)^{n+1-i}(z)\right]
\end{array}
$$

where we used that $\left(\begin{array}{c}n+1 \\ i\end{array}\right)=\left(\begin{array}{c}n \\ i-1\end{array}\right)+\left(\begin{array}{c}n \\ i\end{array}\right)$. Rearranging formula (1.17) we have:

$$
\frac{\left(a d_{x}\right)^{n}[y, z]}{n !}=\sum_{i=0}^{n}\left[\frac{\left(a d_{x}\right)^{i}(y)}{i !}, \frac{\left(a d_{x}\right)^{n-i}(z)}{(n-i) !}\right] .
$$


Since $a d_{x}$ is nilpotent, there exists some $k$ such that $\left(a d_{x}\right)^{k}=0$. Suppose that $m$ is the minimum of the set $\left\{k \in \mathbb{Z}_{+} \mid\left(a d_{x}\right)^{k}=0\right\}$, then

$$
\begin{array}{r}
{\left[\exp \left(a d_{x}\right)(y), \exp \left(a d_{x}\right)(z)\right]=} \\
{\left[\sum_{i=0}^{m-1} \frac{\left(a d_{x}\right)^{i}(y)}{i !}, \sum_{j=0}^{m-1} \frac{\left(a d_{x}\right)^{j}(z)}{j !}\right]=} \\
\sum_{i=0}^{m-1} \sum_{j=0}^{m-1}\left[\frac{\left(a d_{x}\right)^{i}(y)}{i !}, \frac{\left(a d_{x}\right)^{j}(z)}{j !}\right]
\end{array}
$$

rearranging in a way that $i+j=n$ for $n=0, \ldots, 2 m-2$ we have

$$
\sum_{n=0}^{2 m-2}\left(\sum_{i=0}^{n}\left[\frac{\left(a d_{x}\right)^{i}(y)}{i !}, \frac{\left(a d_{x}\right)^{n-i}(z)}{(n-i) !}\right]\right)
$$

and by formula (1.18),

$$
\sum_{n=0}^{2 m-2} \frac{\left(a d_{x}\right)^{n}[y, z]}{n !}=\sum_{n=0}^{m-1} \frac{\left(a d_{x}\right)^{n}[y, z]}{n !}=\exp \left(a d_{x}\right)([y, z])
$$

because of the nilpotency of $a d_{x}$.

Remark 22. In the proof of Remark (21) we only used that $a d_{x}$ is a nilpotent derivation.

Remark 23. Let $\phi: L \rightarrow L^{\prime}$ be a homomorphism of Lie algebras such that $x \in L$ is ad-nilpotent and $\phi(x)$ is ad-nilpotent. Then

$$
\exp \left(a d_{\phi(x)}\right) \phi(y)=\phi\left(\exp \left(a d_{x}\right)(y)\right)
$$

Proof. Let us prove by induction on $n$ that

$$
\left(a d_{\phi(x)}\right)^{n}(\phi(y))=\phi\left(\left(a d_{x}\right)^{n}(y)\right)
$$

If $n=0$ there is nothing to prove. Let us prove formula (1.20) for $n+1$. $\left(a d_{\phi(x)}\right)^{n+1}(\phi(y))=\left(a d_{\phi(x)}\right)^{n} a d_{\phi(x)}(\phi(y))=\left(a d_{\phi(x)}\right)^{n}([\phi(x), \phi(y)])=$ $\left(a d_{\phi(x)}\right)^{n}(\phi[x, y])=\left(a d_{\phi(x)}\right)^{n}\left(\phi\left(a d_{x}(y)\right)\right)=\phi\left(\left(a d_{x}\right)^{n}\left(a d_{x}(y)\right)\right)=$ $\phi\left(\left(a d_{x}\right)^{n+1}(y)\right)$ because $\phi$ is a homomorphism of Lie algebras and because of 
induction hypothesis. Since $x$ is ad-nilpotent there exists some $m \in \mathbb{Z}_{+}$ such that $\left(a d_{x}\right)^{m}=0$, therefore $\exp \left(a d_{\phi(x)}\right) \phi(y)=\sum_{i=0}^{\infty} \frac{\left(a d_{\phi(x)}\right)^{i}(\phi(y))}{i !}=$ $\sum_{i=0}^{m-1} \frac{\left(a d_{\phi(x)}\right)^{i}(\phi(y))}{i !}=\sum_{i=0}^{m-1} \frac{\phi\left(\left(a d_{x}\right)^{i}(y)\right)}{i !}=\phi\left(\sum_{i=0}^{m-1} \frac{\left(a d_{x}\right)^{i}(y)}{i !}\right)=\phi\left(\sum_{i=0}^{\infty} \frac{\left(a d_{x}\right)^{i}(y)}{i !}\right)$ $=\phi\left(\exp \left(a d_{x}\right)(y)\right)$ because $\phi$ is a homomorphism of Lie algebras hence $\phi$ is a linear application.

Remark 24. In the definition of $\exp \left(a d_{x}\right)$ we requested the nilpotency of $a d_{x}$. Anyway $\exp \left(a d_{x}\right)$ is well defined even when $a d_{x}$ is not nilpotent but locally nilpotent, i.e. it satisfies the following property: for all $y \in L$ there exists some $m_{y} \in \mathbb{Z}_{+}$such that $\left(a d_{x}\right)^{m_{y}} y=0$.

Let us consider $\mathfrak{s l}_{2}(\mathbb{F})=\langle x, y, h\rangle$ where

$$
x=\left(\begin{array}{ll}
0 & 1 \\
0 & 0
\end{array}\right), y=\left(\begin{array}{ll}
0 & 0 \\
1 & 0
\end{array}\right), h=\left(\begin{array}{cc}
1 & 0 \\
0 & -1
\end{array}\right) .
$$

Since $x, y$ are nilpotent, they are $a d$-nilpotent therefore the maps $\exp \left(a d_{x}\right)$ and $\exp \left(a d_{y}\right)$ are well defined. We set

$$
\sigma=\exp \left(a d_{x}\right) \exp \left(a d_{-y}\right) \exp \left(a d_{x}\right)
$$

Remark 25.

$$
\begin{gathered}
\sigma(h)=-h \\
\sigma(x)=-y \\
\sigma(y)=-x
\end{gathered}
$$

Proof. A brief calculation shows that, with respect to the basis $\{x, y, h\}$ of $\mathfrak{s l}_{2}(\mathbb{F})$ :

$$
\exp \left(a d_{x}\right)=\left(\begin{array}{ccc}
1 & -1 & -2 \\
0 & 1 & 0 \\
0 & 1 & 1
\end{array}\right)
$$

and

$$
\exp \left(a d_{-y}\right)=\left(\begin{array}{ccc}
1 & 0 & 0 \\
-1 & 1 & -2 \\
1 & 0 & 1
\end{array}\right)
$$


hence

$$
\sigma=\left(\begin{array}{ccc}
0 & -1 & 0 \\
-1 & 0 & 0 \\
0 & 0 & -1
\end{array}\right)
$$

i.e. $\sigma(h)=-h, \sigma(x)=-y, \sigma(y)=-x$.

Proposition 1.3.1. Let $x \in L \subseteq \mathfrak{g l}(V)$ be locally nilpotent, and let $y \in L$. Then $\exp (x) y(\exp (x))^{-1}=\exp \left(a d_{x}\right)(y)$.

Proof. Notice that $a d_{x}=\lambda_{x}+\rho_{-x}$ where $\lambda_{x}$ is the left multiplication by $x$ and $\rho_{x}$ is the right multiplication by $x$. $\lambda_{x}$ and $\rho_{-x}$ commute, then $\exp \left(a d_{x}\right)(y)=$ $\exp \left(\lambda_{x}+\rho_{-x}\right)(y)=\exp \left(\lambda_{x}\right) \exp \left(\rho_{-x}\right)(y)=\exp \left(\lambda_{x}\right) \quad \sum_{i=0}^{\infty} \frac{1}{i !}\left(\rho_{-x}\right)^{i}(y)=$ $\exp \left(\lambda_{x}\right) \sum_{i=0}^{\infty} \frac{1}{i !}(-1)^{i} y x^{i}=\exp \left(\lambda_{x}\right)(y \exp (-x))=\exp \left(\lambda_{x}\right)\left(y(\exp (x))^{-1}\right)=$ $\left(\sum_{i=0}^{\infty} \frac{1}{i !} x^{i} y(\exp (x))^{-1}\right)=\exp (x) y(\exp (x))^{-1}$.

Let us consider the map $s=\exp (\phi(x)) \exp (\phi(-y)) \exp (\phi(x))$ where $\phi$ : $\mathfrak{s l}_{2}(\mathbb{F}) \rightarrow \mathfrak{g l}(V)$ is a representation. For $z$ in $\mathfrak{s l}_{2}(\mathbb{F})$ we have

$$
\begin{array}{r}
s \phi(z) s^{-1}=\exp (\phi(x)) \exp (\phi(-y)) \exp (\phi(x)) \phi(z)(\exp (\phi(x)))^{-1} \\
(\exp (\phi(-y)))^{-1}(\exp (\phi(x)))^{-1}
\end{array}
$$

by Proposition (1.3.1) we have:

$$
\begin{gathered}
\left(\exp (\phi(x)) \phi(z)(\exp (\phi(x)))^{-1}=\exp \left(a d_{\phi(x)}\right)(\phi(z))=\phi\left(\exp \left(a d_{x}\right)(z)\right) .\right. \\
\exp (\phi(-y)) \phi\left(\exp \left(a d_{x}\right)(z)\right)(\exp (\phi(-y)))^{-1}= \\
\exp \left(a d_{\phi(-y)}\right)\left(\phi\left(\exp \left(a d_{x}\right)(z)\right)\right)=\phi\left(\exp \left(a d_{-y}\right) \exp \left(a d_{x}\right)(z)\right) \\
\exp (\phi(x)) \phi\left(\exp \left(a d_{-y}\right) \exp \left(a d_{x}\right)(z)\right)(\exp (\phi(x)))^{-1}= \\
\exp \left(a d_{\phi(x)}\right)\left(\phi\left(\exp \left(a d_{-y}\right) \exp \left(a d_{x}\right)(z)\right)\right)= \\
\phi\left(\exp \left(a d_{x}\right) \exp \left(a d_{-y}\right) \exp \left(a d_{x}\right)(z)\right)
\end{gathered}
$$

Hence

$$
s \phi(z) s^{-1}=\phi\left(\exp \left(a d_{x}\right) \exp \left(a d_{-y}\right) \exp \left(a d_{x}\right)(z)\right)=\phi(\sigma(z)) .
$$

Summarizing: 
Proposition 1.3.2. Let $\phi$ be a representation of $\mathfrak{s l}_{2}(\mathbb{F})$ on a vector space $V$, let $z$ be in $\mathfrak{s l}_{2}(\mathbb{F})$ and let $s=\exp (\phi(x)) \exp (\phi(-y)) \exp (\phi(x))$ and $\sigma=$ $\exp \left(a d_{x}\right) \exp \left(a d_{-y}\right) \exp \left(a d_{x}\right)$. Then

$$
s \phi(z) s^{-1}=\phi(\sigma(z)) .
$$

Remark 26. $s \phi(h) s^{-1}=\phi(\sigma(h))=-\phi(h)$.

Let, now, $L$ be a semisimple Lie algebra, let $\alpha$ be a root in $\Phi^{+}$and let $\left\langle x_{\alpha}, y_{\alpha},\left[x_{\alpha}, y_{\alpha}\right]=h_{\alpha}\right\rangle=\mathfrak{s l}_{2}(\alpha) \cong \mathfrak{s l}_{2}(\mathbb{F})$ be a copy of $\mathfrak{s l}_{2}(\mathbb{F})$ into $L$. Let us denote, for brevity $x_{\alpha}=x, y_{\alpha}=y, h_{\alpha}=h$. Then the following proposition holds:

Proposition 1.3.3. Let $k \in H$. Then $s \phi(k) s^{-1}=\phi(k-\alpha(k) h)$

Proof. We already know, by Proposition (1.3.2), that $s \phi(k) s^{-1}=\phi(\sigma(k))$. If $k=h$, there is nothing new and we know that $s \phi(h) s^{-1}=\phi(\sigma(h))=-\phi(h)$. As for the right hand side, $\phi(h-\alpha(h) h)=-\phi(h)$ because $\alpha(h)=2$. The same thing holds for $k=\lambda h$, by linearity. So let us now suppose that $k$ and $h$ are linearly independent and let $N=\langle x, y, h, k\rangle$. Let us compute the matrices associated to $\exp \left(a d_{x}\right)$ and $\exp \left(a d_{-y}\right)$ with respect to the basis $\{x, y, h, k\}$.

$$
a d_{x}=\left(\begin{array}{cccc}
0 & 0 & -2 & -\alpha(k) \\
0 & 0 & 0 & 0 \\
0 & 1 & 0 & 0 \\
0 & 0 & 0 & 0
\end{array}\right),\left(a d_{x}\right)^{2}=\left(\begin{array}{cccc}
0 & -2 & 0 & 0 \\
0 & 0 & 0 & 0 \\
0 & 0 & 0 & 0 \\
0 & 0 & 0 & 0
\end{array}\right)
$$

because $a d_{x}(k)=[x, k]=-\alpha(k) x$. Similarly,

$$
a d_{-y}=\left(\begin{array}{cccc}
0 & 0 & 0 & 0 \\
0 & 0 & -2 & -\alpha(k) \\
1 & 0 & 0 & 0 \\
0 & 0 & 0 & 0
\end{array}\right),\left(a d_{-y}\right)^{2}=\left(\begin{array}{cccc}
0 & 0 & 0 & 0 \\
-2 & 0 & 0 & 0 \\
0 & 0 & 0 & 0 \\
0 & 0 & 0 & 0
\end{array}\right) .
$$


Therefore

$\exp \left(a d_{x}\right)=\left(\begin{array}{cccc}1 & -1 & -2 & -\alpha(k) \\ 0 & 1 & 0 & 0 \\ 0 & 1 & 1 & 0 \\ 0 & 0 & 0 & 1\end{array}\right), \exp \left(a d_{-y}\right)=\left(\begin{array}{cccc}1 & 0 & 0 & 0 \\ -1 & 1 & -2 & -\alpha(k) \\ 1 & 0 & 1 & 0 \\ 0 & 0 & 0 & 1\end{array}\right)$

and

$$
\begin{array}{r}
s \phi(k) s^{-1}=\phi(\sigma(k))=\phi\left(\left(\begin{array}{cccc}
0 & -1 & -1-\alpha(k) & 0 \\
-1 & 0 & 0 & 0 \\
0 & 0 & -1 & -\alpha(k) \\
0 & 0 & 0 & 1
\end{array}\right)\left(\begin{array}{l}
0 \\
0 \\
0 \\
1
\end{array}\right)\right) \\
=\phi\left(\left(\begin{array}{c}
0 \\
0 \\
-\alpha(k) \\
1
\end{array}\right)\right)=\phi(k-\alpha(k) h)
\end{array}
$$




\section{Chapter 2}

\section{Weyl's Theorem}

\subsection{The Casimir element}

Let $L$ be a semisimple Lie algebra of finite dimension, let $\phi: L \rightarrow \mathfrak{g l}(V)$ be a faithful representation of $L$ and let $\beta: L \times L \rightarrow F$ be the following bilinear form on $L$ :

$$
\beta(x, y)=\operatorname{tr}(\phi(x) \phi(y))
$$

Remark 27. $\beta$ is associative, i.e. $\beta([x, y], z)=\beta(x,[y, z])$

Proof. $\beta([x, y], z)=\operatorname{tr}(\phi([x, y]) \phi(z))=\operatorname{tr}([\phi(x), \phi(y)] \phi(z))=$ $\operatorname{tr}(\phi(x) \phi(y) \phi(z)-\phi(y) \phi(x) \phi(z))=\operatorname{tr}(\phi(x) \phi(y) \phi(z)-\phi(z) \phi(y) \phi(x))=$ $\operatorname{tr}(\phi(x)[\phi(y), \phi(z)])=\beta(x,[y, z])$ where we used the linearity of the trace and its property $\operatorname{tr}(a b)=\operatorname{tr}(b a)$.

Remark 28. The radical of the form, i.e. the set $\operatorname{Rad} \beta=\{x \in L \mid \beta(x, y)=$ 0 for all $y \in L\}$, is an ideal of $L$.

Proof. Let $x$ be in $\operatorname{Rad} \beta$. Then, given $z, y \in L, \beta([x, z], y)=\beta(x,[z, y])=0$ since $[z, y]$ in $L$ and $\beta$ is associative.

Remark 29. $\operatorname{Rad} \beta$ is solvable. 
Proof. $\phi$ is a faithful representation of $L$ so $\phi(\operatorname{Rad} \beta) \cong \operatorname{Rad} \beta$. For every $x, y \in \operatorname{Rad} \beta, 0=\beta(x, y)=\operatorname{tr}(\phi(x) \phi(y))$ therefore, as $\phi(\operatorname{Rad} \beta) \leq \mathfrak{g l}(V)$, by Cartan's Criterion, $\phi(\operatorname{Rad} \beta)$ is solvable hence $\operatorname{Rad} \beta$ is solvable.

Thanks to the Remarks (28) and (29) and to the fact that $L$ is semisimple, $\operatorname{Rad} \beta=0$, i.e. $\beta$ is nondegenerate. If $\left\{x_{1}, \ldots, x_{n}\right\}$ is a basis of $L$, then there is a unique determined basis $\left\{y_{1}, \ldots, y_{n}\right\}$ of $L$ such that $\beta\left(x_{i}, y_{j}\right)=\delta_{i j}$ (dual basis).

Definition 2.1. The Casimir element is defined as the following endomorphism of $\operatorname{End}(V)$ :

$$
C_{\phi}=\sum_{i=1}^{n} \phi\left(x_{i}\right) \phi\left(y_{i}\right)
$$

where $\left\{x_{1}, \ldots, x_{n}\right\}$ and $\left\{y_{1}, \ldots, y_{n}\right\}$ are dual basis defined as above.

Remark 30. $C_{\phi}$ is a homomorphism of $L$-modules.

Proof. Let $\left[x, x_{i}\right]=\sum_{j=1}^{n} a_{i j} x_{j}$ and let $\left[x, y_{i}\right]=\sum_{j=1}^{n} b_{i j} y_{j} . \quad \beta\left(\left[x, x_{i}\right], y_{k}\right)=$ $\sum_{j=1}^{n} a_{i j} \beta\left(x_{j}, y_{k}\right)=\sum_{j=1}^{n} a_{i j} \delta_{j k}=a_{i k}$. On the other hand $\beta\left(\left[x, x_{i}\right], y_{k}\right)=$ $-\beta\left(\left[x_{i}, x\right], y_{k}\right)=-\beta\left(x_{i},\left[x, y_{k}\right]\right)=-\sum_{j=1}^{n} b_{k j} \beta\left(x_{i}, y_{j}\right)=\sum_{j=1}^{n} b_{k j} \delta_{i j}=-b_{k i}$. Therefore $a_{i k}=-b_{k i}$. Now let us consider $\left[\phi(x), C_{\phi}\right]$. [ $\left.\phi(x), C_{\phi}\right]=\sum_{i=1}^{n}[\phi(x)$, $\left.\phi\left(x_{i}\right) \phi\left(y_{i}\right)\right]=\sum_{i=1}^{n}\left(\phi(x) \phi\left(x_{i}\right) \phi\left(y_{i}\right)-\phi\left(x_{i}\right) \phi\left(y_{i}\right) \phi(x)\right)=\sum_{i=1}^{n}\left(\phi(x) \phi\left(x_{i}\right) \phi\left(y_{i}\right)-\right.$ $\left.\phi\left(x_{i}\right) \phi(x) \phi\left(y_{i}\right)+\phi\left(x_{i}\right) \phi(x) \phi\left(y_{i}\right)-\phi\left(x_{i}\right) \phi\left(y_{i}\right) \phi(x)\right)=\sum_{i=1}^{n}\left(\left[\phi(x), \phi\left(x_{i}\right)\right] \phi\left(y_{i}\right)+\right.$ $\left.\phi\left(x_{i}\right)\left[\phi(x), \phi\left(y_{i}\right)\right]\right)=\sum_{i=1}^{n}\left(\phi\left(\left[x, x_{i}\right]\right) \phi\left(y_{i}\right)+\phi\left(x_{i}\right) \phi\left(\left[x, y_{i}\right]\right)\right)=\sum_{i, k=1}^{n}\left(a_{i k} \phi\left(x_{k}\right)\right.$ $\left.\phi\left(y_{i}\right)+b_{i k} \phi\left(x_{i}\right) \phi\left(y_{k}\right)\right)=0$ because $a_{i k}=-b_{k i}$. Thus $0=\left[\phi(x), C_{\phi}\right]=$ $\phi(x) C_{\phi}-C_{\phi} \phi(x)$ that is $C_{\phi}$ is a homomorphism of $L$-modules.

Lemma 2.1.1 (Schur's Lemma). Let $\mathbb{F}$ be an algebraically closed field and let $V$ be an irreducible L-module. If $\varphi$ is an endomorphism of $V$ which is also a homomorphism of $L$-modules with $L$ a Lie algebra, then $\varphi$ is $\lambda$ id with $\lambda$ in $\mathbb{F}$.

Proof. Let $\lambda$ be an eigenvalue of $\varphi$ and $V_{\lambda}$ be an eigenspace. Then $V_{\lambda}$ is a $L$-submodule of $V$, indeed, if $v$ in $V_{\lambda}$ then $\varphi(x . v)=x . \varphi(v)=\lambda x . v$, hence, by the irreducibility of $V, V=V_{\lambda}$. 
Remark 31. By Schur's Lemma, if $\mathbb{F}$ is an algebraically closed field and $\phi$ : $L \rightarrow \mathfrak{g l}(V)$ is an irreducible faithful representation, then $C_{\phi}$ acts as a scalar. Moreover $\operatorname{tr}\left(C_{\phi}\right)=\operatorname{tr}\left(\sum_{i=1}^{n} \phi\left(x_{i}\right) \phi\left(y_{i}\right)\right)=\sum_{i=1}^{n} \beta\left(x_{i}, y_{i}\right)=\sum_{i=1}^{n} 1=\operatorname{dim} L$.

\section{$2.2 \quad$ Weyl's Theorem}

Lemma 2.2.1. Let $L$ be a semisimple Lie algebra and let $\varphi: L \rightarrow \mathfrak{g l}(V)$ be a representation of $L$ in $V$ with $\operatorname{dim} V=1$. Then $\varphi=0$.

Proof. Since $L$ is a semisimple Lie algebra, we have $L=[L, L]$. It follows that every $x \in L$ can be written in the form $x=\sum_{i}\left[y_{i}, z_{i}\right], \varphi(x)=\varphi\left(\sum_{i}\left[y_{i}, z_{i}\right]\right)=$ $\sum_{i}\left[\varphi\left(y_{i}\right), \varphi\left(z_{i}\right)\right]=\sum_{i}\left(\varphi\left(y_{i}\right) \varphi\left(z_{i}\right)-\varphi\left(z_{i}\right) \varphi\left(y_{i}\right)\right)=0$ because, if $0 \neq v \in V$, then $\varphi\left(z_{i}\right)(\lambda v)=\lambda \mu_{z_{i}} v$ for a proper $\mu_{z_{i}} \in \mathbb{F}$ because $\operatorname{dim} V=1$, therefore $\varphi\left(y_{i}\right) \varphi\left(z_{i}\right)(\lambda v)=\lambda \mu_{y_{i}} \mu_{z_{i}} v=\lambda \mu_{z_{i}} \mu_{y_{i}} v=\varphi\left(z_{i}\right) \varphi\left(y_{i}\right)(\lambda v)$, that is $\varphi\left(y_{i}\right) \varphi\left(z_{i}\right)=$ $\varphi\left(z_{i}\right) \varphi\left(y_{i}\right)$.

Lemma 2.2.2. Let $\phi: L \rightarrow \mathfrak{g l}(V)$ be a finite dimensional representation of a semisimple Lie algebra $L$ and let $W$ be an irreducible submodule of codimension 1 in $V$. Then there exists an $L$-submodule $U$ such that $V=$ $W \oplus U$.

Proof. We can assume that $\phi$ is a faithful representation because if $\phi$ is not so, since $L=\oplus_{i} L_{i}$ with $L_{i}$ simple Lie algebras, $L / \operatorname{Ker} \phi=\oplus_{i} L_{i} / \operatorname{Ker} \phi$, i.e. $L / \operatorname{Ker} \phi$ is semisimple and the homomorphism $\tilde{\phi}$ induced by $\phi$ on $L / \operatorname{Ker} \phi$ is injective. Let us consider a basis $\left\{w_{1}, \ldots, w_{n-1}\right\}$ of $W$ and let us complete $\left\{w_{1}, \ldots, w_{n-1}\right\}$ to a basis of $V,\left\{w_{1}, \ldots, w_{n-1}, v\right\}$. Since $V / W$ is an $L$-module of dimension 1, by Lemma (2.2.1), $\phi$ acts in a trivially way on $V / W$, i.e. $\phi(x) v$ is in $W$ for every $v$ in $V$. Therefore the restriction of $C_{\phi}$ to $W$ is in $\operatorname{End}(W)$. $C_{\phi}$ is a homomorphism of $L$-modules, $C_{\phi}$ restricted to $W$ is an endomorphism of $W, W$ is an irreducible $L$-module hence, by Schur's Lemma, $C_{\phi}$ acts as a scalar, $\lambda$, on $W$. Thus the matrix associated to $C_{\phi}$, with respect to the basis 
$\left\{w_{1}, \ldots, w_{n-1}, v\right\}$, is

$$
\left(\begin{array}{llll}
\lambda & & & * \\
& \ddots & & * \\
& & \lambda & * \\
0 & 0 & 0 & 0
\end{array}\right) .
$$

$\operatorname{Rank} C_{\phi}=n-1=\operatorname{dim} W$ because $\lambda(n-1)=\operatorname{tr}\left(C_{\phi}\right)=\operatorname{dim} L$ which means that $\lambda$ is not 0 . It follows that $\operatorname{dim} V=\operatorname{dim} \operatorname{Ker} C_{\phi}+\operatorname{Rank} C_{\phi}=$ $\operatorname{dim} \operatorname{Ker} C_{\phi}+\operatorname{dim} W$. Moreover $\operatorname{Ker} C_{\phi} \cap W=0$ because $C_{\phi}$ acts on $W$ as the scalar $\lambda \neq 0$ and $\operatorname{Ker} C_{\phi}$ is an $L$-submodule of $V$. For these reasons $V=W \oplus \operatorname{Ker} C_{\phi}$.

Lemma 2.2.3. Let $V$ be a finite dimensional $L$-module, with $L$ a semisimple Lie algebra, let $W$ be an L-submodule of codimension 1 in $V$. Then there exists an L-module, $U$, such that $V=W \oplus U$.

Proof. Let us prove this lemma by induction on $\operatorname{dim} W$. If $\operatorname{dim} W=1, W$ is irreducible then the lemma is proved for Lemma (2.2.2). So let $\operatorname{dim} W>1$. If $W$ is irreducible, then the lemma is proved, therefore let us suppose $W$ non irreducible. As $W$ is not irreducible, there exists a proper $L$-submodule of $W, W^{\prime}$, and $W / W^{\prime}$ is an $L$-module of codimension 1 in $V / W^{\prime}$ with lesser dimension. Using induction there exists an $L$-submodule $\tilde{W}$ such that $V / W^{\prime}=W / W^{\prime} \oplus \tilde{W} / W^{\prime}$ and $\operatorname{dim} \tilde{W} / W^{\prime}=1$, that is $W^{\prime}$ has codimension 1 in $\tilde{W}$. Since $\operatorname{dim} W^{\prime}<\operatorname{dim} W$, using for a second time induction there exists an $L$-submodule, $X$, of $W^{\prime}$ such that $\tilde{W}=W^{\prime} \oplus X$. But $X \cap W=0$, because $X \subseteq \tilde{W}$ and $\tilde{W} \cap W=W^{\prime}$. Hence $X$ is an $L$-submodule of $V$ such that $V=W \oplus X$.

Theorem 2.2.4 (Weyl's Theorem). Let $V$ be a finite dimensional L-module. Then $V$ is completely reducible.

Proof. Let us prove that, for every $L$-submodule $W$ of $V$, there exists another $L$-submodule, $W^{\prime}$, such that $V=W \oplus W^{\prime}$. Let us consider $A=\{\varphi \in$ $\left.\operatorname{Hom}(V, W)|\varphi|_{W}=0\right\}$ and $B=\left\{\varphi \in \operatorname{Hom}(V, W)|\varphi|_{W}=a i d, a \in \mathbb{F}\right\}$. 
Since $V, W$ are $L$-modules, $\operatorname{Hom}(V, W)$ is also an $L$-module with the action of $L$ on $\operatorname{Hom}(V, W)$ defined as follows: $(x . \varphi)(v)=-\varphi(x . v)+x \cdot(\varphi(v))$. If $\varphi$ is such that $\left.\varphi\right|_{W}=a i d,(x . \varphi)(w)=-a(x . w)+x . a w=0$ hence $A, B$ are $L$-modules. Moreover the action of $L$ sends $B$ to $A$. Let us now consider the projection $\pi$ of $V$ to $W$. Notice that $\pi$ lies in $B$, but not in $A$. An element $\varphi$ in $B$ can be written as follows: $\varphi=(\varphi-a \pi)+a \pi$. The first summand is an element of $A$ and the second summand is an element of $B$, so $A$ has codimension 1 in $B$ and, thanks to Lemma (2.2.3) there exists an $L$-module $C$ such that $B=A \oplus C$. Let $\varphi \neq 0$ be in $C$. Then, by Lemma (2.2.1), $x . \varphi=0$ because $L$ is a semisimple Lie algebra and $\operatorname{dim} C=1$. Hence $\varphi$ is a homomorphism of $L$-modules because, for every $x$ in $L$ and $v$ in $V, x \cdot(\varphi(v))=\varphi(x . v)$. It follows that $\operatorname{Ker} \varphi$ is an $L$-module besides, $\operatorname{Ker} \varphi \cap W=0$, because $\varphi$ is in $C$ and $\varphi \neq 0$, so its restriction to $W$ is not 0 , and $\operatorname{dim} W+\operatorname{dim} \operatorname{Ker} \varphi=\operatorname{dim} \operatorname{Im} \varphi+\operatorname{dim} \operatorname{Ker} \varphi=\operatorname{dim} V$. Thus $V=W \oplus \operatorname{Ker} \varphi$. 


\section{Chapter 3}

\section{Finite dimensional}

\section{representations of $\mathfrak{s l}_{2}(\mathbb{F})$}

In this chapter $\mathbb{F}$ is an algebraically closed field with characteristic 0 and $V \neq 0$ is a finite dimensional $\mathfrak{s l}_{2}(\mathbb{F})$-module.

Let us consider a basis of $\mathfrak{s l}_{2}(\mathbb{F})$ given by $\{x, y, h\}$ where

$$
x=\left(\begin{array}{ll}
0 & 1 \\
0 & 0
\end{array}\right), y=\left(\begin{array}{ll}
0 & 0 \\
1 & 0
\end{array}\right), h=\left(\begin{array}{cc}
1 & 0 \\
0 & -1
\end{array}\right)
$$

then $[x, y]=h,[h, x]=2 x,[h, y]=-2 y$. Since $h$ is semisimple and $\mathbb{F}$ is an algebraically closed field, $h$ acts diagonally on $V$ and $V$ admits a decomposition as direct sum of eigenspaces $V_{\lambda}=\{v \in V \mid h . v=\lambda v\}$. When $V_{\lambda} \neq 0$ we call it a weight space and $\lambda$ a weight.

Lemma 3.0.5. Let $v$ be in $V_{\lambda}$. Then $x . v$ lies in $V_{\lambda+2}$ and $y . v$ lies in $V_{\lambda-2}$.

Proof. h. $(x . v)=[h, x] . v+x .(h . v)=2 x . v+\lambda x . v=(\lambda+2) x . v$.

$h \cdot(y \cdot v)=[h, y] \cdot v+y \cdot(h \cdot v)=-2 y \cdot v+\lambda y \cdot v=(\lambda-2) y \cdot v$.

Since $V$ is finite dimensional and eigenvectors corresponding to different eigenvalues are linearly independent, there exists some $\lambda$ such that $V_{\lambda} \neq 0$ and $V_{\lambda+2}=0$, i.e. for every $v \in V_{\lambda}, x . v=0$. Any nonzero vector of $V_{\lambda}$, for $\lambda$ as taken above, is called a maximal vector of weight $\lambda$. 


\subsection{Irreducible finite dimensional representa- tions}

Let us consider an irreducible finite dimensional $\mathfrak{s l}_{2}(\mathbb{F})$-module, $V$, and a maximal vector $v_{0}$ in $V$. If we set $v_{i}=y \cdot v_{i-1}$ for all $i>0$, there exists some $m \in \mathbb{Z}_{+}$such that $v_{m} \neq 0$ and $v_{m+1}=0$, because $V$ is finite dimensional.

Theorem 3.1.1. $\left\{v_{0}, \ldots, v_{m}\right\}$ is a basis of $V$.

Proof. $v_{0}, \ldots, v_{m}$ are linearly independent because they are eigenvectors with different eigenvalues so if $V^{\prime}=\left\langle v_{0}, \ldots, v_{m}\right\rangle$ is a $\mathfrak{s l}_{2}(\mathbb{F})$-module, then by the irreducibility of $V,\left\langle v_{0}, \ldots, v_{m}\right\rangle=V$. The action of $y$ and $h$ stabilizes $V^{\prime}$ by construction. Let us prove by induction on $i$ that $x \cdot v_{i}=i(\lambda-i+1) v_{i-1}$. $x \cdot v_{0}=0$ for the choice of $v_{0}, x \cdot v_{i+1}=x \cdot\left(y \cdot v_{i}\right)=[x, y] \cdot v_{i}+y \cdot\left(x \cdot v_{i}\right)=h \cdot v_{i}+$ $i(\lambda-i+1) y \cdot v_{i-1}=(\lambda-2 i) v_{i}+i(\lambda-i+1) v_{i}=(i+1)(\lambda-i) v_{i}$. Hence the action of $x$ stabilizes $V^{\prime}$ too.

Remark 32. Every weight space $V_{\mu}$ has dimension 1 because $v_{0}, \ldots, v_{m}$ are eigenvectors with distinct eigenvalues.

Remark 33. We can apply the calculus made above to the case $i=m+1$. In this case we obtain: $0=x \cdot v_{m+1}=(m+1)(\lambda-m) v_{m}$. Since $m+1 \neq 0$ (it is the dimension of $V \neq 0), v_{m} \neq 0$ by hypothesis and the field $\mathbb{F}$ has characteristic 0 , it follows that $\lambda=m$.

Summarizing:

Theorem 3.1.2. Let $V$ be an irreducible finite dimensional $\mathfrak{s l}_{2}(\mathbb{F})$-module with $\mathbb{F}$ algebraically closed field with characteristic 0 . Then

1. $V$ is the direct sum of weight spaces $V_{\mu}$ relative to $h$ with $\mu=m, m-$ $2, \ldots,-m+2,-m$, where $m=\operatorname{dim} V-1$ and $\operatorname{dim} V_{\mu}=1$ for each $\mu$.

2. $V$ has, up to nonzero scalar multiples, a unique maximal vector of weight $m$. 
3. The above formulas describe completely the action of $\mathfrak{s l}_{2}(\mathbb{F})$ on $V$. In particular there exists at most one irreducible $\mathfrak{s l}_{2}(\mathbb{F})$-module of dimension $m+1$, for every $m \in \mathbb{Z}_{+}$.

Corollary 3.1.3. Let $V$ be a finite dimensional $\mathfrak{s l}_{2}(\mathbb{F})$-module. Then the eigenvalues of $h$ on $V$ are all integers and each occurs along with its negative an equal number of times. Moreover, in any decomposition of $V$ into direct sum of irreducible submodules, the number of summands is dim $V_{0}+\operatorname{dim} V_{1}$.

Proof. If $V=0$ there is nothing to prove. If $V \neq 0$, thanks to Weyl's Theorem, it is possible to write $V$ as a sum of irreducible submodules: $V=$ $\bigoplus V_{i}$ with $V_{i}$ irreducible. For each $V_{i}$, by Theorem (3.1.2), all the eigenvalues are integers and if $l$ is an eigenvalue, $-l$ is also an eigenvalue. Moreover if $\operatorname{dim} V_{i}$ is even, 1 is an eigenvalue and $\operatorname{dim}\left(V_{i}\right)_{1}=1$, if $\operatorname{dim} V_{i}$ is odd, 0 is an eigenvalue and $\operatorname{dim}\left(V_{i}\right)_{0}=1$, then the number of irreducible submodules $V_{i}$ is $\operatorname{dim} V_{0}+\operatorname{dim} V_{1}$.

We have just seen that for every $m \in \mathbb{Z}_{+}$there exists at most one irreducible $\mathfrak{s l}_{2}$-module of dimension $m+1$ which, from now on, we shall denote by $V(m)$. Is there an irreducible $\mathfrak{s l}_{2}(\mathbb{F})$-module of dimension $(m+1)$ for all $m \in \mathbb{Z}_{+}$? For $m=0$ there is the trivial representation, for $m=1$ the standard representation, for $m=2$ the adjoint representation and for the other $m$ it is possible to take a $(m+1)$-dimensional vector space with basis $\left\{v_{0}, \ldots, v_{m}\right\}$ and define the action of $\mathfrak{s l}_{2}(\mathbb{F})$ on this vector space in this way:

$$
\begin{array}{r}
y \cdot v_{i}=v_{i+1} \\
x . v_{i}=i(m-i+1) v_{i-1} \\
h . v_{i}=(m-2 i) v_{i}
\end{array}
$$

with $v_{m+1}=0$. This is clearly an irreducible module of dimension $m+1$ for what we have proved in Theorem (3.1.1).

Equivalently, we can construct the irreducible $\mathfrak{s l}_{2}(\mathbb{F})$-modules explicitly as follows. For every $m \in \mathbb{Z}_{+}$let $V_{m}$ be the vector space of homogeneous 
monomials of degree $m$ in two symbols $X, Y$ with coefficients in $\mathbb{F}$. Of course $\operatorname{dim} V_{m}=m+1$. The linear map

$$
\varphi: \mathfrak{s l}_{2}(\mathbb{F}) \rightarrow\left\langle X \frac{\partial}{\partial Y}, Y \frac{\partial}{\partial X}, X \frac{\partial}{\partial X}-Y \frac{\partial}{\partial Y}\right\rangle
$$

where

$$
\begin{aligned}
\varphi(x) & =X \frac{\partial}{\partial Y} \\
\varphi(y) & =Y \frac{\partial}{\partial X} \\
\varphi(h)=X \frac{\partial}{\partial X} & -Y \frac{\partial}{\partial Y}
\end{aligned}
$$

is an isomorphism of Lie algebras. Indeed it is an isomorphism of vector spaces and is a homomorphism of Lie algebras since $[\varphi(x), \varphi(y)]=\varphi(x) \varphi(y)-$ $\varphi(y) \varphi(x)=\left(X \frac{\partial}{\partial Y}\right)\left(Y \frac{\partial}{\partial X}\right)-\left(Y \frac{\partial}{\partial X}\right)\left(X \frac{\partial}{\partial Y}\right)=X \frac{\partial}{\partial X}-Y \frac{\partial}{\partial Y}=\varphi(h)=\varphi([x, y])$, $[\varphi(h), \varphi(x)]=2 X \frac{\partial}{\partial Y}=2 \varphi(x)=\varphi([h, x])$ and $[\varphi(h), \varphi(y)]=-2 Y \frac{\partial}{\partial X}=$ $-2 \varphi(y)=\varphi([h, y])$.

We now define the following action of $\mathfrak{s l}_{2}(F)$ on $V_{m}$ :

$$
\begin{array}{r}
x \cdot\left(X^{i} Y^{m-i}\right)=X \frac{\partial}{\partial Y}\left(X^{i} Y^{m-i}\right)=(m-i) X^{i+1} Y^{m-i-1} \\
y \cdot\left(X^{i} Y^{m-i}\right)=Y \frac{\partial}{\partial X}\left(X^{i} Y^{m-i}\right)=i X^{i-1} Y^{m-i+1} \\
h .\left(X^{i} Y^{m-i}\right)=\left(X \frac{\partial}{\partial X}-Y \frac{\partial}{\partial Y}\right)\left(X^{i} Y^{m-i}\right)=i X^{i} Y^{m-i}-(m-i) X^{i} Y^{m-i}= \\
(2 i-m) X^{i} Y^{m-i} .
\end{array}
$$

It follows that $X^{m}$ is a maximal vector of weight $m$ hence it generates an irreducible submodule of dimension $m+1$, i.e. the whole $V_{m}$. 


\section{Chapter 4}

\section{Universal enveloping algebras}

The aim of this chapter is to define the universal enveloping algebra $\mathfrak{U}$ of a Lie algebra $L$ and to prove some of its basic properties. Our principal aim will be to use the representation theory of the associative algebra $\mathfrak{U}$ in order to study the representations of $L$. In this chapter $\mathbb{F}$ may be an arbitrary field and $L$ a Lie algebra on $\mathbb{F}$.

\subsection{Definition and some basic properties}

Definition 4.1. A universal enveloping algebra of a Lie algebra $L$ is a pair $(\mathfrak{U}, i)$, where:

1. $\mathfrak{U}$ is an associative algebra with 1 over a field $\mathbb{F}$,

2. $i: L \rightarrow \mathfrak{U}$ is a linear map satisfying

$$
i([x, y])=i(x) i(y)-i(y) i(x)
$$

where $x, y \in L$,

3. for any associative $\mathbb{F}$-algebra $\mathfrak{A}$ with 1 and any linear map $j: L \rightarrow \mathfrak{A}$ satisfying $j([x, y])=j(x) j(y)-j(y) j(x)$, there exists a unique homomorphism of associative algebras $\phi: \mathfrak{U} \rightarrow \mathfrak{A}$ such that $\phi \circ i=j$. 
Remark 34. Denoting with $\mathfrak{U}_{L}$ the structure of Lie algebra on $\mathfrak{U}$, the map $i: L \rightarrow \mathfrak{U}_{L}$ is a homomorphism of Lie algebras.

Theorem 4.1.1. 1. The universal enveloping algebra is unique up to isomorphism.

2. $\mathfrak{U}$ is generated by the image $i(L)$.

3. Let $L_{1}, L_{2}$ be Lie algebras with universal enveloping algebras $\left(\mathfrak{U}_{1}, i_{1}\right),\left(\mathfrak{U}_{2}, i_{2}\right)$ and let $f: L_{1} \rightarrow L_{2}$ be a homomorphism of Lie algebras. Then there exists a homomorphism of algebras $f^{\prime}: \mathfrak{U}_{1} \rightarrow \mathfrak{U}_{2}$ such that $i_{2} \circ f=f^{\prime} \circ i_{1}$.

Proof. 1) Let $(\mathfrak{U}, i)$ and $(\mathfrak{B}, \mathfrak{j})$ be universal enveloping algebras for the Lie algebra $L$. Then, by the defining property (3), there exist a unique homomorphism $j^{\prime}: \mathfrak{U} \rightarrow \mathfrak{B}$ such that $j^{\prime} \circ i=j$ and a unique homomorphism $i^{\prime}: \mathfrak{B} \rightarrow \mathfrak{U}$ such that $i^{\prime} \circ j=i$ therefore $i^{\prime} \circ j^{\prime}$ is a homomorphism of $\mathfrak{U}$ into $\mathfrak{U}$ such that $\left(i^{\prime} \circ j^{\prime}\right) \circ i=i$. Now note that $1_{\mathfrak{U}}$ has the same properties and it is obviously a homomorphism, hence, by the defining property $(3), i^{\prime} \circ j^{\prime}=1_{\mathfrak{U}}$. Similarly $j^{\prime} \circ i^{\prime}=1_{\mathfrak{B}}$. It follows that $j^{\prime}$ is an isomorphism.

2 ) Let $\mathfrak{B}$ be the subalgebra of $\mathfrak{U}$ generated by $i(L)$. Then $i$ can be considered as a homomorphism of $L$ into $\mathfrak{B}_{L}$ so there exists an associative algebras homomorphism $i^{\prime}: \mathfrak{U} \rightarrow \mathfrak{B}$ such that $i^{\prime} \circ i=i$. $i^{\prime}$ can also be considered as a mapping from $\mathfrak{U}$ into $\mathfrak{U} ; 1_{\mathfrak{U}}$ has the same properties and by the uniqueness in the definition $i^{\prime}=1_{\mathfrak{U}}$. Hence $\mathfrak{U}=1_{\mathfrak{U}}(\mathfrak{U})=i^{\prime}(\mathfrak{U}) \subseteq \mathfrak{B}$, but $\mathfrak{B}$ is a subalgebra of $\mathfrak{U}$ so $\mathfrak{U}=\mathfrak{B}$.

$3)$ From the defining property $(2), i_{2}([x, y])=i_{2}(x) i_{2}(y)-i_{2}(y) i_{2}(x)$, therefore the composition $i_{2} \circ f$ is such that $\left(i_{2} \circ f\right)([x, y])=i_{2}(f([x, y]))=$ $i_{2}([f(x), f(y)])=i_{2}(f(x)) i_{2}(f(y))-i_{2}(f(y)) i_{2}(f(x))=\left(i_{2} \circ f\right)(x)\left(i_{2} \circ f\right)(y)-$ $\left(i_{2} \circ f\right)(y)\left(i_{2} \circ f\right)(x)$. Hence, by definition, there exists a unique homomorphism of associative algebras, which we can call $f^{\prime}$, of $\mathfrak{U}_{1}$ into $\mathfrak{U}_{1}$ such that $f^{\prime} \circ i_{1}=i_{2} \circ f$.

Let us now show the existence of the universal enveloping algebra for a Lie algebra $L$. 
Let $\mathfrak{T}(L)$ be the tensor algebra on $L$, i.e.

$$
\mathfrak{T}(L)=\bigoplus_{i=0}^{\infty} T^{i}(L)
$$

where $T^{0}(L)=\mathbb{F}$ and $T^{i}(L)=L \otimes \ldots \otimes L(i$ copies $)$. The vector space operations are the usual ones and the associative product is indicated by " $\otimes$ " and is defined on homogeneous generators of $\mathfrak{T}(L)$ by the rule $\left(x_{1} \otimes\right.$ $\left.\ldots \otimes x_{m}\right) \otimes\left(y_{1} \otimes \ldots \otimes y_{n}\right)=x_{1} \otimes \ldots \otimes x_{m} \otimes y_{1} \otimes \ldots \otimes y_{n} \in T^{m+n}(L) . \mathfrak{T}(L)$ is an associative graded algebra with 1 and has the following universal property: if $\phi: L \rightarrow \mathfrak{A}$ is a $\mathbb{F}$-linear map, where $\mathfrak{A}$ is an associative algebra with 1 , there exists a unique homomorphism of algebras $\psi: \mathfrak{T}(L) \rightarrow \mathfrak{A}$ such that $\psi(1)=1$ and $\psi$ extends the inclusion of $L$ into $\mathfrak{T}(L)$, i.e., calling $i: L \rightarrow \mathfrak{T}(L)$ the inclusion of $L$ into $\mathfrak{T}(L)$, there exists a unique homomorphism of algebras $\psi: \mathfrak{T}(L) \rightarrow \mathfrak{A}$ such that $\psi(1)=1$ and $\psi \circ i=\phi$. Let now $J$ be the two sided ideal in $\mathfrak{T}(L)$ generated by all $x \otimes y-y \otimes x-[x, y]$ with $x, y \in L$. Define $\mathfrak{U}(L)=\mathfrak{T}(L) / J$, let $\pi: \mathfrak{T}(L) \rightarrow \mathfrak{U}(L)$ be the canonical homomorphism and $i=\pi_{\mid L}$. Then the following theorem holds:

Theorem 4.1.2. $(\mathfrak{U}(L), i)$ is the universal enveloping algebra for $L$.

Proof. Let $\mathfrak{A}$ be an associative algebra with 1 and $j: L \rightarrow \mathfrak{A}_{L}$ a homomorphism of Lie algebras. We want to show that there exists a unique homomorphism of algebras $j^{\prime}: \mathfrak{U}(L) \rightarrow \mathfrak{A}$ such that $j^{\prime} \circ i=j$. By the universal property of $\mathfrak{T}(L)$ there exists a homomorphism of algebras $j^{\prime \prime}$ : $\mathfrak{T}(L) \rightarrow \mathfrak{A}$. Let, now, $x, y$ be in $L$. Then $j^{\prime \prime}(x \otimes y-y \otimes x-[x, y])=$ $j^{\prime \prime}(x) j^{\prime \prime}(y)-j^{\prime \prime}(y) j^{\prime \prime}(x)-j^{\prime \prime}([x, y])$ because $j^{\prime \prime}$ is a homomorphism of algebras, but $j^{\prime \prime}(x)=j(x), j^{\prime \prime}(y)=j(y), j^{\prime \prime}([x, y])=j([x, y])$ since $x, y,[x, y] \in L$ and $j([x, y])=[j(x), j(y)]$ because $j$ is a homomorphism of Lie algebras. Hence $x \otimes y-y \otimes x-[x, y]$ lies in $K e r j^{\prime \prime}$ and $j^{\prime \prime}$ induces a homomorphism of algebras $j^{\prime}: \mathfrak{U}(L) \rightarrow \mathfrak{A}$ such that $j^{\prime} \circ i=j$. We have now to prove the uniqueness of such a homomorphism. The tensor algebra $\mathfrak{T}(L)$ is generated by 1 and $L$ and this implies that $\mathfrak{U}(L)$ is generated by 1 and $i(L)$. Two homomorphisms which are identical on a set of generators are necessarily the same homomorphism. Hence uniqueness holds. 
From now on, given a Lie algebra $L$ and thanks to point 1 of the Theorem (4.1.1) and thanks to the Theorem (4.1.2), we will identify the universal enveloping algebra of $L$ with $\mathfrak{U}(L)$.

Remark 35. Since the two sided ideal $J$ is generated by all $x \otimes y-y \otimes x-$ $[x, y]$ where $x, y \in L, J$ is included in $\bigoplus_{i>0} T^{i}(L)$ and $\pi$ maps $T^{0}(L)=F$ isomorphically into $\mathfrak{U}(L)$ so $\mathfrak{U}(L)$ contains at least the scalars.

Remark 36. Let $\varphi: L \rightarrow \mathfrak{g l}(V)$ be a representation of $L$ on $V$. Then, according to the defining property (3) of the universal enveloping algebra, we can extend $\varphi$ in a unique way to a homomorphism of algebras $\phi: \mathfrak{U}(L) \rightarrow$ $\operatorname{End}(V)$. On the other hand given a homomorphism of algebras $\phi: \mathfrak{U}(L) \rightarrow$ $\operatorname{End}(V)$, we trivially have a representation of $L$ on $V$ given by the map $\phi \circ i$ which is also a homomorphism of Lie algebras. Hence, studying $L$ representations is the same as studying $\mathfrak{U}(L)$-representations with, in the latter case, the advantage of working with an associative structure.

\subsection{Poicaré-Birkhoff-Witt Theorem}

We shall now prove the Poincaré-Birkhoff-Witt Theorem which gives us the structure of the universal enveloping algebra of a Lie algebra. We denote by $x_{i_{1}} x_{i_{2}} \cdots x_{i_{n}}$ the image of $x_{i_{1}} \otimes x_{i_{2}} \otimes \cdots \otimes x_{i_{n}}$ in $\mathfrak{U}(L)=\mathfrak{T}(L) / J$.

Theorem 4.2.1. Let $\left\{x_{i}, i \in I\right\}$, where $I$ is an ordered set, be a basis of a Lie algebra $L$. Then the monomials

$$
x_{i_{1}}^{m_{1}} x_{i_{2}}^{m_{2}} \cdots x_{i_{n}}^{m_{n}}, \quad i_{1}<\ldots<i_{n}, m_{i} \in \mathbb{Z}_{+}
$$

form a basis for the universal enveloping algebra $\mathfrak{U}(L)$.

Proof. We know that $\left\{x_{i_{1}} \otimes x_{i_{2}} \otimes \cdots \otimes x_{i_{n}}, i_{1}, i_{2}, \ldots, i_{n} \in I\right\}$ is a basis of $\mathfrak{T}(L)$, therefore the images of these elements in $\mathfrak{U}(L)$ generate $\mathfrak{U}(L)$, but what is new is that the images of monomials with ordered indices generate $\mathfrak{U}(L)$. Seeking Jacobson notation, we refer to the monomials in $\mathfrak{T}(L)$ with ordered indices 
as standard monomials. We want to see that $x_{i_{1}} x_{i_{2}} \ldots x_{i_{n}}$ with arbitrary $i_{1}, i_{2}, \ldots, i_{n}$ in $I$ can be rewritten as a sum of images of standard monomials. Let $x_{i_{1}} x_{i_{2}} \ldots x_{i_{n}}$ be with indices not correctly ordered. Then there is at least one index $j$ such that $i_{j}>i_{j+1}$, but $x_{i_{j}} x_{i_{j+1}}-x_{i_{j+1}} x_{i_{j}}-\left[x_{i_{j}}, x_{i_{j+1}}\right]$ lies in J so

$$
\begin{gathered}
x_{i_{1}} x_{i_{2}} \ldots x_{i_{n}}=x_{i_{1}} \cdots x_{i_{j-1}}\left(x_{i_{j}} x_{i_{j+1}}-x_{i_{j+1}} x_{i_{j}}-\left[x_{i_{j}}, x_{i_{j+1}}\right]\right) x_{i_{j+2}} \cdots x_{i_{n}}+ \\
\quad+x_{i_{1}} \cdots x_{i_{j-1}} x_{i_{j+1}} x_{i_{j}} x_{i_{j+2}} \cdots x_{i_{n}}+x_{i_{1}} \cdots x_{i_{j-1}}\left[x_{i_{j}}, x_{i_{j+1}}\right] x_{i_{j+2}} \cdots x_{i_{n}} .
\end{gathered}
$$

Note that the first summand of the right-hand side is 0 , the second summand has a lower number of couples of indices out of order and the third is of lower degree (it is the product of $n-1$ elements of $L$ instead of $n$ elements). Hence by a double induction first on the degree, then, for each fixed degree, on the pairs of indices not correctly ordered, we are able to see that the monomials $x_{i_{1}} \cdots x_{i_{n}}$ with $i_{1} \leq i_{2} \leq \ldots \leq i_{n}$ in $I$ generate $\mathfrak{U}(L)$.

Now let us prove the linear independence of the images of standard monomials. Given a tensor monomial $x_{i_{1}} \otimes \cdots \otimes x_{i_{n}}$ we say that its defect is the number of pairs of indices $j<j^{\prime}$ such that $i_{j}>i_{j^{\prime}}$. Of course the defect is 0 if and only if the monomial is standard. Let us prove that there exists a linear map

$$
f: \mathfrak{T}(L) \rightarrow \mathfrak{T}(L)
$$

such that $f$ is the identity map on standard monomials and

$$
\begin{aligned}
f\left(x_{i_{1}} \otimes \cdots \otimes x_{i_{n}}\right)= & f\left(x_{i_{1}} \otimes \cdots \otimes x_{i_{j+1}} \otimes x_{i_{j}} \otimes \cdots \otimes x_{i_{n}}\right) \\
& +f\left(x_{i_{1}} \otimes \cdots \otimes\left[x_{i_{j}}, x_{i_{j+1}}\right] \otimes \cdots \otimes x_{i_{n}}\right) .
\end{aligned}
$$

We define this mapping by induction first on $n$, the degree of tensors, and, for each fixed degree, by induction on the defect. If $n=0,1$ the mapping is well defined because all the monomials is these cases are standard. So $f$ is defined on $T^{0}(L)+T^{1}(L)$. Fix $n \geq 2$ and define $f\left(x_{i_{1}} \otimes \cdots \otimes x_{i_{n}}\right)$ using (4.3):

$$
\begin{aligned}
f\left(x_{i_{1}} \otimes \cdots \otimes x_{i_{n}}\right)= & f\left(x_{i_{1}} \otimes \cdots \otimes x_{i_{j+1}} \otimes x_{i_{j}} \otimes \cdots \otimes x_{i_{n}}\right) \\
& +f\left(x_{i_{1}} \otimes \cdots \otimes\left[x_{i_{j}}, x_{i_{j+1}}\right] \otimes \cdots \otimes x_{i_{n}}\right) .
\end{aligned}
$$

The second summand in the right-hand side has lower degree than $x_{i_{1}} \otimes$ $\cdots \otimes x_{i_{n}}$ and the first one has smaller defect. We need to prove that the 
map is well defined because of the sequence of applications of (4.3): so we have to prove that (4.3) is independent of the choice of the pair $\left(j_{k}, j_{k+1}\right)$. If the defect of the monomial is 0 then the monomial is standard and $f$ is well defined; if the defect is $1, f$ is well defined too by (4.3) because, if $j$ is such that $i_{j}>i_{j+1}, x_{i_{1}} \otimes \cdots \otimes x_{i_{j+1}} \otimes x_{i_{j}} \otimes \cdots \otimes x_{i_{n}}$ is standard and $x_{i_{1}} \otimes \cdots \otimes\left[x_{i_{j}}, x_{i_{j+1}}\right] \otimes \cdots \otimes x_{i_{n}}$ has degree $n-1$. Suppose that the defect is 2 or greater. Let $j, j^{\prime}$, with $j<j^{\prime}$, be two indices such that $i_{j}>i_{j+1}$ and $i_{j^{\prime}}>i_{j^{\prime}+1}$, then there are essentially two cases: $j^{\prime}>j+1$ and $j^{\prime}=j+1$. If we are in the first case, setting $x_{i_{j}}=a, x_{i_{j+1}}=b, x_{i_{j^{\prime}}}=c$ and $x_{i_{j^{\prime}+1}}=d$ we have

$$
\begin{array}{r}
f(\ldots \otimes a \otimes b \otimes \ldots \otimes c \otimes d \otimes \ldots)= \\
f(\ldots \otimes b \otimes a \otimes \ldots \otimes c \otimes d \otimes \ldots)+f(\ldots \otimes[a, b] \otimes \ldots \otimes c \otimes d \otimes \ldots)
\end{array}
$$

where we applied (4.3) to $a \otimes b$. Then, appling (4.3) to $c \otimes d$ we obtain

$$
\begin{array}{r}
\quad f(\ldots \otimes b \otimes a \otimes \ldots \otimes d \otimes c \otimes \ldots)+f(\ldots \otimes b \otimes a \otimes \ldots \otimes[c, d] \otimes \ldots) \\
+f(\ldots \otimes[a, b] \otimes \ldots \otimes d \otimes c \otimes \ldots)+f(\ldots \otimes[a, b] \otimes \ldots \otimes[c, d] \otimes \ldots) .
\end{array}
$$

If, instead, we apply (4.3) first to $c \otimes d$ and then to $a \otimes b$, we have:

$$
\begin{aligned}
& f(\ldots \otimes a \otimes b \otimes \ldots \otimes d \otimes c \otimes \ldots)+f(\ldots \otimes a \otimes b \otimes \ldots \otimes[c, d] \otimes \ldots) \\
= & f(\ldots \otimes b \otimes a \otimes \ldots \otimes d \otimes c \otimes \ldots)+f(\ldots \otimes[a, b] \otimes \ldots \otimes d \otimes c \otimes \ldots) \\
& +f(\ldots \otimes b \otimes a \otimes \ldots \otimes[c, d] \otimes \ldots)+f(\ldots \otimes[a, b] \otimes \ldots \otimes[c, d] \otimes \ldots)
\end{aligned}
$$

which is the same expression obtained above.

If we are in the second case and we set $x_{i_{j}}=a, x_{i_{j+1}}=b=x_{i_{j^{\prime}}}$ and $x_{i_{j^{\prime}+1}}=c$, we have

$$
\begin{array}{r}
f(\ldots \otimes a \otimes b \otimes c \otimes \ldots)=f(\ldots \otimes b \otimes a \otimes c \otimes \ldots)+f(\ldots \otimes[a, b] \otimes c \otimes \ldots) \\
=f(\ldots \otimes b \otimes c \otimes a \otimes \ldots)+f(\ldots \otimes b \otimes[a, c] \otimes \ldots)+f(\ldots \otimes[a, b] \otimes c \otimes \ldots) \\
=f(\ldots \otimes c \otimes b \otimes a \otimes \ldots)+f(\ldots \otimes[b, c] \otimes a \otimes \ldots)+ \\
+\ldots \otimes b \otimes[a, c] \otimes \ldots) \\
+f(\ldots \otimes[a, b] \otimes c \otimes \ldots)
\end{array}
$$


on the other hand, appling (4.3) first to $b \otimes c$ we have

$f(\ldots \otimes a \otimes b \otimes c \otimes \ldots)=f(\ldots \otimes a \otimes c \otimes b \otimes \ldots)+f(\ldots \otimes a \otimes[b, c] \otimes \ldots)$

$=f(\ldots \otimes c \otimes a \otimes b \otimes \ldots)+f(\ldots \otimes[a, c] \otimes b \otimes \ldots)+f(\ldots \otimes a \otimes[b, c] \otimes \ldots)$

$=f(\ldots \otimes c \otimes b \otimes a \otimes \ldots)+f(\ldots \otimes c \otimes[a, b] \otimes \ldots)+f(\ldots \otimes[a, c] \otimes b \otimes \ldots)$

$+f(\ldots \otimes a \otimes[b, c] \otimes \ldots)$.

Then appling (4.3) to the last three summand

$$
\begin{array}{r}
f(\ldots \otimes c \otimes b \otimes a \otimes \ldots)+f(\ldots \otimes[a, b] \otimes c \otimes \ldots)+ \\
+f(\ldots \otimes b \otimes[a, c] \otimes \ldots) \\
+f(\ldots \otimes[b, c] \otimes a \otimes \ldots)+f(\ldots \otimes[c,[a, b]] \otimes \ldots)+f(\ldots \otimes[[a, c], b] \otimes \ldots) \\
+f(\ldots \otimes[a,[b, c]] \otimes \ldots)
\end{array}
$$

$=f(\ldots \otimes c \otimes b \otimes a \otimes \ldots)+f(\ldots \otimes[a, b] \otimes c \otimes \ldots)+f(\ldots \otimes b \otimes[a, c] \otimes \ldots)$

$$
+f(\ldots \otimes[b, c] \otimes a \otimes \ldots)+f(\ldots \otimes([c,[a, b]]+[[a, c], b]+[a,[b, c]]) \otimes \ldots)
$$

but $[c,[a, b]]+[[a, c], b]+[a,[b, c]]=[c,[a, b]]+[b,[c, a]]+[a,[b, c]]=0$ due to Jacobi identity, therefore the expressions

$$
\begin{aligned}
f(\ldots \otimes c \otimes b \otimes a \otimes \ldots)+f(\ldots \otimes[b, c] \otimes a \otimes \ldots) & +f(\ldots \otimes b \otimes[a, c] \otimes \ldots) \\
& +f(\ldots \otimes[a, b] \otimes c \otimes \ldots)
\end{aligned}
$$

and

$$
\begin{aligned}
& f(\ldots \otimes c \otimes b \otimes a \otimes \ldots)+f(\ldots \otimes[a, b] \otimes c \otimes \ldots)+f(\ldots \otimes b \otimes[a, c] \otimes \ldots) \\
& \quad+f(\ldots \otimes[b, c] \otimes a \otimes \ldots)+f(\ldots \otimes([c,[a, b]]+[[a, c], b]+[a,[b, c]]) \otimes \ldots)
\end{aligned}
$$

are the same and $f$ is well defined.

Due to (4.3), $f(J)=0$ so $f$ induces a linear map

$$
\phi: \mathfrak{U}(L)=\mathfrak{T}(L) / J \rightarrow \mathfrak{T}(L)
$$

such that, given $i_{1} \leq i_{2} \leq \ldots \leq i_{n}$ in $I$,

$$
\begin{array}{r}
\phi\left(x_{i_{1}} x_{i_{2}} \cdots x_{i_{n}}\right)=\phi\left(\left[x_{i_{1}} \otimes x_{i_{2}} \otimes \cdots \otimes x_{i_{n}}\right]\right) \\
=f\left(x_{i_{1}} \otimes x_{i_{2}} \otimes \cdots \otimes x_{i_{n}}\right)=x_{i_{1}} \otimes x_{i_{2}} \otimes \cdots \otimes x_{i_{n}} .
\end{array}
$$


The mapping is well defined because if we take another representative of the class $\left[x_{i_{1}} \otimes x_{i_{2}} \otimes \cdots \otimes x_{i_{n}}\right]$ they differ for an element in $J$ so they are mapped to the same element. Moreover $x_{i_{1}} \otimes x_{i_{2}} \otimes \cdots \otimes x_{i_{n}}$ with $i_{1} \leq i_{2} \leq \ldots \leq i_{n}$ in $I$ are linearly independent hence taken a trivial linear combination of $x_{i_{1}} x_{i_{2}} \cdots x_{i_{n}}$ with $i_{1} \leq i_{2} \leq \ldots \leq i_{n}$ in $I$,

$$
\sum_{i_{1} \leq i_{2} \leq \ldots \leq i_{n_{l}} \in I} a_{i_{1}, i_{2}, \ldots, i_{n_{l}}} x_{i_{1}} x_{i_{2}} \cdots x_{i_{n_{l}}}=0
$$

its image under $\phi$ is

$$
\sum_{i_{1} \leq i_{2} \leq \ldots \leq i_{n_{l}} \in I} a_{i_{1}, i_{2}, \ldots, i_{n_{l}}} x_{i_{1}} \otimes x_{i_{2}} \otimes \cdots \otimes x_{i_{n_{l}}}=0
$$

and $a_{i_{1}, i_{2}, \ldots, i_{n_{l}}}=0$ due to the linear independence of $x_{i_{1}} \otimes x_{i_{2}} \otimes \cdots \otimes x_{i_{n_{l}}}$, thus $x_{i_{1}} x_{i_{2}} \cdots x_{i_{n}}$ are linearly independent.

As just seen, in the proof of Poincaré-Birkhoff-Witt Theorem we have strongly used the Lie structure of $L$.

Corollary 4.2.2. The natural mapping $i: L \rightarrow \mathfrak{T}(L) \rightarrow \mathfrak{U}(L)$ such that $i\left(x_{j}\right)=x_{j}$ is injective.

Remark 37. Due to the Corollary (4.2.2), we can consider $L$ as a Lie subalgebra of $\mathfrak{U}(L)_{L}$. 


\section{Chapter 5}

\section{Lie algebras representations}

Throughout this chapter $L$ is a semisimple Lie algebra over an algebraically closed field $\mathbb{F}$ of characteristic $0, H$ is a fixed Cartan subalgebra of $L$ (i.e. a maximal toral subalgebra of $L$ ), $\Phi$ is the corresponding root system and $\Delta$ is a base of $\Phi$.

Lemma 5.0.3. 1. $N(\Delta)=\bigoplus_{\alpha \in \Phi^{+}} L_{\alpha}$ is a nilpotent subalgebra of $L$.

2. $B(\Delta)=H \oplus \bigoplus_{\alpha \in \Phi^{+}} L_{\alpha}$ is a solvable subalgebra of $L$.

Proof. Let $x_{\alpha} \in L_{\alpha}, x_{\beta} \in L_{\beta}, h \in H$. Then, by Jacobi identity, $\left[h,\left[x_{\alpha}, x_{\beta}\right]\right]=$ $-\left[x_{\alpha},\left[x_{\beta}, h\right]\right]-\left[x_{\beta},\left[h, x_{\alpha}\right]\right]=-\left[x_{\alpha},-\beta(h) x_{\beta}\right]-\left[x_{\beta}, \alpha(h) x_{\alpha}\right]=\beta(h)\left[x_{\alpha}, x_{\beta}\right]+$ $\alpha(h)\left[x_{\alpha}, x_{\beta}\right]=(\alpha+\beta)(h)\left[x_{\alpha}, x_{\beta}\right]$, i.e. $\left[x_{\alpha}, x_{\beta}\right] \in L_{\alpha+\beta}$. Therefore $N(\Delta)$ and $B(\Delta)$ are Lie subalgebras of $L$ because, if $\alpha, \beta \in \Phi^{+}$and $\alpha+\beta \in \Phi$, $\alpha+\beta \in \Phi^{+}$.

1) $[N(\Delta), N(\Delta)]=\sum_{\alpha, \beta \in \Phi^{+}}\left[L_{\alpha}, L_{\beta}\right]=\sum_{\alpha, \beta \in \Phi^{+}} L_{\alpha+\beta}$, but $\Phi^{+}$is finite so there exists an $i$ such that $\left[N(\Delta)^{i}, N(\Delta)\right]=0$ that is $N(\Delta)$ is nilpotent.

$2)[B(\Delta), B(\Delta)]=[H, H]+[H, N(\Delta)]+[N(\Delta), H)]+[N(\Delta), N(\Delta)]$, the first summand is 0 and the last three summands are in $N(\Delta)$ which is nilpotent. So $[B(\Delta), B(\Delta)]$ is nilpotent and $B(\Delta)$ is solvable.

Proposition 5.0.4. Let $L$ be a semisimple Lie algebra and let $\phi: L \rightarrow \mathfrak{g l}(V)$ be a representation of $L$ on a vector space $V$. If $x \in L$ is a semisimple element 
then $\phi(x)$ is a semisimple element of $\mathfrak{g l}(V)$. If $x \in L$ is a nilpotent element then $\phi(x)$ is a nilpotent element of $\mathfrak{g l}(V)$.

Proof. Since $x$ is a semisimple element of $L, a d_{x}$ is semisimple, therefore there exists a basis of eigenvectors $\left\{x_{1}, \ldots, x_{n}\right\}$ of $L$ with respect to $a d_{x}$. $a d_{\phi(x)}\left(\phi\left(x_{i}\right)\right)=\left[\phi(x), \phi\left(x_{i}\right)\right]=\phi\left(\left[x, x_{i}\right]\right)=\phi\left(a d_{x}\left(x_{i}\right)\right)=\phi\left(\lambda_{i} x_{i}\right)=\lambda_{i} \phi\left(x_{i}\right)$ because $\phi$ is a homomorphism of Lie algebras. Therefore $\phi\left(x_{1}\right), \ldots, \phi\left(x_{n}\right)$ are eigenvectors and they generate $\phi(L)$, i.e. $a d_{\phi(x)}$ is semisimple because we can choose those $\phi\left(x_{i}\right)$ which are linearly independent and we obtain a basis of eigenvectors. Let us now consider a nilpotent element $x \in L$. Since $a d_{\phi(x)}(\phi(y))=\phi\left(a d_{x} y\right)$ by induction $\left(a d_{\phi(x)}\right)^{m}(\phi(y))=\phi\left(\left(a d_{x}\right)^{m} y\right)$. Hence $a d_{\phi(x)}$ is nilpotent because by $x$-nilpotence there exists some $i \in \mathbb{Z}_{+}$such that $\left(a d_{x}\right)^{i}=0$ then $\left(a d_{\phi(x)}\right)^{i}(\phi(y))=\phi\left(\left(a d_{x}\right)^{i} y\right)=0$ for all $y \in L$.

Let $V$ be a finite dimensional $L$-module and let $\phi: L \rightarrow \mathfrak{g l}(V)$ be the representation associated to the $L$-module $V$. Thanks to Proposition (5.0.4), the set $\phi(H)$ consists of semisimple elements, moreover $\phi(H)$ is abelian, therefore the elements of $\phi(H)$ are commuting semisimple endomorphisms hence they are simultaneously diagonalizable, i.e. $H$ acts diagonally on $V$. Then $V=\sum V_{\lambda}$ where $\lambda \in H^{*}$ and $V_{\lambda}=\{v \in V \mid h . v=\lambda(h) v$ for all $h \in H\}$. If $V_{\lambda} \neq 0$ we say that $V_{\lambda}$ is a weight space and $\lambda$ is a weight of $V$.

Remark 38. The sum of weight spaces is direct.

Proof. Let $V^{\prime}=\sum_{i} V_{\mu_{i}}$ and let us suppose that the sum is not direct. Therefore there exist $v_{1}, \ldots, v_{s} \in V^{\prime}$, with $v_{i} \in V_{\mu_{i}}$ and $\mu_{i} \neq \mu_{j}$ if $i \neq j$, such that $v_{1}+\ldots+v_{s}=0$. Let us suppose moreover that $s$ is the minimum number of elements in $V^{\prime}$ such that $v_{1}+\ldots+v_{s}=0$. Since $\mu_{1} \neq \mu_{2}$, there exists some $h \in H$ such that $\mu_{1}(h) \neq \mu_{2}(h) .0=h .\left(v_{1}, \ldots, v_{s}\right)-\mu_{1}(h)\left(v_{1}, \ldots, v_{s}\right)=$ $\left(\mu_{1}(h)-\mu_{1}(h)\right) v_{1}+\left(\mu_{2}(h)-\mu_{1}(h)\right) v_{2}+\ldots+\left(\mu_{s}(h)-\mu_{1}(h)\right) v_{s}=\left(\mu_{2}(h)-\right.$ $\left.\mu_{1}(h)\right) v_{2}+\ldots+\left(\mu_{s}(h)-\mu_{1}(h)\right) v_{s}$ and $\left(\mu_{2}(h)-\mu_{1}(h)\right) v_{2} \neq 0$ by the choice of $h$. Since $\left(\mu_{i}(h)-\mu_{1}(h)\right) v_{i}$ is 0 or is an eigenvector $\left(\mu_{2}(h)-\mu_{1}(h)\right) v_{2}+\ldots+$ $\left(\mu_{s}(h)-\mu_{1}(h)\right) v_{s}$ is a sum of $m$ eigenvectors, with $1 \leq m \leq s-1$, but this is against the hypothesis of minimality of $s$. Hence the sum is direct. 
Remark 39. The sum of all the weight spaces of $V$ is an $L$-submodule.

Proof. Let $V^{\prime}$ be the sum of all the weight spaces of $V$. If $x_{\alpha} \in L_{\alpha}, h \in H$ and $v \in V_{\lambda}$, then $h \cdot x_{\alpha} \cdot v=\left[h, x_{\alpha}\right] \cdot v+x_{\alpha} \cdot h \cdot v=\alpha(h) x_{\alpha} \cdot v+\lambda(h) x_{\alpha} \cdot v=$ $(\alpha(h)+\lambda(h)) x_{\alpha} \cdot v=(\alpha+\lambda)(h) x_{\alpha} \cdot v$, i.e. $x_{\alpha} \cdot v \in V_{\lambda+\alpha} \subseteq V^{\prime}$.

If $\operatorname{dim} V=\infty$ Weyl's Theorem does not hold, nevertheless we can still define weight spaces in the same way, the sum of weight spaces, $V^{\prime}$, is always a direct sum and it is also a submodule of $\mathrm{V}$. Hence we proved the following result:

Lemma 5.0.5. Let $V$ be an L-module. Then

1. Given $\alpha \in \Phi, \lambda \in H^{*}, L_{\alpha}$ maps $V_{\lambda}$ into $V_{\lambda+\alpha}$.

2. The sum $V^{\prime}=\sum_{\lambda \in H^{*}} V_{\lambda}$ is direct and $V^{\prime}$ is an L-submodule of $V$.

3. If $\operatorname{dim} V<\infty$, then $V=V^{\prime}$.

\subsection{Standard cyclic modules}

Definition 5.1. Let $V$ be an $L$-module, then $v^{+} \in V_{\lambda}$ is called a maximal vector of weight $\lambda$ if it is a nonzero vector killed by all $L_{\alpha}$ for $\alpha \in \Phi^{+}$, i.e. $x_{\alpha} \cdot v^{+}=0$ for every $\alpha \in \Phi^{+}$.

Remark 40. It is clear that the notion of maximal vector depends strongly on the choice of the base $\Delta$.

Remark 41. If $\operatorname{dim} V<\infty, V$ contains at least one maximal vector.

Proof. Let $\phi: L \rightarrow \mathfrak{g l}(V)$ be the representation of $L$ on $V$ associated to the $L$-module $V$. By Lemma (5.0.3), $B(\Delta)=H \oplus \bigoplus_{\alpha \in \Phi^{+}} L_{\alpha}$ is a solvable subalgebra of $L$, thus $\phi(B(\Delta))$, which is isomorphic to $B(\Delta) / \operatorname{Ker}(\phi)$, is a solvable subalgebra of $\mathfrak{g l}(V)$. Moreover $\operatorname{dim} V<\infty$, therefore, by Lie's Theorem, $V$ contains a common eigenvector of all endomorphisms in $\phi(B(\Delta))$, i.e. there exist $\lambda \in(B(\Delta))^{*}$ and a nonzero vector $v$ such that $x \cdot v=\lambda(x) v$ 
for all $x \in B(\Delta)$. On the other hand $N(\Delta)=\bigoplus_{\alpha \in \Phi^{+}} L_{\alpha}$ is nilpotent thus every endomorphism of $\phi(N(\Delta))$ is nilpotent and all its eigenvectors are 0 . Hence $\lambda(x)=0$ for all $x \in N(\Delta)$ and $v$ is a maximal vector.

Remark 42. If $\operatorname{dim} V=\infty$ there is no need for a maximal vector to exist.

Example 5.1. If $L$ is simple and $\gamma$ is the highest root relative to $\Delta$, then any vector in $L_{\gamma}$ is a maximal vector for the adjoint representation.

Proof. Let $x$ be a vector in $L_{\gamma}$, then, for all $\alpha \in \Phi^{+}, x_{\alpha} \cdot x=\left[x_{\alpha}, x\right] \in L_{\alpha+\gamma}=$ 0 since $\gamma$ is the highest root.

Definition 5.2. An $L$-module $V=\mathfrak{U}(L) \cdot v^{+}$, where $v^{+}$is a maximal vector, is called a standard cyclic module and the weight of $v^{+}$is called the highest weight of $V$.

Theorem 5.1.1. Let $V$ be a standard cyclic L-module with maximal vector $v^{+} \in V_{\lambda}$ and let $\Phi^{+}=\left\{\beta_{1}, \ldots, \beta_{m}\right\}$. Then:

1. $V=\left\langle y_{\beta_{1}}^{r_{1}} y_{\beta_{2}}^{r_{2}} \cdots y_{\beta_{m}}^{r_{m}} \cdot v^{+} \mid r_{i} \in \mathbb{Z}_{+}\right\rangle, y_{\beta_{i}} \in E_{-\beta_{i}}$.

2. $V=\bigoplus_{\mu \in H^{*}} V_{\mu}$.

3. The weights of $V$ are of the form $\mu=\lambda-\sum_{\alpha_{i} \in \Delta} k_{i} \alpha_{i}$ with $k_{i} \in \mathbb{Z}_{+}$, i.e. all weights satisfy $\mu \prec \lambda$, in particular $\operatorname{dim} V_{\mu}<\infty, \operatorname{dim} V_{\lambda}=1$.

4. Every submodule $W$ of $V$ is direct sum of weight spaces.

5. $V$ is an indecomposable L-module with a unique maximal submodule and a unique irreducible quotient.

6. Every nonzero homomorphic image of $V$ is also standard cyclic of weight $\lambda$.

Proof. 1) Given a basis $\mathcal{B}=\left\{y_{\beta_{1}}, \ldots, y_{\beta_{m}}, h_{1}, \ldots, h_{l}, x_{\beta_{1}}, \ldots, x_{\beta_{m}}\right\}$ of $L$, where $x_{\beta_{i}} \in L_{\beta_{i}}, y_{\beta_{i}} \in L_{-\beta_{i}}$ and $\left\{h_{1}, \ldots, h_{l}\right\}$ is a basis of $H$, by PoincaréBirkhoff-Witt Theorem $\left\{y_{\beta_{1}}^{r_{1}} \cdots y_{\beta_{m}}^{r_{m}} h_{1}^{t_{1}} \cdots h_{l}^{t_{l}} x_{\beta_{1}}^{s_{1}} \cdots x_{\beta_{m}}^{s_{m}} \mid t_{j}, s_{i}, r_{i} \in \mathbb{Z}_{+}\right\}$is a 
basis of $\mathfrak{U}(L)$, so $\mathfrak{U}(L) \cdot v^{+}=\left\langle y_{\beta_{1}}^{r_{1}} \cdots y_{\beta_{m}}^{r_{m}} h_{1}^{t_{1}} \cdots h_{l}^{t_{l}} x_{\beta_{1}}^{s_{1}} \cdots x_{\beta_{m}}^{s_{m}} \cdot v^{+} \mid t_{i}, s_{i}, r_{i} \in \mathbb{Z}_{+}\right\rangle$. $v^{+}$is a maximal vector then $h_{i} \cdot v^{+}=c_{i} v^{+}$with $c_{i}$ in $\mathbb{F}$ and $x_{\beta_{i}} \cdot v^{+}=0$ for all $\beta_{i}$ thus $\mathfrak{U}(L) \cdot v^{+}=\left\langle y_{\beta_{1}}^{r_{1}} \cdots y_{\beta_{m}}^{r_{m}} \cdot v^{+}\right\rangle$

2) Since $h . y_{\beta_{1}}^{p_{1}} \cdots y_{\beta_{m}}^{p_{m}} \cdot v^{+}=\left(\lambda-p_{1} \beta_{1}-\ldots-p_{m} \beta_{m}\right) y_{\beta_{1}}^{p_{1}} \cdots y_{\beta_{m}}^{p_{m}} \cdot v^{+}$because $h \cdot y_{\beta} \cdot v^{+}=\left[h, y_{\beta}\right] \cdot v^{+}+y_{\beta} \cdot h \cdot v^{+}=-\beta(h) y_{\beta} \cdot v^{+}+\lambda(h) y_{\beta} \cdot v^{+}=(\lambda-\beta)(h) y_{\beta} \cdot v^{+}$ for $h \in H, V=\sum_{\mu \in H^{+}} V_{\mu}$, that is $V=\bigoplus_{\mu \in H^{+}} V_{\mu}$ because the sum of weight spaces is direct.

3) We have just proven that weights are of the form $\mu=\lambda-\sum_{i=1}^{m} p_{i} \beta_{i}$ where $\beta_{i} \in \Phi^{+}$. Any root of $\Phi^{+}$is of the form $\beta_{i}=\sum_{\alpha_{j} \in \Delta} a_{i, j} \alpha_{j}$ with $a_{i, j} \in \mathbb{Z}_{+}$, hence $\mu=\lambda-\sum_{\alpha_{j} \in \Delta}\left(\sum_{i} a_{i, j} p_{i}\right) \alpha_{j}$ and $a_{i, j} p_{i} \in \mathbb{Z}_{+}$. Given $y_{\beta_{1}}^{p_{1}} \cdots y_{\beta_{m}}^{p_{m}} \cdot v^{+} \in V_{\mu}$, $\mu=\lambda$ if and only if $a_{i, j} p_{i}=0$ for all $j$, that is $a_{i, j}=0$ or $p_{i}=0$ for all $i$. If $p_{i}=0, y_{\beta_{i}}$ doesn't appear in $y_{\beta_{1}}^{p_{1}} \cdots y_{\beta_{m}}^{p_{m}} \cdot v^{+}$; if $p_{i} \neq 0$ then $a_{i, j}=0$ for all $i$, i.e. $\sum_{\alpha_{j}} a_{i, j} \alpha_{j}$ is not a root, but the $\beta_{i}$ 's are roots therefore $p_{i}=0$ for all $i$. It follows that $\mu=\lambda$ if and only if $y_{\beta_{1}}^{p_{1}} \cdots y_{\beta_{m}}^{p_{m}} \cdot v^{+}=v^{+} \cdot V_{\lambda}=\left\langle v^{+}\right\rangle$, i.e. $\operatorname{dim} V_{\lambda}=1$. Now let fix a weight $\mu=\lambda-\sum_{\alpha_{j} \in \Delta} s_{j} \alpha_{j}$. We want to find how many generators of $\mathfrak{U}(L) \cdot v^{+}$have this weight, i.e. how many $k_{i}$ and $a_{i, j}$ in $\mathbb{Z}_{+}$there are such that $s_{j}=\sum_{i} k_{i} a_{i, j}$. Since $0 \leq k_{i}, a_{i, j} \leq s_{j}$, there is only a finite number of $k_{i}$ and $a_{i, j}$ such that $s_{j}=\sum_{i} k_{i} a_{i, j}$ hence $\operatorname{dim} V_{\mu}<\infty$.

4) As $V$ is the direct sum of weight spaces, for a given vector $w$ in $W$ there exist $v_{1}, \ldots, v_{n}$, with $v_{i}$ in $V_{\mu_{i}}$ and $\mu_{i} \neq \mu_{j}$ for $i \neq j$, such that $w=v_{1}+\ldots+v_{n}$. Let's see that $v_{i} \in W$ for all $i$. Let us suppose that there exists a vector $w \in W$ such that $w=v_{1}+\ldots+v_{n}, v_{i} \in V_{\mu_{i}}$ and $v_{j} \notin W$ for some $j$ and let $w$ such an element with the minimum number $n$ of summands. All the summands $v_{i}$ in the expression are not in $W$ otherwise permutating indices we can suppose $w=v_{1}+\ldots+v_{k}+v_{k+1}+\ldots+v_{n}$ with the first $k$ summands such that $v_{j} \in W$ and the last $n-k$ such that $v_{j} \notin W$ but in this case $w-v_{1}-\ldots-v_{k}$ is still in $W$ and it is equal to $v_{k}+\ldots+v_{n}$ so the number of summands is less than that of $w$ and this goes against the minimality of the summands of $w$. As $\mu_{1} \neq \mu_{2}$ there exists a vector $h \in H$ such that $\mu_{1}(h) \neq \mu_{2}(h)$. The element $h . w=\mu_{1}(h) v_{1}+\cdots+\mu_{n}(h) v_{n}$ lies in $W$ because $W$ is a $L$-submodule. On the other hand $\mu_{1}(h) w \in W$ so $h . w-\mu(h) w \in W$ 
and $h . w-\mu(h) w=\left(\mu_{2}(h)-\mu_{1}(h)\right) v_{2}+\ldots+\left(\mu_{n}(h)-\mu_{1}(h)\right) v_{n}$. Therefore there is an element in $W$ which is sum of $m \leq n-1$ summands not in $W$ but this goes against the minimality of $w$. Hence all the $v_{i}$ 's lie in $W$. We have just seen that $W \subseteq \bigoplus_{\mu \in H^{*}}\left(W \cap V_{\mu}\right)$. The converse is trivial so $W=\bigoplus_{\mu \in H^{*}}\left(W \cap V_{\mu}\right)$.

5) Let us suppose that $W$ is a proper $L$-submodule. Since $W$ is a proper $L$-submodule, $v^{+} \notin W$ (otherwise the action of $L$ on $v^{+}$gives all $V$, but $W \neq V)$, i.e. $W \subseteq \bigoplus_{\mu \neq \lambda} V_{\mu}$ because $\operatorname{dim} V_{\lambda}=1$. It follows that $V$ is indecomposable because the same property holds for every proper submodule. Moreover, if $W, W^{\prime}$ are proper $L$-submodules of $V$, then $W, W^{\prime} \subseteq \oplus_{\mu \neq \lambda} V_{\mu}$, hence $W+W^{\prime}$ is a proper $L$-submodule, because $W+W^{\prime} \subseteq \oplus_{\mu \neq \lambda} V_{\mu}$. It follows that $V$ has a unique maximal $L$-submodule. Indeed if there were two distinct maximal submodules their sum would properly includes both submodules against maximality. Finally if $W$ is a maximal $L$-submodule then $V / W$ is the unique irreducible quotient of $V$.

6) Let $\phi: \mathfrak{U}(L) \cdot v^{+} \rightarrow M$ be a homomorphism of $L$-modules. By definition $\phi\left(y_{\beta_{1}}^{r_{1}} \cdots y_{\beta_{m}}^{r_{m}} \cdot v^{+}\right)=y_{\beta_{1}}^{r_{1}} \cdots y_{\beta_{m}}^{r_{m}} \phi\left(v^{+}\right)$and $\phi\left(v^{+}\right)$is a maximal vector with highest weight $\lambda$ because given an element $x_{\alpha} \in L_{\alpha}$ with $\alpha \in \Phi^{+}$, $x_{\alpha} \cdot \phi\left(v^{+}\right)=\phi\left(x_{\alpha} \cdot v^{+}\right)=0$ and $h . \phi\left(v^{+}\right)=\phi\left(h \cdot v^{+}\right)=\phi\left(\lambda(h) v^{+}\right)=\lambda(h) \phi\left(v^{+}\right)$ for every $h$ in $H$.

Corollary 5.1.2. Let $V$ be as in Theorem (5.1.1). Suppose further that $V$ is an irreducible L-module. Then $v^{+}$is the unique maximal vector in $V$, up to nonzero scalar multiples.

Proof. Let $v^{+}, w^{+}$be maximal vectors with highest weights $\lambda, \sigma$. Since $V$ is irreducible, $V=\mathfrak{U}(L) \cdot v^{+}$, so $\sigma=\lambda-\sum_{\alpha_{i} \in \Delta} k_{i} \alpha_{i}$ with $k_{i} \in \mathbb{Z}_{+}$, and $V=$ $\mathfrak{U}(L) . w^{+}$, so $\lambda=\sigma-\sum_{\alpha_{j} \in \Delta} h_{j} \alpha_{j}$ with $h_{j} \in \mathbb{Z}_{+}$. It follows that $k_{i}=h_{j}=0$, that is $\lambda=\sigma$, and $w^{+}=c v^{+}$with $c \in \mathbb{F}$ since $\operatorname{dim} V_{\lambda}=1$. 


\subsection{Existence and uniqueness of the irreducible standard cyclic module}

Our next aim is to show that there exists an irreducible standard cyclic $L$-module with highest $\lambda \in H^{*}$ which is unique up to isomorphism.

Theorem 5.2.1. Let $V, W$ be standard cyclic L-modules of highest weight $\lambda$. If $V$ and $W$ are irreducible, then they are isomorphic.

Proof. Let us consider the $L$-module $X=V \oplus W$ and the vector $x^{+}=$ $\left(v^{+}, w^{+}\right)$where $v^{+}$and $w^{+}$are maximal vectors of highest weight $\lambda$ in $V, W$ respectively. $x^{+}$is a maximal vector in $X$ because $x_{\alpha} \cdot x^{+}=\left(x_{\alpha} \cdot v^{+}, x_{\alpha} \cdot w^{+}\right)=$ 0 for all $x_{\alpha} \in L_{\alpha}$ where $\alpha \in \Phi^{+}$and $x^{+}$has highest weight $\lambda$ because $h . x^{+}=\left(h . v^{+}, h . w^{+}\right)=\left(\lambda(h) v^{+}, \lambda(h) w^{+}\right)=\lambda(h)\left(v^{+}, w^{+}\right)$for all $h \in H$. Let $Y$ be the $L$-submodule of $X$ generated by $x^{+}$. Then $Y$ is also a $\mathfrak{U}(L)$-module and it is generated by $x^{+}$so it is standard cyclic. Let us now consider the natural projections $p: Y \rightarrow V$ and $p^{\prime}: Y \rightarrow W$ which are the restrictions to $\mathrm{Y}$ of the projections onto the first and second factors. The maps $p, p^{\prime}$ are homomorphisms of $L$-modules and they are surjective because $p\left(x^{+}\right)=v^{+}$ and $p^{\prime}\left(x^{+}\right)=w^{+}$. Ker $p$ and $\operatorname{Ker} p^{\prime}$ are $L$-submodules of $Y$ and $Y / \operatorname{Ker} p \cong$ $V$ and $Y / \operatorname{Ker} p^{\prime} \cong W$ hence $Y / \operatorname{Ker} p$ and $Y / \operatorname{Ker} p^{\prime}$ are irreducible and by the uniqueness of the irreducible quotient of a standard cyclic module $Y / \operatorname{Ker} p=Y / \operatorname{Ker} p^{\prime}$ that is $V \cong W$.

Thanks to the Theorem (5.2.1), we only need to prove the existence of the irreducible standard cyclic module of weight $\lambda \in H^{*}$.

Remark 43. A standard cyclic module contains a one dimensional $B(\Delta)$ submodule spanned by its maximal vector.

Let us fix $\lambda \in H^{*}$ and let $D_{\lambda}$ be a one-dimensional vector space spanned by a vector $v^{+}$. We define the following action of $B(\Delta)$ on $D_{\lambda}$ :

$$
\begin{gathered}
h . v^{+}=\lambda(h) v^{+} \quad \forall h \in H \\
x_{\alpha} \cdot v^{+}=0 \quad \forall \alpha \in \Phi^{+} .
\end{gathered}
$$


$D_{\lambda}$ is a $B(\Delta)$-module because $x_{\alpha} \cdot x_{\beta} \cdot v^{+}-x_{\beta} \cdot x_{\alpha} \cdot v^{+}=0=c x_{\alpha+\beta} \cdot v^{+}=$ $\left[x_{\alpha}, x_{\beta}\right] \cdot v^{+}$and $h \cdot x_{\alpha} \cdot v^{+}-x_{\alpha} \cdot h \cdot v^{+}=-\lambda(h) x_{\alpha} \cdot v^{+}=0=\alpha(h) x_{\alpha} \cdot v^{+}=$ $\left[h, x_{\alpha}\right] . v^{+}$. On the other hand $D(\lambda)$ is also a $\mathfrak{U}(B(\Delta))$-module and it makes sense to consider the tensor product $Z(\lambda)=\mathfrak{U}(L) \bigotimes_{\mathfrak{U}(B(\Delta))} D_{\lambda}$ which is a $\mathfrak{U}(L)$-module under the left action of $\mathfrak{U}(L)$. Moreover $1 \otimes v^{+}$generates $Z(\lambda)$ as a $\mathfrak{U}(L)$-module, it is nonzero because $\mathfrak{U}(L)$ is a free $\mathfrak{U}(B(\Delta))$-module, it is annihilated by all the $x_{\alpha} \in L_{\alpha}$ with $\alpha \in \Phi^{+}$and the action of $h \in H$ gives $\lambda(h) 1 \otimes v^{+}$. Hence $1 \otimes v^{+}$is a maximal vector with highest weight $\lambda$, that is $Z(\lambda)$ is a standard cyclic module.

Theorem 5.2.2. Let $\lambda \in H^{*}$. Then there exists an irreducible standard cyclic module $V(\lambda)$ of weight $\lambda$.

Proof. Given $\lambda \in H^{*}$ we can construct a standard cyclic module $Z(\lambda)$ of highest weight $\lambda$ as above. Since any standard cyclic module has a unique irreducible quotient $V / W$, because of Theorem (5.1.1), 5 , and $V / W$ is standard cyclic of highest weight $\lambda$, because of Theorem (5.1.1), 6, indeed $V / W$ is a nonzero homomorphic image of $V, V / W$ is the irreducible standard cyclic module we are looking for. We will denote by $V(\lambda)$ the unique irreducible quotient $V / W$ of $Z(\lambda)$.

Let us see another result which was unthinkable before having treated the previous arguments. If $V$ is a finite dimensional irreducible $L$-module then, by Remark (41), there exists at least one maximal vector $v^{+}$, of weight $\lambda$ in $H^{*}$, which necessarily generates $V$ as an $L$-module because of the irreducibility of $V . V$ is also a $\mathfrak{U}(L)$-module and $v^{+}$generates $V$ as a $\mathfrak{U}(L)$-module. Hence $V=\mathfrak{U}(L) \cdot v^{+}$, i.e. $V$ is a standard cyclic module. Moreover $V$ is an irreducible standard cyclic module therefore, by Theorem (5.2.1) $V$ is isomorphic to $V(\lambda)$. Summarizing this argument we have:

Theorem 5.2.3. Let $V$ be a finite dimensional irreducible L-module. Then $V$ is isomorphic to $V(\lambda)$ for some $\lambda$ in $H^{*}$.

Notice that, thanks to Weyl's Theorem and Theorem (5.2.3), any finite 
dimensional $L$-module $V$ is a direct sum of irreducible standard cyclic modules $V\left(\lambda_{i}\right)$ for some $\lambda_{i} \in H^{*}$.

Example 5.2. Let $L$ be a simple Lie algebra of finite dimension. Then the adjoint representation of $L$ is irreducible, moreover $L \cong L(\alpha)$ where $\alpha \in \Phi$ is the highest root, i.e. $\alpha \in \Phi$ and $\alpha+\alpha_{i} \notin \Phi$ for all $\alpha_{i} \in \Delta$.

Example 5.3. Since the standard representation of $\mathfrak{s l}_{n}(\mathbb{F})$ is finite dimensional and irreducible, $\mathbb{F}^{n} \cong V(\lambda)$ for some weight $\lambda$. Let us recall that:

$$
\mathfrak{s l}_{n}(\mathbb{F})=H \oplus \bigoplus_{\alpha \in \Phi} \mathfrak{s l}_{n}(\mathbb{F})_{\alpha}
$$

with

- $H=\left\langle e_{i i}-e_{i+1 i+1}: 1 \leq i \leq n-1\right\rangle$,

- $\Phi=\left\{ \pm\left(\alpha_{i}+\ldots+\alpha_{j-1}\right): 1 \leq i<j \leq n\right\}=\left\{\alpha_{i j}: 1 \leq i, j \leq n, i \neq j\right\}$,

- $\mathfrak{s l}_{n}(\mathbb{F})_{\alpha_{i j}}=\left\langle e_{i j}\right\rangle$.

Moreover choosing $\Delta=\left\{\alpha_{i}=\alpha_{i i+1}\right\}$ as a base of $\Phi$ we have that:

- $\Phi^{+}=\left\{\alpha_{i}+\ldots+\alpha_{j-1}: 1 \leq i<j \leq n\right\}=\left\{\alpha_{i j}: 1 \leq i<j \leq n\right\}$,

- $\left.\Phi^{-}=\left\{-\alpha_{i}-\ldots-\alpha_{j-1}\right): 1 \leq i<j \leq n\right\}=\left\{\alpha_{i j}: 1 \leq j<i \leq n\right\}$.

Therefore $v^{+}$is a maximal vector of $\mathbb{F}^{n}$ if it is an eigenvector for every $h \in H^{*}$ and $e_{i j} v^{+}=0$ for $1 \leq i<j \leq n-1$. It follows that

$$
v^{+}=e_{1}
$$

indeed $e_{i j} e_{1}=0$ if $i<j$. In addition $\left(e_{i i}-e_{i+1 i+1}\right) e_{1}=\delta_{i 1}$ hence the highest weight $\lambda$ is such that $\lambda\left(e_{i i}-e_{i+1 i+1}\right)=\delta_{i 1}$.

\subsection{Necessary and sufficient condition for fi- nite dimension}

In Section (5.2) we identified every finite dimensional irreducible $L$-module $V$ with an irreducible standard cyclic module $V(\lambda)$ for a proper $\lambda$ in $H^{*}$. Our next aim is to decide which among the $V(\lambda)$ 's are finite dimensional. 


\subsubsection{Necessary condition for finite dimension}

Let us now suppose that $V(\lambda)$ is finite dimensional. Choosen a simple root $\alpha_{i}$ in $\Phi$, i.e. a root lying in the base $\Delta$ of $\Phi$, we can find in $V(\lambda)$ a copy of $\mathfrak{s l}_{2}(\mathbb{F}), \mathfrak{s l}_{2}\left(\alpha_{i}\right)=\left\langle x_{\alpha_{i}}, y_{\alpha_{i}},\left[x_{\alpha_{i}}, y_{\alpha_{i}}\right]=h_{\alpha_{i}}\right\rangle$, therefore we can consider $V(\lambda)$ as a $\mathfrak{s l}_{2}\left(\alpha_{i}\right)$-module. A maximal vector for the action of $L$ is also a maximal vector for the action of $\mathfrak{s l}_{2}\left(\alpha_{i}\right)$ and its weight is completely determined by the evaluation of the weight on $h_{\alpha_{i}}$, which is the base of a maximal toral subalgebra of $\mathfrak{s l}_{2}\left(\alpha_{i}\right)$. In particular $\lambda\left(h_{\alpha_{i}}\right)$ is a nonnegative integer due to the structure of the finite dimensional $\mathfrak{s l}_{2}(\mathbb{F})$-modules.

Theorem 5.3.1. Let $V$ be a finite dimensional irreducible L-module of highest weight $\lambda$. Then $\lambda\left(h_{\alpha_{i}}\right)$ is a nonnegative integer.

More precisely, thanks to the structrure of the finite dimensional $\mathfrak{s l}_{2}(\mathbb{F})$ modules, it is true that given any finite dimensional $L$-module $V$, and one of its weight $\mu$ (not necessarily a highest weight), $\mu\left(h_{\alpha_{i}}\right)=\left\langle\mu, \alpha_{i}\right\rangle \in \mathbb{Z}$ when $\alpha_{i} \in \Delta$. Therefore, recalling the definitions (1.24) and (1.25), we can say that the weights occurring in a finite dimensional representation are also weights in an abstract sense and that the highest weight $\lambda$ of $V(\lambda)$ is dominant.

Theorem 5.3.2. If $\operatorname{dim} V(\lambda)<\infty$, then $\lambda$ is a dominant weight.

We have, thus, seen that a necessary condition for $V(\lambda)$ to be finite dimensional is that $\lambda$ is dominant. Given $V(\lambda)$ with $\lambda$ dominant, what can we say about its dimension?

\subsubsection{Sufficient condition for finite dimension}

Lemma 5.3.3. Let $\alpha$ be a root in $\Phi^{+}$, let $\left\langle x_{\alpha}, y_{\alpha},\left[x_{\alpha}, y_{\alpha}\right]=h_{\alpha}\right\rangle=\mathfrak{s l}_{2}(\alpha) \cong$ $\mathfrak{s l}_{2}(\mathbb{F})$ be a copy of $\mathfrak{s l}_{2}(\mathbb{F})$ into $L$, let $\phi: L \rightarrow \mathfrak{g l}(V)$ be a representation of $L$ on $V$ such that $\phi\left(x_{\alpha}\right), \phi\left(y_{\alpha}\right)$ are locally nilpotent and let $s_{\alpha}=$ $\exp \left(\phi\left(x_{\alpha}\right)\right) \exp \left(\phi\left(-y_{\alpha}\right)\right) \exp \left(\phi\left(x_{\alpha}\right)\right)$. Then, if $v$ is in $V_{\mu}, s_{\alpha}^{-1} v$ is in $V_{\sigma_{\alpha} \mu}$. In particular $\operatorname{dim} V_{\mu}=\operatorname{dim} V_{\sigma_{\alpha} \mu}$. 
Proof. By Proposition (1.3.3), for every $k$ in $H, s_{\alpha} \phi(k) s_{\alpha}^{-1}=\phi(k-\alpha(k) h)$ then, given $v$ in $V_{\mu}, \phi(k) s_{\alpha}^{-1} v=s_{\alpha}^{-1} \phi(k-\alpha(k) h) v=s_{\alpha}^{-1}(\mu(k) v-\alpha(k) \mu(h) v)=$ $s_{\alpha}^{-1}((\mu-\mu(h) \alpha)(k) v)=(\mu-\mu(h) \alpha)(k) s_{\alpha}^{-1} v=\sigma_{\alpha} \mu(k) s_{\alpha}^{-1} v$ because $\mu(h)=$ $\langle\mu, \alpha\rangle$. Therefore $s_{\alpha}^{-1} V_{\mu} \subseteq V_{\sigma_{\alpha} \mu}$ and $\operatorname{dim} V_{\mu} \leq \operatorname{dim} V_{\sigma_{\alpha} \mu}$ because $s_{\alpha}^{-1}$ is an automorphism, thus $\operatorname{dim} V_{\mu}=\operatorname{dim} s_{\alpha}^{-1} V_{\mu}$. On the other hand $s_{\alpha}^{-1} V_{\sigma_{\alpha} \mu} \subseteq$ $V_{\sigma_{\alpha} \sigma_{\alpha} \mu}=V_{\mu}$, i.e. $\operatorname{dim} V_{\sigma_{\alpha} \mu} \leq \operatorname{dim} V_{\mu}$, hence $\operatorname{dim} V_{\mu}=\operatorname{dim} V_{\sigma_{\alpha} \mu}$.

Lemma 5.3.4. Let $\left\langle x_{i}, y_{i},\left[x_{i}, y_{i}\right]=h_{i}\right\rangle=\mathfrak{s l}_{2}\left(\alpha_{i}\right) \cong \mathfrak{s l}_{2}(\mathbb{F})$ be a copy of $\mathfrak{s l}_{2}(\mathbb{F})$ into $L$. Then the following identities hold in $\mathfrak{U}(L)$ for $k>0,1 \leq i, j \leq l$ :

1. $\left[x_{j}, y_{i}^{k+1}\right]=0$ for all $i \neq j$

2. $\left[h_{j}, y_{i}^{k+1}\right]=-(k+1) \alpha_{i}\left(h_{j}\right) y_{i}^{k+1}$

3. $\left[x_{i}, y_{i}^{k+1}\right]=-(k+1) y_{i}^{k}\left(k-h_{i}\right)$.

Proof. For any $a \in \mathfrak{U}(L)$ we have:

$\left[a, y_{i}^{k+1}\right]=a y_{i}^{k+1}-y^{k+1} a=\left(a y_{i}^{k}-y_{i}^{k} a\right) y_{i}+y_{i}^{k} a y_{i}-y^{k+1} a=\left[a, y_{i}^{k}\right] y_{i}+y_{i}^{k}\left[a, y_{i}\right]$

1) Let us prove identity (1) by induction. If $k=0,\left[x_{j}, y_{i}\right] \in L_{\alpha_{j}-\alpha_{i}}=0$ for all $i \neq j$ because $\Delta$ is a base for $\Phi$ hence $\alpha_{i}-\alpha_{j}$ is not a root. Let us suppose that $\left[x_{j}, y_{i}^{k}\right]=0$. Then, by equation (5.2), $\left[x_{j}, y_{i}^{k+1}\right]=y_{i}^{k}\left[x_{j}, y_{i}\right]=0$.

2) Let us prove identity (2) by induction. If $k=0,\left[h_{j}, y_{i}\right]=-\alpha_{i}\left(h_{j}\right) y_{i}$. Let us suppose that $\left[h_{j}, y_{i}^{k}\right]=-k \alpha_{i}\left(h_{j}\right) y_{i}^{k}$. Then, by equation $(5.2),\left[h_{j}, y_{i}^{k+1}\right]=$ $\left[h_{j}, y_{i}^{k}\right] y_{i}+y_{i}^{k}\left[h_{j}, y_{i}\right]=-k \alpha_{i}\left(h_{j}\right) y_{i}^{k} y_{i}-y_{i}^{k} \alpha_{i}\left(h_{j}\right) y_{i}=-(k+1) \alpha_{i}\left(h_{j}\right) y_{i}^{k+1}$.

3) Let us prove identity (3) by induction. If $k=0,\left[x_{i}, y_{i}\right]=h_{i}$. Let us suppose that $\left[x_{i}, y_{i}^{k}\right]=-k y_{i}^{k-1}\left(k-1-h_{i}\right)$. Then, by equation (5.2), $\left[x_{i}, y_{i}^{k+1}\right]=\left[x_{i}, y_{i}^{k}\right] y_{i}+y_{i}^{k}\left[x_{i}, y_{i}\right]=-k y_{i}^{k-1}\left(k-1-h_{i}\right) y_{i}+y_{i}^{k} h_{i}=-k y_{i}^{k-1}(k-$ 1) $y_{i}+k y_{i}^{k-1} h_{i} y_{i}+y_{i}^{k} h_{i}=-k(k-1) y_{i}^{k}+k y_{i}^{k} h_{i}+k y_{i}^{k-1}\left[h_{i}, y_{i}\right]+y_{i}^{k} h_{i}=-k(k-$ 1) $y_{i}^{k}+(k+1) y_{i}^{k} h_{i}-k y_{i}^{k-1} \alpha_{i}\left(h_{i}\right) y_{i}=-k(k+1) y_{i}^{k}+(k+1) y_{i}^{k} h_{i}=-(k+$ 1) $y_{i}^{k}\left(k-h_{i}\right)$ where we used that $\alpha_{i}\left(h_{i}\right)=2$.

Theorem 5.3.5. If $\lambda \in H^{*}$ is a dominant weight, then $V(\lambda)$ is finite dimensional. 
Proof. Let $v^{+}$be a maximal vector of weight $\lambda$ in $V(\lambda)$ and set $m_{i}=\lambda\left(h_{i}\right)$ for all $i$ such that $\alpha_{i} \in \Delta$. We want first to see that $y_{i}^{m_{i}+1}=0 . x_{j} \cdot y_{i}^{m_{i}+1} \cdot v^{+}=$ $\left[x_{j}, y_{i}^{m_{i}+1}\right] \cdot v^{+}+y_{i}^{m_{i}+1} \cdot x_{j} \cdot v^{+}=\left[x_{j}, y_{i}^{m_{i}+1}\right] \cdot v^{+}$because $v^{+}$is a maximal vector. By Lemma (5.3.4), $x_{j} \cdot y_{i}^{m_{i}+1} \cdot v^{+}=0$ if $j \neq i$ and $x_{i} \cdot y_{i}^{m_{i}+1} \cdot v^{+}=-\left(m_{i}+\right.$ 1) $y_{i}^{m_{i}}\left(m_{i}-h_{i}\right) \cdot v^{+}=0$ because $m_{i} v^{+}=\lambda\left(h_{i}\right) v^{+}=h_{i} \cdot v^{+}$. In other words $x_{j} \cdot y_{i}^{m_{i}+1} \cdot v^{+}=0$ for all $j$, i.e. either $y_{i}^{m_{i}+1} \cdot v^{+}=0$ or $y_{i}^{m_{i}+1} \cdot v^{+}$is a maximal vector. For any $h \in H, h \cdot y_{i}^{m_{i}+1} \cdot v^{+}=\left[h, y_{i}^{m_{i}+1}\right] \cdot v^{+}+y_{i}^{m_{i}+1} \cdot h \cdot v^{+}=-\left(m_{i}+\right.$ 1) $\alpha_{i}(h) y_{i}^{m_{i}+1} \cdot v^{+}+\lambda(h) y_{i}^{m_{i}+1} \cdot v^{+}=\left(\lambda-\left(m_{i}+1\right) \alpha_{i}\right)(h) y_{i}^{m_{i}+1} \cdot v^{+}$, i.e. $y_{i}^{m_{i}+1} \cdot v^{+}$ lies in $V_{\lambda-\left(m_{i}+1\right) \alpha_{i}}$. Since $m_{i}=\lambda\left(h_{i}\right)=\left\langle\lambda, \alpha_{i}\right\rangle \in \mathbb{Z}_{+}, \lambda-\left(m_{i}+1\right) \alpha_{i} \neq \lambda$, therefore $y_{i}^{m_{i}+1} \cdot v^{+}=0$ because $V(\lambda)$ is irreducible and, by corollary (5.1.2), a maximal vector of any irreducible standard cyclic module is unique up to scalar multiples.

Since $y_{i}^{m_{i}+1} \cdot v^{+}=0$, the subspace $\left\langle v^{+}, y_{i} \cdot v^{+}, \ldots, y_{i}^{m_{i}} \cdot v^{+}\right\rangle$is a $\mathfrak{s l}_{2}\left(\alpha_{i}\right)$-module because it is closed under the action of $y_{i}$, it is closed under the action of $h_{i}$ by Lemma $(5.3 .4(2))$ and $x_{i} \cdot y_{i}^{k} \cdot v^{+}=0$, if $k=0$, and, if $k>0$ $x_{i} \cdot y_{i}^{k} \cdot v^{+}=\left[x_{i}, y_{i}^{k}\right] \cdot v^{+}+y_{i}^{k} \cdot x_{i} \cdot v^{+}=\left[x_{i}, y_{i}^{k}\right] \cdot v^{+}=-k y_{i}^{k-1}\left(k-1-h_{i}\right) \cdot v^{+}=$ $-k(k-1) y_{i}^{k-1} \cdot v^{+}+\lambda\left(h_{i}\right) k y_{i}^{k-1} \cdot v^{+}=c y_{i}^{k-1} \cdot v^{+}$where $c$ is a scalar. Hence $V(\lambda)$ admits a non trivial $\mathfrak{s l}_{2}\left(\alpha_{i}\right)$-module for every $i$ such that $\alpha_{i} \in \Delta$. Let now $V^{\prime}$ be the sum of all finite dimensional $\mathfrak{s l}_{2}\left(\alpha_{i}\right)$-submodule of $V(\lambda)$. $V^{\prime} \neq 0$ because we have proved above that there is a non trivial finite dimensional $\mathfrak{s l}_{2}\left(\alpha_{i}\right)$-submodule. We want now to prove that $V^{\prime}$ is an $L$ submodule of $V(\lambda)$. Let $W$ be a $\mathfrak{s l}_{2}\left(\alpha_{i}\right)$-submodule and let us consider $W^{\prime}=\sum_{\beta \in \Phi} x_{\beta} . W$. Then $W^{\prime}$ is an $\mathfrak{s l}_{2}\left(\alpha_{i}\right)$-submodule, indeed, given a vector $w \in W, x_{i} \cdot x_{\beta} \cdot w=\left[x_{i}, x_{\beta}\right] \cdot w+x_{\beta} \cdot x_{i} \cdot w=c_{1} x_{\alpha_{i}+\beta} \cdot w+x_{\beta} \cdot w_{1}$ where $w_{1} \in W$ and $c_{1} \in \mathbb{F}, y_{i} \cdot x_{\beta} \cdot w=\left[y_{i}, x_{\beta}\right] \cdot w+x_{\beta} \cdot y_{i} \cdot w=c_{2} x_{\beta-\alpha_{i}} \cdot w+x_{\beta} \cdot w_{2}$ where $w_{2} \in W$ and $c_{2} \in \mathbb{F}$, and $h_{i} \cdot x_{\beta} \cdot w=\left[h_{i}, x_{\beta}\right] \cdot w+x_{\beta} \cdot h_{i} \cdot w=\beta\left(h_{i}\right) x_{\beta} \cdot w+x_{\beta} \cdot w_{3}$ where $w_{3} \in W$. Hence $x_{i} \cdot x_{\beta} \cdot w, y_{i} \cdot x_{\beta} \cdot w, h_{i} . x_{\beta} \cdot w \in \sum_{\beta \in \Phi} x_{\beta} . W$. In this way we have proved that a vector of any $\mathfrak{s l}_{2}\left(\alpha_{i}\right)$-submodule is sent, through the action of $L$, to a $\mathfrak{s l}_{2}\left(\alpha_{i}\right)$-submodule, hence it still lies in $V^{\prime}$, that is $V^{\prime}$ is an $L$-submodule of $V(\lambda)$. It follows by the irreducibility of $V$ that $V^{\prime}=V(\lambda)$. Note that the maps $\phi\left(x_{i}\right)$ and $\phi\left(y_{i}\right)$ are locally nilpotent therefore, by corol- 
lary (5.3.3), if $\mu$ is a weight, $\sigma_{\alpha} \mu$ is also a weight and $\operatorname{dim} V_{\mu}=\operatorname{dim} V_{\sigma_{\alpha} \mu}$. By Proposition (1.2.3), every weight is $\mathcal{W}$-conjugated to a dominant weight, but the dominant weights $\mu$ such that $\mu \prec \lambda$ are, by Lemma (1.2.4), in a finite number. Since $\mathcal{W}$ is a finite group, the number of weights of $V(\lambda)$ is finite and, as $\operatorname{dim} V_{\mu}<\infty$ for every weight $\mu$, we deduce that $\operatorname{dim} V(\lambda)$ is finite because $V(\lambda)$ is a direct sum of weight spaces. 


\section{Chapter 6}

\section{Lie superalgebras}

\subsection{Superalgebras}

Definition 6.1. Let $A$ be an algebra and $M$ an abelian group. An $M$-grading of $A$ is a decomposition of $A$ into a direct sum of subspaces $A=\oplus_{\alpha \in M} A_{\alpha}$ such that $A_{\alpha} A_{\beta} \subseteq A_{\alpha+\beta}$. An algebra equipped with an $M$-grading is called $M$-graded. If $a \in A_{\alpha}$, then we say that $a$ is homogeneous of degree $\alpha$ and we write $\operatorname{deg} a=\alpha$.

Definition 6.2. A subspace $B$ of an $M$-graded algebra $A$ is called $M$-graded if $B=\oplus_{\alpha \in M}\left(B \cap A_{\alpha}\right)$.

Remark 44. A subalgebra or an ideal of an $M$-graded algebra is an $M$-graded subalgebra or ideal.

Definition 6.3. A homomorphism $\phi: A \rightarrow A^{\prime}$ of $M$-graded algebras is a homomorphism of algebras which preserves the $M$-grading in the sense that $\phi\left(A_{\alpha}\right) \subseteq A_{\varphi(\alpha)}^{\prime}$ where $\varphi$ is an automorphism of $M$.

Let $\mathbb{Z}_{2}=\mathbb{Z} / 2 \mathbb{Z}$ be the residue class ring $\bmod 2$ with elements $\overline{0}$ and $\overline{1}$.

Definition 6.4. A superalgebra is a $\mathbb{Z}_{2}$-graded algebra $A=A_{\overline{0}} \oplus A_{\overline{1}}$. The elements of $A_{\overline{0}}$ are called even and the elements of $A_{\overline{1}}$ are called odd. Given an element $a \in A$ we denote $\operatorname{deg} a$ by $p(a)$ and we refer to $p(a)$ as the parity of $a$. 
Remark 45 . The only automorphism of groups $\varphi: \mathbb{Z}_{2} \rightarrow \mathbb{Z}_{2}$ is the identity, hence every homomorphism of superalgebras $\phi: A \rightarrow A^{\prime}$ is such that $\phi\left(A_{\alpha}\right) \subseteq$ $A_{\alpha}^{\prime}$.

Definition 6.5. A superalgebra is called

- commutative if $a b=(-1)^{p(a) p(b)} b a$

- associative if $(a b) c=a(b c)$

- anticommutative if $a b=-(-1)^{p(a) p(b)} b a$.

Example 6.1. Let $M$ be an abelian group and $V=\oplus_{\alpha \in M} V_{\alpha}$ an $M$-graded vector space. Then the associative algebra $\operatorname{End}(V)$ is naturally equipped with the induced $M$-grading $\operatorname{End}(V)=\oplus_{\alpha \in M} \operatorname{End}_{\alpha}(V)$ where

$$
\operatorname{End}_{\alpha}(V)=\left\{x \in \operatorname{End}(V) \mid x\left(V_{\beta}\right) \subseteq V_{\alpha+\beta}\right\} .
$$

In particular, for $M=\mathbb{Z}_{2}$, the associative superalgebra $\operatorname{End}(V)=\operatorname{End}_{\overline{0}}(V) \oplus$ $\operatorname{End}_{\overline{1}}(V)$.

Example 6.2. Let $\Lambda(n)$ be the Grassmann algebra in $n$ variables $\xi_{1}, \ldots, \xi_{n}$. Then, setting $p\left(\xi_{i}\right)=\overline{1}$ for all $i=1, \ldots, n$, we obtain an associative superalgebra called the Grassmann superalgebra.

\subsection{Lie superalgebras}

Definition 6.6. A Lie superalgebra is a superalgebra $\mathfrak{g}=\mathfrak{g}_{\overline{0}} \oplus \mathfrak{g}_{\overline{1}}$ with a product $[]:, \mathfrak{g} \times \mathfrak{g} \rightarrow \mathfrak{g}$ satisfying the following properties:

$$
\begin{array}{r}
{[a, b]=-(-1)^{p(a) p(b)}[b, a]} \\
{[a,[b, c]]=[[a, b], c]+(-1)^{p(a) p(b)}[b,[a, c]] .}
\end{array}
$$

The first property is the anticommutativity of the bracket and the second property is the analogue of the Jacobi identity for Lie superalgebras. 
Example 6.3. If $A$ is an associative superalgebra, then the bracket defined as follows

$$
[a, b]=a b-(-1)^{p(a) p(b)} b a
$$

gives to $A$ a structure of a Lie superalgebra.

Proof. $[a, b]=a b-(-1)^{p(a) p(b)} b a=-(-1)^{p(a) p(b)}\left(b a-(-1)^{p(a) p(b)} a b\right)=$ $-(-1)^{p(a) p(b)}[b, a]$, therefore property (6.1) holds. Moreover

$$
\begin{array}{r}
{[a,[b, c]]=\left[a, b c-(-1)^{p(b) p(c)} c b\right]=} \\
a(b c)-(-1)^{p(b) p(c)} a(c b)-(-1)^{(p(b)+p(c)) p(a)}(b c) a+ \\
(-1)^{(p(b)+p(c)) p(a)}(-1)^{p(b) p(c)}(c b) a= \\
a(b c)-(-1)^{p(b) p(c)} a(c b)-(-1)^{(p(b)+p(c)) p(a)}(b c) a+ \\
(-1)^{p(b) p(a)+p(c)(p(a)+p(b))}(c b) a
\end{array}
$$

and

$$
\begin{array}{r}
{[[a, b], c]+(-1)^{p(a) p(b)}[b,[a, c]]=} \\
{\left[a b-(-1)^{p(a) p(b)} b a, c\right]+(-1)^{p(a) p(b)}\left[b, a c-(-1)^{p(a) p(c)} c a\right]=} \\
(a b) c-(-1)^{p(a) p(b)}(b a) c- \\
(-1)^{p(c)(p(a)+p(b))} c(a b)+(-1)^{p(a) p(b)}(-1)^{p(c)(p(a)+p(b))} c(b a)+ \\
(-1)^{p(a) p(b)} b(a c)-(-1)^{p(a) p(b)}(-1)^{p(a) p(c)} b(c a)- \\
(-1)^{p(a) p(b)}(-1)^{p(b)(p(a)+p(c))}(a c) b+ \\
(-1)^{p(a) p(b)}(-1)^{p(a) p(c)}(-1)^{p(b)(p(a)+p(c))}(c a) b= \\
a(b c)-(-1)^{p(b) p(c)} a(c b)+ \\
(-1)^{p(a) p(b)+p(c)(p(a)+p(b))}(c b) a-(-1)^{p(a)(p(b)+p(c))}(b c) a
\end{array}
$$

where we use the associativity of the product in $A$ and the definition (6.3). Thus property (6.2) holds.

Remark 46. For $a, b \in \mathfrak{g}_{\overline{0}}$, properties (6.1) and (6.2) become

$$
\begin{array}{r}
{[a, b]=-[b, a]} \\
{[a,[b, c]]=[[a, b], c]+[b,[a, c]]}
\end{array}
$$


thus $\mathfrak{g}_{\overline{0}}$ is an ordinary Lie algebra.

Remark 47. For $a, b \in \mathfrak{g}_{\overline{0}}, c \in \mathfrak{g}_{\overline{1}}$, property (6.2) becomes $[[a, b], c]=[a,[b, c]]-$ $[b,[a, c]]$. Hence the operation $[$,$] restricted to \mathfrak{g}_{\overline{0}} \times \mathfrak{g}_{\overline{1}}$ gives $\mathfrak{g}_{\overline{1}}$ a $\mathfrak{g}_{\overline{0}}$-module structure.

Remark 48. The operation [, ] restricted to $\mathfrak{g}_{\overline{1}} \times \mathfrak{g}_{\overline{1}}$ is commutative because, if $a, b \in \mathfrak{g}_{\overline{1}},[a, b]=[b, a]$ hence the linear map $\varphi: S^{2} \mathfrak{g}_{\overline{1}} \rightarrow \mathfrak{g}_{\overline{0}}, \varphi(a b)=$ $[a, b]$ is well defined and $\varphi$ is a homomorphism of $\mathfrak{g}_{\overline{0}}$-modules. Indeed, given $a \in \mathfrak{g}_{\overline{0}}, b, c \in \mathfrak{g}_{\overline{1}}$ and using the definition of the action on $S^{2} \mathfrak{g}_{\overline{1}}, \varphi(a .(b c))=$ $\varphi((a . b) c+b(a . c))=\varphi([a, b] c+b[a, c])=[[a, b], c]+[b,[a, c]]=[a,[b, c]]=$ $[a, \varphi(b c)]=a \cdot(\varphi(b c))$ where we used the linearity of the map $\varphi$, the Jacobi identity (6.2), the fact that $p(a) p(b)=\overline{0}$ and the fact that $\mathfrak{g}_{\overline{1}}$ is a $\mathfrak{g}_{\overline{0}}$-module with the action given by $[$,$] .$

Moreover the following proposition holds:

Proposition 6.2.1. Let us have:

1. a Lie algebra $\mathfrak{g}_{\overline{0}}$,

2. $a \mathfrak{g}_{\overline{0}}$-module $\mathfrak{g}_{\overline{1}}$,

3. a homomorphism of $\mathfrak{g}_{\overline{0}}$-modules $\varphi: S^{2} \mathfrak{g}_{\overline{1}} \rightarrow \mathfrak{g}_{0}$ with the condition

$$
(\varphi(a b)) \cdot c+(\varphi(b c)) \cdot a+(\varphi(c a)) \cdot b=0 \text { for } a, b, c \in \mathfrak{g}_{1}
$$

where "." denotes the action of $\mathfrak{g}_{\overline{0}}$ on $\mathfrak{g}_{\overline{1}}$.

Then $\mathfrak{g}=\mathfrak{g}_{\overline{0}} \oplus \mathfrak{g}_{\overline{1}}$ is a Lie superalgebra with the product [, ] defined as follows:

- if $a, b \in \mathfrak{g}_{0},[a, b]=[a, b]$ where the latter [, ] is the one of the Lie algebra $\mathfrak{g}_{\overline{0}}$,

- if $a \in \mathfrak{g}_{\overline{0}}, b \in \mathfrak{g}_{\overline{1}},[a, b]=a . b$,

- if $a \in \mathfrak{g}_{\overline{1}}, b \in \mathfrak{g}_{\overline{0}},[a, b]=-[b, a]=-b . a$,

- if $a, b \in \mathfrak{g}_{\overline{1}},[a, b]=\varphi(a b)$. 
The definitions of solvable, nilpotent, simple and semisimple Lie superalgebras are the same as for Lie algebras, i.e:

Definition 6.7. Let $\mathfrak{g}$ be a Lie superalgebra and let $\mathfrak{g}^{(0)}=\mathfrak{g}, \mathfrak{g}^{(1)}=[\mathfrak{g}, \mathfrak{g}]$, $\mathfrak{g}^{(i)}=\left[\mathfrak{g}^{(i-1)}, \mathfrak{g}^{(i-1)}\right]$ for $i>1$. Then a Lie superalgebra $\mathfrak{g}$ is called solvable if there exists some $i \in \mathbb{Z}_{+} \cup\{0\}$ such that $\mathfrak{g}^{(i)}=0$.

Definition 6.8. Let $\mathfrak{g}$ be a Lie superalgebra and let $\mathfrak{g}^{0}=\mathfrak{g}, \mathfrak{g}^{i}=\left[\mathfrak{g}^{i-1}, \mathfrak{g}\right]$ for $i \geq 1$. Then a Lie superalgebra $\mathfrak{g}$ is called nilpotent if there exists some $i \in \mathbb{Z}_{+} \cup\{0\}$ such that $\mathfrak{g}^{i}=0$.

Definition 6.9. A Lie superalgebra $\mathfrak{g}$ is called simple if $\mathfrak{g}$ contains no nontrivial ideals.

Definition 6.10. A Lie superalgebra $\mathfrak{g}$ is called semisimple if $\mathfrak{g}$ contains no solvable ideals.

Definition 6.11. Let $\mathfrak{g}=\mathfrak{g}_{\overline{0}} \oplus \mathfrak{g}_{\overline{1}}$ and $\mathfrak{h}=\mathfrak{h}_{\overline{0}} \oplus \mathfrak{h}_{\overline{1}}$ be two Lie superalgebras. Then a linear map $\phi: \mathfrak{g} \rightarrow \mathfrak{h}$ is a homomorphism of Lie superalgebras if $\phi([x, y])=[\phi(x), \phi(y)]$.

\subsection{The superalgebra $l(V)$ and the supertrace}

Let $V=V_{\overline{0}} \oplus V_{\overline{1}}$ be a $\mathbb{Z}_{2}$-graded space. We have already seen in Example (6.1) that the algebra $\operatorname{End}(V)$ endowed with the induced $\mathbb{Z}_{2}$-grading becomes an associative superalgebra. Hence $\operatorname{End}(V)$ is a Lie superalgebra, by Example (6.3) and we denote $\operatorname{End}(V)$ with the Lie superalgebra structure by $l(V)$ or $l(m, n)$ where $m=\operatorname{dim} V_{\overline{0}}$ and $n=\operatorname{dim} V_{\overline{1}}$. Let us now consider a homogeneous basis of $V$, i.e. a basis $\left\{e_{1}, \ldots, e_{m}, e_{m+1}, \ldots, e_{m+n}\right\}$ of $V$ where $\left\{e_{1}, \ldots, e_{m}\right\}$ is a basis of $V_{\overline{0}}$ and $\left\{e_{m+1}, \ldots, e_{m+n}\right\}$ is a basis of $V_{\overline{1}}$. The matrix of an element of $l(V)$ with respect to this basis is of the form

$$
\left(\begin{array}{ll}
A & B \\
C & D
\end{array}\right)
$$


where $A$ is a $(m \times m)$-matrix, $B$ is a $(m \times n)$-matrix, $C$ is a $(n \times m)$-matrix, $D$ is a $(n \times n)$-matrix.

Note that the even part $l(V)_{\overline{0}}$ of $l(V)$ consists of matrices of the form

$$
\left(\begin{array}{ll}
A & 0 \\
0 & D
\end{array}\right)
$$

and the odd part $l(V)_{\overline{1}}$ of $l(V)$ consists of matrices of the form

$$
\left(\begin{array}{ll}
0 & B \\
C & 0
\end{array}\right)
$$

Indeed an element $a$ of $l(V)_{\overline{0}}$ is such that $a\left(V_{\overline{0}}\right) \subseteq V_{\overline{0}}$ and $a\left(V_{\overline{1}}\right) \subseteq V_{\overline{1}}$ hence its matrix with respect to an homogeneous basis has the form:

$$
\left(\begin{array}{ll}
A & 0 \\
0 & D
\end{array}\right)
$$

On the other hand an element $b$ of $l(V)_{\overline{1}}$ is such that $b\left(V_{\overline{0}}\right) \subseteq V_{\overline{1}}$ and $b\left(V_{\overline{1}}\right) \subseteq$ $V_{\overline{0}}$ hence its matrix respect to an homogeneous basis has the form:

$$
\left(\begin{array}{ll}
0 & B \\
C & 0
\end{array}\right)
$$

Definition 6.12. Let $a=\left(\begin{array}{cc}A & B \\ C & D\end{array}\right)$ be in $l(m, n)$. Then we call supertrace of $a$ the number

$$
\operatorname{str}(a)=\operatorname{tr}(A)-\operatorname{tr}(D)
$$

Remark 49. Since the trace is linear, the supertrace is linear.

Remark 50. The supertrace of the matrix of an operator $a \in l(V)$ does not depend on the choice of a homogeneous basis. Indeed, let $l \in l(V)$ and let

$$
a=\left(\begin{array}{ll}
A & B \\
C & D
\end{array}\right), a^{\prime}=\left(\begin{array}{cc}
A^{\prime} & B^{\prime} \\
C^{\prime} & D^{\prime}
\end{array}\right)
$$

be two matrices of $l$ with respect to two different homogeneous base. Then $A$ is conjugated to $A^{\prime}$ and $D$ is conjugated to $D^{\prime}$, hence $\operatorname{tr}(A)=\operatorname{tr}\left(A^{\prime}\right)$ and $\operatorname{tr}(D)=\operatorname{tr}\left(D^{\prime}\right)$. 
We now want to prove that $\operatorname{str}([a, b])=0$ for any $a, b \in l(V)$.

Definition 6.13. Let $\mathfrak{g}=\mathfrak{g}_{\overline{0}} \oplus \mathfrak{g}_{\overline{1}}$ be a $\mathbb{Z}_{2}$-graded space and let $f: \mathfrak{g} \times \mathfrak{g} \rightarrow \mathbb{F}$ be a bilinear form on $\mathfrak{g}$. Then $f$ is called consistent if $f(a, b)=0$ for any $a \in \mathfrak{g}_{\overline{0}}$ and $b \in \mathfrak{g}_{\overline{1}}, f$ is called supersymmetric if $f(a, b)=(-1)^{p(a) p(b)} f(b, a)$ and, if $\mathfrak{g}$ is a Lie superalgebra, $f$ is called invariant if $f([a, b], c)=f(a,[b, c])$.

Proposition 6.3.1. $\quad$ 1. The bilinear form $(a, b)=\operatorname{str}(a b)$ on $l(V)$ is consistent, supersymmetric and invariant.

2. $\operatorname{str}([a, b])=0$ for all $a, b \in l(V)$.

Proof. 1) (Consistency) If $a$ and $b$ are the matrices of an even and an odd element with respect to an homogeneous basis, they have the following form

$$
a=\left(\begin{array}{cc}
A & 0 \\
0 & D
\end{array}\right), b=\left(\begin{array}{cc}
0 & B \\
C & 0
\end{array}\right)
$$

hence

$$
a b=\left(\begin{array}{cc}
A & 0 \\
0 & D
\end{array}\right)\left(\begin{array}{cc}
0 & B \\
C & 0
\end{array}\right)=\left(\begin{array}{cc}
0 & A B \\
D C & 0
\end{array}\right)
$$

and $(a, b)=\operatorname{str}(a b)=0$.

(Supersimmetry) If $a \in l(V)_{\overline{0}}$ and $b \in l(V)_{\overline{1}},(a, b)=0$ via the consistency. Moreover, identifying the endomorphisms with the associated matrices respect to an homogeneous basis, we have

$$
a=\left(\begin{array}{cc}
A & 0 \\
0 & D
\end{array}\right), b=\left(\begin{array}{cc}
0 & B \\
C & 0
\end{array}\right)
$$

hence

$$
b a=\left(\begin{array}{cc}
0 & B \\
C & 0
\end{array}\right)\left(\begin{array}{cc}
A & 0 \\
0 & D
\end{array}\right)=\left(\begin{array}{cc}
0 & B D \\
C A & 0
\end{array}\right)
$$

thus $(b, a)=\operatorname{str}(b a)=0=(a, b)$. If $a, b \in l(V)_{\overline{0}}$, via the usual identifications we obtain

$$
a=\left(\begin{array}{cc}
A & 0 \\
0 & D
\end{array}\right), b=\left(\begin{array}{cc}
A^{\prime} & 0 \\
0 & D^{\prime}
\end{array}\right)
$$


therefore

$$
a b=\left(\begin{array}{cc}
A A^{\prime} & 0 \\
0 & D D^{\prime}
\end{array}\right), b a=\left(\begin{array}{cc}
A^{\prime} A & 0 \\
0 & D^{\prime} D
\end{array}\right)
$$

but $\operatorname{tr}\left(A A^{\prime}\right)=\operatorname{tr}\left(A^{\prime} A\right)$ and $\operatorname{tr}\left(D D^{\prime}\right)=\operatorname{tr}\left(D^{\prime} D\right)$ so $\operatorname{str}(a b)=\operatorname{str}(b a)$. If $a, b \in l(V)_{\overline{1}}$, via the usual identifications we obtain

$$
a=\left(\begin{array}{cc}
0 & B \\
C & 0
\end{array}\right), b=\left(\begin{array}{cc}
0 & B^{\prime} \\
C^{\prime} & 0
\end{array}\right)
$$

hence

$$
a b=\left(\begin{array}{cc}
B C^{\prime} & 0 \\
0 & C B^{\prime}
\end{array}\right), b a=\left(\begin{array}{cc}
B^{\prime} C & 0 \\
0 & C^{\prime} B
\end{array}\right)
$$

so $(a, b)=\operatorname{str}(a b)=\operatorname{tr}\left(B C^{\prime}\right)-\operatorname{tr}\left(C B^{\prime}\right)=\operatorname{tr}\left(C^{\prime} B\right)-\operatorname{tr}\left(B^{\prime} C\right)=-(\operatorname{str}(b a))=$ $(-1)^{p(b) p(a)}(b, a)$.

Let us now prove 2), then we will prove the invariance of $($,$) .$

2) $\operatorname{str}([a, b])=\operatorname{str}\left(a b-(-1)^{p(a) p(b)} b a\right)=\operatorname{str}(a b)-(-1)^{p(a) p(b)} \operatorname{str}(b a)=0$ because of the supersymmetry of the bilinear form $($,$) . Indeed \operatorname{str}(a b)=$ $(a, b)=(-1)^{p(a) p(b)}(b, a)=(-1)^{p(a) p(b)} \operatorname{str}(b a)$.

(Invariance) Since

$$
\begin{array}{r}
{[b, a c]=b(a c)-(-1)^{p(b)(p(a)+p(c))}(a c) b=} \\
b(a c)-(-1)^{p(a) p(b)}(a b) c+ \\
(-1)^{p(a) p(b)}(a b) c-(-1)^{p(b)(p(a)+p(c))}(a c) b= \\
{[b, a] c+(-1)^{p(a) p(b)} a\left(b c-(-1)^{p(b) p(c)} c b\right)=} \\
{[b, a] c+(-1)^{p(a) p(b)} a[b, c],}
\end{array}
$$

using 2) we have

$$
\begin{array}{r}
0=\operatorname{str}([b, a c])= \\
\operatorname{str}\left([b, a] c+(-1)^{p(b) p(a)} a[b, c]\right)= \\
-(-1)^{p(b) p(a)} \operatorname{str}([a, b] c)+(-1)^{p(b) p(a)} \operatorname{str}(a[b, c]) .
\end{array}
$$

Therefore $([a, b], c)=\operatorname{str}([a, b] c)=\operatorname{str}(a[b, c])=(a,[b, c])$. 


\subsection{Derivations}

Definition 6.14. Let $A$ be a superalgebra. A derivation of parity $s \in \mathbb{Z}_{2}$ is an endomorphism $D \in \operatorname{End}_{s}(A)$ with the property

$$
D(a b)=D(a) b+(-1)^{p(a) s} a D(b) .
$$

We denote by $\operatorname{der}_{s}(A) \subseteq \operatorname{End}(A)$ the space of all derivations of parity $s$ and we define $\operatorname{der}(A)=\operatorname{der}(A)_{\overline{0}} \oplus \operatorname{der}(A)_{\overline{1}}$.

Remark 51. Let $D$ a derivation of $A$. Then $D(1)=0$.

Proof. $D(1)=D(1 \cdot 1)=D(1) \cdot 1+(-1)^{p(D) p(1)} 1 \cdot D(1)=2 D(1)$. Therefore $D(1)=0$.

Remark 52. The space $\operatorname{der}(A) \subseteq \operatorname{End}(A)$ is a subalgebra of the Lie superalgebra $l(A)$.

Proof. Let $D, \tilde{D}$ be to derivations of $\operatorname{der}(A)$. Then

$$
\begin{array}{r}
{[D, \tilde{D}](a b)=\left(D \tilde{D}-(-1)^{p(D) p(\tilde{D})} \tilde{D} D\right)(a b)=} \\
D\left(\tilde{D}(a) b+(-1)^{p(\tilde{D}) p(a)} a \tilde{D}(b)\right)- \\
(-1)^{p(D) p(\tilde{D})} \tilde{D}\left(D(a) b+(-1)^{p(D) p(a)} a D(b)\right)= \\
D \tilde{D}(a) b+(-1)^{(p(\tilde{D})+p(a)) p(D)} \tilde{D}(a) D(b)+ \\
(-1)^{p(\tilde{D}) p(a)} D(a) \tilde{D}(b)+(-1)^{p(\tilde{D}) p(a)}(-1)^{p(D) p(a)} a D \tilde{D}(b)- \\
(-1)^{p(D) p(\tilde{D})} \tilde{D} D(a) b-(-1)^{p(D) p(\tilde{D})}(-1)^{p(\tilde{D})(p(D)+p(a))} D(a) \tilde{D}(b)- \\
(-1)^{p(D) p(\tilde{D})}(-1)^{p(D) p(a)} \tilde{D}(a) D(b)- \\
(-1)^{p(D) p(\tilde{D})}(-1)^{p(D) p(a)}(-1)^{p(a) p(\tilde{D})} a \tilde{D} D(b)= \\
D \tilde{D}(a) b+(-1)^{p(\tilde{D}) p(a)}(-1)^{p(D) p(a)} a D \tilde{D}(b)- \\
(-1)^{p(D) p(\tilde{D})} \tilde{D} D(a) b-(-1)^{p(D) p(\tilde{D})}(-1)^{p(D) p(a)}(-1)^{p(a) p(\tilde{D})} a \tilde{D} D(b)= \\
\left(D \tilde{D}(a)-(-1)^{p(D) p(\tilde{D})} \tilde{D} D(a)\right)(b)+ \\
(-1)^{p(a)(p(D)+p(\tilde{D}))} a\left(D \tilde{D}(b)-(-1)^{p(D) p(\tilde{D})} \tilde{D} D(b)\right)= \\
{[D, \tilde{D}](a) b+(-1)^{p(a) p([D, \tilde{D}])} a[D, \tilde{D}](b) .}
\end{array}
$$


Hence $[D, \tilde{D}]$ is a derivation. Moreover, since $D_{1}, D_{2}, D_{3} \in \operatorname{der}(A) \subseteq \operatorname{End}(A)$,

$$
\left[D_{1}, D_{2}\right]=-(-1)^{p\left(D_{1}\right) p\left(D_{2}\right)}\left[D_{2}, D_{1}\right]
$$

and

$$
\left[D_{1},\left[D_{2}, D_{3}\right]\right]=\left[\left[D_{1}, D_{2}\right], D_{3}\right]+(-1)^{p\left(D_{1}\right) p\left(D_{2}\right)}\left[D_{2},\left[D_{1}, D_{3}\right]\right]
$$

Example 6.4. Let $\mathfrak{g}$ be a Lie superalgebra. Then the map

$$
a d_{a}: \mathfrak{g} \rightarrow \mathfrak{g}, b \mapsto[a, b]
$$

where $a \in \mathfrak{g}$, is a derivation of $\mathfrak{g}$.

Proof. $\operatorname{ad}_{a}([b, c])=[a,[b, c]]=[[a, b], c]+(-1)^{p(a) p(b)}[b,[a, c]]=\left[a d_{a}(b), c\right]+$ $(-1)^{p\left(a d_{a}\right) p(b)}\left[b, a d_{a}(c)\right]$.

The Jacobi identity hence means that, for $a \in \mathfrak{g}, a d_{a}$ is a derivation of the Lie superalgebra $\mathfrak{g}$.

Remark 53. The space of the derivations of $\mathfrak{g}$ of the form $a d_{a}$ is an ideal of $\operatorname{der}(\mathfrak{g})$.

Proof. Since $\left[D, a d_{a}\right](b)=D([a, b])-(-1)^{p(D) p(a)}[a, D(b)]=[D(a), b]+$ $(-1)^{p(a) p(D)}[a, D(b)]-(-1)^{p(D) p(a)}[a, D(b)]=[D(a), b],\left[D, a d_{a}\right]=a d_{D(a)}$, i.e. the space of the derivations of $\mathfrak{g}$ of the form $a d_{a}$ is an ideal of $\operatorname{der}(\mathfrak{g})$.

\subsection{Linear representations of Lie superalge- bras}

Definition 6.15. Let $V=V_{\overline{0}} \oplus V_{\overline{1}}$ be a $\mathbb{Z}_{2}$-graded linear space. A linear representation $\phi$ of a Lie superalgebra $\mathfrak{g}=\mathfrak{g}_{\overline{0}} \oplus \mathfrak{g}_{\overline{1}}$ is a homomorphism of Lie superalgebras $\phi: G \rightarrow l(V)$. 
In this case we often say that $V$ is a $\mathfrak{g}$-module and we write $x . v$ instead of $\phi(x)(v)$ with $x \in \mathfrak{g}$ and $v \in V$. By definition $\mathfrak{g}_{i} . V_{j} \subseteq V_{i+j}$ and $[x, y] . v=$ $\phi([x, y])(v)=[\phi(x), \phi(y)](v)=\phi(x) \phi(y)(v)-(-1)^{(\operatorname{deg} x)(\operatorname{deg} y)} \phi(y) \phi(x)(v)=$ $x \cdot(y \cdot v)-(-1)^{(\operatorname{deg} x)(\operatorname{deg} y)} y \cdot(x \cdot v)$.

Remark 54. The map ad $: \mathfrak{g} \rightarrow l(\mathfrak{g})$ with $(\operatorname{ad}(x))(y)=[x, y]$ is a linear representation of $\mathfrak{g}$ and it is called the adjoint representation.

Definition 6.16. A $\mathfrak{g}$-module $V$ is called irreducible if it has no nontrivial submodules.

\section{$6.6 \mathbb{Z}$-gradings}

Definition 6.17. A $\mathbb{Z}$-grading of a superalgebra $A$ is a decomposition of $A$ into a direct sum of finite dimensional $\mathbb{Z}_{2}$-graded subspaces $A=\oplus_{i \in \mathbb{Z}} A_{i}$ for which $A_{i} A_{j} \subseteq A_{i+j}$. A $\mathbb{Z}$-grading is said to be consistent if $A_{\overline{0}}=\oplus_{i \in \mathbb{Z}} A_{2 i}$ and $A_{\overline{1}}=\oplus_{i \in \mathbb{Z}} A_{2 i+1}$.

Remark 55. If $\mathfrak{g}$ is a $\mathbb{Z}$-graded Lie superalgebra, then $\mathfrak{g}_{0}$ is a Lie superalgebra, because $\left[\mathfrak{g}_{0}, \mathfrak{g}_{0}\right] \subseteq \mathfrak{g}_{0}$ and the anticommutativity and the Jacoby identity for $\mathfrak{g}_{0}$ follow from the anticommutativity and the Jacobi identity on $\mathfrak{g}$. Moreover, since $\left[\mathfrak{g}_{0}, \mathfrak{g}_{i}\right] \subseteq \mathfrak{g}_{i}$, the restriction of the adjoint representation to $\mathfrak{g}_{0}$ induces linear representations of $\mathfrak{g}_{0}$ on the subspaces $\mathfrak{g}_{i}$.

Remark 56. We point out that Definition (6.17) can be, of course, given also for algebras, i.e. in the completely even case.

Definition 6.18. A $\mathbb{Z}$-graded Lie superalgebra $\mathfrak{g}=\oplus_{i \in \mathbb{Z}} \mathfrak{g}_{i}$ is called irreducible if the representation of $\mathfrak{g}_{0}$ on $\mathfrak{g}_{-1}$ is irreducible.

Definition 6.19. A $\mathbb{Z}$-graded Lie superalgebra $\mathfrak{g}=\bigoplus_{i \in \mathbb{Z}} \mathfrak{g}_{i}$ is called transitive if $\left[a, \mathfrak{g}_{-1}\right]=0$ for $a \in \mathfrak{g}_{i}, i \geq 0$, implies that $a=0$.

Example 6.5. Let $V=V_{\overline{0}} \oplus V_{\overline{1}}$ be a $\mathbb{Z}_{2}$-graded space. Then $l(V)$ has a consistent $\mathbb{Z}$-grading $l(V)=l_{-1} \oplus l(V)_{\overline{0}} \oplus l_{1}$ where, with respect to an 
homogeneous basis of $V, l_{-1}$ consists of matrices of the form

$$
\left(\begin{array}{ll}
0 & 0 \\
C & 0
\end{array}\right)
$$

and $l_{1}$ consists of matrices of the form

$$
\left(\begin{array}{ll}
0 & B \\
0 & 0
\end{array}\right) .
$$

\subsection{Criteria of simplicity}

Proposition 6.7.1. Let $\mathfrak{g}=\mathfrak{g}_{\overline{0}} \oplus \mathfrak{g}_{\overline{1}}$ be a Lie superalgebra. If the representation of $\mathfrak{g}_{\overline{0}}$ on $\mathfrak{g}_{\overline{1}}$ is faithful and irreducible and $\left[\mathfrak{g}_{\overline{1}}, \mathfrak{g}_{\overline{1}}\right]=\mathfrak{g}_{\overline{0}}$, then $\mathfrak{g}$ is simple

Proof. Let $I=I_{\overline{0}} \oplus I_{\overline{1}}$ be a nonzero ideal of $\mathfrak{g}$. $I_{\overline{1}}$ is a submodule of $\mathfrak{g}_{\overline{1}}$ because for $x \in \mathfrak{g}_{\overline{0}}$ and $y \in I_{\overline{1}},[x, y]$ lies in $I$ since $I$ is an ideal, and it is odd hence lies in $I_{\overline{1}}$. Since $\mathfrak{g}_{\overline{1}}$ is an irreducible $\mathfrak{g}_{\overline{0}}$-module, $I_{\overline{1}}$ is either 0 or $\mathfrak{g}_{\overline{1}}$. If $I_{\overline{1}}=0$, for all $x \in I_{\overline{0}}$ and $y \in \mathfrak{g}_{\overline{1}}[x, y]=0$ and, since the representation of $\mathfrak{g}_{\overline{0}}$ on $\mathfrak{g}_{\overline{1}}$ is faithful, $I_{\overline{0}}=0$, i.e. $I=I_{\overline{0}} \oplus I_{\overline{1}}=0$ which is against our hypothesis. Hence $I_{\overline{1}}=\mathfrak{g}_{\overline{1}}$. On the other hand $\mathfrak{g}_{\overline{0}}=\left[\mathfrak{g}_{\overline{1}}, \mathfrak{g}_{\overline{1}}\right]=\left[I_{\overline{1}}, I_{\overline{1}}\right] \subseteq I_{\overline{0}}$, i.e. $I_{\overline{0}}=\mathfrak{g}_{\overline{0}}$ and, consequently, $I=\mathfrak{g}$.

Proposition 6.7.2. Let $\mathfrak{g}=\oplus_{i \geq-1} \mathfrak{g}_{i}$ be a $\mathbb{Z}$-graded Lie superalgebra. If $\mathfrak{g}$ is transitive and irreducible, $\left[\mathfrak{g}_{-1}, \mathfrak{g}_{1}\right]=\mathfrak{g}_{0},\left[\mathfrak{g}_{0}, \mathfrak{g}_{1}\right]=\mathfrak{g}_{1}$ and $\mathfrak{g}_{i}=\mathfrak{g}_{1}^{i}$ for $i>0$, then $\mathfrak{g}$ is simple.

Proof. Let $I=\oplus_{i \geq-1} I_{i}$ be a nonzero ideal of $\mathfrak{g}$. $I_{-1}$ is a $\mathfrak{g}_{0}$-submodule of $\mathfrak{g}_{-1}$ because for all $x \in \mathfrak{g}_{0}$ and $y \in I_{-1},[x, y]$ lies in $I$ since $I$ is an ideal $y \in I$ and lies in $I_{-1}$. Since $\mathfrak{g}$ is irreducible, the $\mathfrak{g}_{0}$-module $\mathfrak{g}_{-1}$ is irreducible, i.e. either $I_{-1}=0$ or $I_{-1}=\mathfrak{g}_{-1}$. If $I_{-1}=0$, for all $x \in I_{0}$ and $y \in \mathfrak{g}_{-1}[x, y]=0$ because it lies in $I_{-1}$, then $x=0$ by transitivity, i.e. $I_{0}=0$. Repeating by induction on $i$ the same argument for $x \in I_{i}$ for all $i>0$ we obtain $I_{i}=0$ for all $i$, i.e. $I=0$. Hence $I_{-1}=\mathfrak{g}_{-1}$. It follows that $\mathfrak{g}_{0}=\left[\mathfrak{g}_{-1}, \mathfrak{g}_{1}\right]=\left[I_{-1}, \mathfrak{g}_{1}\right] \subseteq I_{0}$, 
i.e. $\quad I_{0}=\mathfrak{g}_{0}$, and $\mathfrak{g}_{1}=\left[\mathfrak{g}_{0}, \mathfrak{g}_{1}\right]=\left[I_{0}, \mathfrak{g}_{1}\right] \subseteq I_{1}$, i.e. $I_{1}=\mathfrak{g}_{1}$. Moreover $\mathfrak{g}_{i}=\mathfrak{g}_{1}^{i} \subseteq I_{1}^{i} \subseteq I_{i}$, i.e. $I_{i}=\mathfrak{g}_{i}$ for all $i>0$ and $I=\mathfrak{g}$.

Remark 57. Proposition (6.7.2) can be given also for a Lie algebra $\mathfrak{g}$, i.e. when $\mathfrak{g}$ is a completely even Lie superalgebra, with the additional request of $[\mathfrak{g}, \mathfrak{g}] \neq 0$. 


\section{Chapter 7}

\section{Examples}

\subsection{Outer tensor product}

In Example (1.18), we considered the tensor product of two representations of a Lie algebra $L$ and we defined on it a structure of an $L$-module. This construction does not preserve the irreducibility, namely, the tensor product of two irreducible $L$-modules is, in general, not irreducible.

Example 7.1. Let us consider the standard representation of $\mathfrak{s l}_{2}(\mathbb{F})$ and its adjoint representation. Then the $\mathfrak{s l}_{2}(\mathbb{F})$-module $V=\mathbb{F}^{2} \otimes \mathfrak{s l}_{2}(\mathbb{F})$ is not irreducible.

Proof. We already showed that $\mathbb{F}^{2}$ and $\mathfrak{s l}_{2}(\mathbb{F})$ are irreducible $\mathfrak{s l}_{2}(\mathbb{F})$-modules of dimension two and three respectively. Let $\left\{e_{1}, e_{2}\right\}$ be the canonical basis of $\mathbb{F}^{2}$ and let $\{x, y, h\}$ be the standard basis of $\mathfrak{s l}_{2}(\mathbb{F})$. We showed that $e_{1}$ and $x$ are maximal vectors in $\mathbb{F}^{2}$ and $\mathfrak{s l}_{2}(\mathbb{F})$, respectively. By Example (1.18) it thus follows that the vector

$$
v_{1}^{+}=e_{1} \otimes x
$$

is a maximal vector, indeed

$$
x .\left(e_{1} \otimes x\right)=\left(x e_{1}\right) \otimes x+e_{1} \otimes[x, x]=0
$$


besides,

$$
h .\left(e_{1} \otimes e\right)=\left(h e_{1}\right) \otimes x+e_{1} \otimes[h, x]=e_{1} \otimes x+2 e_{1} \otimes x=3 e_{1} \otimes x=3 v_{1}^{+} .
$$

This means that $v_{1}^{+}=e_{1} \otimes x$ is a maxmal vector of weight 3 in $V$, hence it generates an irreducible $\mathfrak{s l}_{2}(\mathbb{F})$-submodule of dimension 4 . This shows that $V$, which has dimension 6 , is not irreducible. Now note that $v_{2}^{+}=2 e_{2} \otimes x+e_{1} \otimes h$ is also a maximal vector in $V$, indeed:

$$
x .\left(2 e_{2} \otimes x+e_{1} \otimes h\right)=2\left(x e_{2}\right) \otimes x+e_{1} \otimes[x, h]=0
$$

and

$$
\begin{array}{r}
h .\left(2 e_{2} \otimes x+e_{1} \otimes h\right)=-2 e_{2} \otimes x+4 e_{2} \otimes x+e_{1} \otimes h= \\
2 e_{2} \otimes x+e_{1} \otimes h=v_{2}^{+} .
\end{array}
$$

It follows that $v_{2}^{+}$generates an irreducible $\mathfrak{s l}_{2}(\mathbb{F})$-module of $V$ of dimension 2. Therefore, by Corollary (5.1.2), $V \cong V(3) \oplus V(1)$.

We shall now introduce the concept of outer tensor product of two modules.

Definition 7.1. Let $L, K$ be Lie algebras, let $V$ be an $L$-module and let $W$ be a $K$-module. Then we can define a linear action of $L \oplus K$ on $V \otimes W$ as follows:

$$
(x+y) \cdot(v \otimes w)=(x \cdot v) \otimes w+v \otimes(y \cdot w)
$$

where $x \in L, y \in K, v \in V, w \in W$, and we extend this definition on $V \otimes W$ by linearity.

Remark 58. The linear space $V \otimes W$ with the action of $L \oplus K$ described in $\operatorname{Definition}(7.1)$ is an $(L \oplus K)$-module.

Proof. By construction we need to prove that:

$$
([x+y, \tilde{x}+\tilde{y}]) \cdot(v \otimes w)=(x+y) \cdot(\tilde{x}+\tilde{y}) \cdot(v \otimes w)-(\tilde{x}+\tilde{y}) \cdot(x+y) \cdot(v \otimes w) .
$$


We have $([x+y, \tilde{x}+\tilde{y}]) \cdot(v \otimes w)=([x, \tilde{x}]+[y, \tilde{y}]) \cdot(v \otimes w)=([x, \tilde{x}] \cdot v) \otimes$ $w+v \otimes([y, \tilde{y}] . w)=(x . \tilde{x} \cdot v-\tilde{x} \cdot x \cdot v) \otimes w+v \otimes(y \cdot \tilde{y} \cdot w-\tilde{y} \cdot y \cdot w)=x \cdot \tilde{x} \cdot v \otimes$ $w-\tilde{x} . x . v \otimes w+v \otimes y . \tilde{y} . w-v \otimes \tilde{y} . y . w$ where we used the structure of $L$ module and $K$-module on $V$ and $W$, respectively, and the linearity of the tensor product. On the other hand using the definition of the action and the linearity of the tensor product, the right hand side term of (7.3) is equal to $x . \tilde{x} . v \otimes w+\tilde{x} . v \otimes y . w+x . v \otimes \tilde{y} . w+v \otimes y . \tilde{y} . w-\tilde{x} . x . v \otimes w-x . v \otimes \tilde{y} . w-\tilde{x} . v \otimes$ $y \cdot w-v \otimes \tilde{y} . y \cdot w=x . \tilde{x} \cdot v \otimes w-\tilde{x} \cdot x \cdot v \otimes w+v \otimes y . \tilde{y} \cdot w-v \otimes \tilde{y} \cdot y \cdot w$.

Notation 1. Given an $L$-module $V$ and a $K$-module $W$, we will denote by $V \otimes W$ the tensor product $V \otimes W$ with the $L \oplus K$-module structure given in Definition (7.1). We will call $V \otimes W$ the outer tensor product of $V$ and $W$.

Proposition 7.1.1. Let $V$ be an irreducible L-module of finite dimension and let $W$ be an irreducible $K$-module of finite dimension. Then $V \otimes W$ is an irreducible $L \oplus K$-module.

Proof. Let $S$ be a nonzero $L \oplus K$-submodule of $V \otimes W$ and let $z=\sum_{i=1}^{l} v_{i} \otimes w_{i}$ with $v_{i} \in V$ and $w_{i} \in W$, be a nonzero element of $S$. Let us consider the set $\left\{v_{i_{1}}, \ldots, v_{i_{m}}\right\}$ of elements among the $v_{i}$ 's such that:

1. the $v_{i_{k}}$ 's are linearly independent,

2. every element $v_{i}$ is a linear combination of the $v_{i_{k}}$ 's.

Then we can write $z=\sum_{k=1}^{m} v_{i_{k}} \otimes \tilde{w}_{i_{k}}$ for some $\tilde{w}_{i_{k}} \in W$. Since the projection map $p_{V}: V \otimes W \rightarrow V$ is a homomorphism of $L$-modules, $p_{V}(S)$ is an $L$ submodule of $V$ and it is nonzero because $p_{V}(z)=\sum_{k=1}^{m} v_{i_{k}} \neq 0$. Therefore $p_{V}(S)=V$ due to the irreducibility of $V$. On the other hand we can repeat the same argument for $W$ considering the set $\left\{w_{j_{1}}, \ldots, w_{j_{n}}\right\}$ of the elements among the $w_{i}$ 's with the same properties as above. Hence $p_{W}(S)=W$. Let now $\mathcal{B}=\left\{v_{1}, \ldots v_{s}\right\}$ be a basis of $V$ and $\mathcal{B}^{\prime}=\left\{w_{1}, \ldots, w_{r}\right\}$ be a basis of $W$. Since $p_{V}(S)=V$, for every $v_{k} \in \mathcal{B}$, there exists some $\tilde{z} \in S$ such that $v_{k}=$ $p_{V}(\tilde{z})$. We can write $\tilde{z}=\sum_{i=1}^{s} \sum_{j=1}^{r} a_{i j} v_{i} \otimes w_{j}=\sum_{i=1}^{s} v_{i} \otimes\left(\sum_{j=1}^{r} a_{i j} w_{j}\right)=$ $\sum_{i=1}^{s} v_{i} \otimes \tilde{w}_{i}$ where we set $\tilde{w}_{i}=\sum_{j=1}^{r} a_{i j} w_{j}$, hence $v_{k}=p_{V}(\tilde{z})=\sum_{i=1}^{s} v_{i}$, 
i.e. we must have $\tilde{w}_{i}=0$ for $i \neq k$ and $\tilde{w}_{k} \neq 0$ because $\tilde{z} \neq 0$. It follows that $\tilde{z}=v_{k} \otimes \tilde{w}_{k}$. Moreover $p_{W}(\mathfrak{U}(K) . \tilde{z})=W$ because $p_{W}(\tilde{z}) \neq 0$ and $W$ is an irreducible $K$-module, therefore, for $w_{j} \in \mathcal{B}^{\prime}$, there exists some element $\bar{z} \in \mathfrak{U}(K)$. z such that $p_{W}(\bar{z})=w_{j}$ hence for every $k \in\{1, \ldots, s\}$ and $j \in\{1, \ldots, r\}$ there exists an element of $S$ of the form $v_{k} \otimes w_{j}$. It follows that $S=V \otimes W$.

\subsection{The action of $\mathfrak{g l}_{n}(\mathbb{F})$ on $\left(\mathbb{F}^{n}\right)^{*}$}

Let us consider the canonical basis $\left\{e_{1}, \ldots, e_{n}\right\}$ of $\mathbb{F}^{n}$ and its dual basis $\left\{\varphi_{e_{1}}, \ldots, \varphi_{e_{n}}\right\}$ of $\left(\mathbb{F}^{n}\right)^{*}$, i.e. $\varphi_{e_{i}}\left(e_{j}\right)=\delta_{i j}$. We can identify every vector $\varphi_{e_{i}}$ with the row vector $e_{i}^{*}=(0, \ldots, 0,1,0, \ldots, 0)$ with 1 in the $i$-th position. The vector space $\mathbb{F}^{n}$ is a $\mathfrak{g l}_{n}(\mathbb{F})$-module with the standard action given by matrix multiplication, hence $\left(\mathbb{F}^{n}\right)^{*}$ is a $\mathfrak{g l}_{n}(\mathbb{F})$-module with the following action:

$$
(X . \varphi)(v)=-\varphi(X v)
$$

where $X \in \mathfrak{g l}_{n}(\mathbb{F}), \varphi \in\left(\mathbb{F}^{n}\right)^{*}$ and $v \in \mathbb{F}^{n}$. Note that $\left(X . \varphi_{e_{i}}\right)\left(e_{j}\right)=$ $-\varphi_{e_{i}}\left(X e_{j}\right)=-\varphi_{e_{i}}\left(\sum_{k} X_{k j} e_{k}\right)=-X_{i j}$, i.e.

$$
X . \varphi_{e_{i}}=-\sum_{j} X_{i j} \varphi_{e_{j}} .
$$

According to the identification of $\varphi_{e_{j}}$ with $e_{j}^{*}$, we identify $X . \varphi_{e_{i}}$ with $X . e_{i}^{*}$ and $-\sum_{j} X_{i j} \varphi_{e_{j}}$ with $-\sum_{j} X_{i j} e_{j}^{*}$ thus, according to these notations, the action is the following:

$$
X . e_{i}^{*}=-\sum_{j} X_{i j} e_{j}^{*}=-e_{i}^{*} X
$$

\subsection{Example 1: the Lie superalgebra $l(m, n)$}

Let $V=V_{\overline{0}} \oplus V_{\overline{1}}$ be a $\mathbb{Z}_{2}$-graded space with $\operatorname{dim} V_{\overline{0}}=m$ and $\operatorname{dim} V_{\overline{1}}=n$ and let $\mathcal{H}=\left\{v_{1}, \ldots, v_{m}, v_{m+1}, \ldots, v_{m+n}\right\}$ be a homogeneous basis of $V$ where $\left\{v_{1}, \ldots, v_{m}\right\}$ is a basis of $V_{\overline{0}}$ and $\left\{v_{m+1}, \ldots, v_{m+n}\right\}$ is a basis of $V_{\overline{1}}$. We already 
noticed in Example (6.5) that the Lie superalgebra $l(m, n)$ has a consistent $\mathbb{Z}$-grading $l(m, n)=l_{-1} \oplus l(m, n)_{\overline{0}} \oplus l_{1}$ where, with respect to $\mathcal{H}, l_{-1}$ consists of the matrices of the form

$$
\left(\begin{array}{ll}
0 & 0 \\
C & 0
\end{array}\right)
$$

$l(m, n)_{\overline{0}}$ consists of the matrices of the form

$$
\left(\begin{array}{ll}
A & 0 \\
0 & D
\end{array}\right)
$$

and $l_{1}$ consists of the matrices of the form

$$
\left(\begin{array}{ll}
0 & B \\
0 & 0
\end{array}\right)
$$

with $A \in M_{m, m}(\mathbb{F}), B \in M_{m, n}(\mathbb{F}), C \in M_{n, m}(\mathbb{F})$ and $D \in M_{n, n}(\mathbb{F})$. From now on we will identify the endomorphisms in $l_{-1}, l(m, n)_{\overline{0}}$ and $l_{1}$ with their matrices with respect to the homogeneous basis $\mathcal{H}$. Then

- $l(m, n)_{\overline{0}} \cong \mathfrak{g l}_{m}(\mathbb{F}) \oplus \mathfrak{g l}_{n}(\mathbb{F})$ as Lie algebras,

- $l_{-1} \cong \mathbb{F}^{n} \otimes\left(\mathbb{F}^{m}\right)^{*}$ as $l(m, n) \overline{0}^{-m o d u l e s}$,

- $l_{1} \cong \mathbb{F}^{m} \otimes\left(\mathbb{F}^{n}\right)^{*}$ as $l(m, n)_{\overline{0}}$-modules.

Notation 2 . We denote by $(A, D)$ the matrices in $l(m, n)_{\overline{0}}$, i.e. $l(m, n)_{\overline{0}}=$ $\left\{(A, D) \mid A \in \mathfrak{g l}_{m}(\mathbb{F}), D \in \mathfrak{g l}_{n}(\mathbb{F})\right\}$.

We recall that $l_{-1}$ is a $l(m, n)_{\overline{0}}$-module with respect to the adjoint action

$$
\left[\left(\begin{array}{cc}
A & 0 \\
0 & D
\end{array}\right),\left(\begin{array}{ll}
0 & 0 \\
C & 0
\end{array}\right)\right]=\left(\begin{array}{cc}
0 & 0 \\
D C-C A & 0
\end{array}\right) .
$$

Lemma 7.3.1. The Lie algebra $l(m, n)_{\overline{0}}$ is isomorphic to the Lie algebra $\mathfrak{g l}_{n}(\mathbb{F}) \oplus \mathfrak{g l}_{m}(\mathbb{F})$.

Proof. Let us consider the linear map:

$$
\begin{aligned}
\phi: \mathfrak{g l}_{n}(\mathbb{F}) \oplus \mathfrak{g l}_{m}(\mathbb{F}) & \rightarrow l(m, n)_{\overline{0}} \\
D+A & \mapsto(A, D) .
\end{aligned}
$$


The linear map $\phi$ is an isomorphism of linear spaces, moreover $\phi([D+A, \tilde{D}+$ $\tilde{A}])=\phi([D, \tilde{D}]+[A, \tilde{A}])=([A, \tilde{A}],[D, \tilde{D}])=(A \tilde{A}-\tilde{A} A, D \tilde{D}-\tilde{D} D)=$ $(A \tilde{A}, D \tilde{D})-(\tilde{A} A, \tilde{D} D)=[(A, D),(\tilde{A}, \tilde{D})]=[\phi(D+A), \phi(\tilde{D}+\tilde{A})]$ hence $\phi$ is an isomorphism of Lie algebras.

Lemma 7.3.2. The set of matrices $M_{n, m}(\mathbb{F})$ is an $l(m, n)_{\overline{0}}$-module with the following action:

$$
(A, D) \cdot C=D C-C A \text {. }
$$

Proof. The action is linear on $l(m, n)_{\overline{0}}$ and on $M_{n, m}(\mathbb{F})$ by the definition of the action. Moreover $[(A, D),(\tilde{A}, \tilde{D})] \cdot C=(A \tilde{A}-\tilde{A} A, D \tilde{D}-\tilde{D} D) \cdot C=$ $D \tilde{D} C-\tilde{D} D C-C A \tilde{A}+C \tilde{A} A$, and $(A, D) \cdot(\tilde{A}, \tilde{D}) \cdot C-(\tilde{A}, \tilde{D}) \cdot(A, D) \cdot C=$ $(A, D) \cdot(\tilde{D} C-C \tilde{A})-(\tilde{A}, \tilde{D}) \cdot(D C-C A)=D \tilde{D} C-D C \tilde{A}-\tilde{D} C A+C \tilde{A} A-$ $\tilde{D} D C+\tilde{D} C A+D C \tilde{A}-C A \tilde{A}=D \tilde{D} C+C \tilde{A} A-\tilde{D} D C-C A \tilde{A}$, hence $M_{n, m}(\mathbb{F})$ is an $l(m, n) \overline{0}$-module.

Remark 59. The $l(m, n)_{\overline{0}}$-modules $l_{-1}$ and $M_{n, m}(\mathbb{F})$ are isomorphic.

Proposition 7.3.3. The $l(m, n)_{\overline{0}}$-module $l_{-1}$ is isomorphic to the outer tensor product $\mathbb{F}^{n} \otimes\left(\mathbb{F}^{m}\right)^{*}$.

Proof. Let us denote by $e_{i j}$ the matrices with $\left(e_{i j}\right)_{r s}=\delta_{i r} \delta_{j s}$ and let us consider the map:

$$
\begin{aligned}
\phi: M_{n, m}(\mathbb{F}) & \rightarrow \mathbb{F}^{n} \otimes\left(\mathbb{F}^{m}\right)^{*} \\
e_{i j} & \mapsto e_{i} \otimes e_{j}^{*}
\end{aligned}
$$

extended on $M_{n, m}(\mathbb{F})$ by linearity. The linear map $\phi$ is an isomorphism of vector spaces. Note that $\phi\left(D e_{i j}\right)=\phi\left(\sum_{k} D_{k i} e_{k j}\right)=\sum_{k} D_{k i} \phi\left(e_{k j}\right)=$ $\sum_{k} D_{k i}\left(e_{k} \otimes e_{j}^{*}\right)=\left(\sum_{k} D_{k i} e_{k}\right) \otimes e_{j}^{*}=D e_{i} \otimes e_{j}^{*}$ and $-\phi\left(e_{i j} A\right)=-\phi\left(\sum_{k} A_{j k} e_{i k}\right)=$ $-\sum_{k} A_{j k} \phi\left(e_{i k}\right)=-\sum_{k} A_{j k}\left(e_{i} \otimes e_{k}^{*}\right)=e_{i} \otimes\left(-\sum_{k} A_{j k} e_{k}^{*}\right)=e_{i} \otimes\left(-e_{j}^{*} A\right)$. It follows that $\phi\left((A, D) . e_{i j}\right)=\phi\left(D e_{i j}-e_{i j} A\right)=D e_{i} \otimes e_{j}^{*}+e_{i} \otimes\left(-e_{j}^{*} A\right)=$ $D . e_{i} \otimes e_{j}^{*}+e_{i} \otimes A . e_{j}^{*}=(D+A) .\left(e_{i} \otimes e_{j}^{*}\right)=(A, D) . \phi\left(e_{i j}\right)$, hence $\phi$ is an isomorphism of $l(m, n)_{\overline{0}}$-modules.

By the same argument we can prove the following results: 
Lemma 7.3.4. The Lie algebra $l(m, n)_{\overline{0}}$ is isomorphic to the Lie algebra $\mathfrak{g l}_{m}(\mathbb{F}) \oplus \mathfrak{g l}_{n}(\mathbb{F})$

Lemma 7.3.5. The set of matrices $M_{m, n}(\mathbb{F})$ is an $l(m, n)_{\overline{0}}$-module with the following action:

$$
(A, D) \cdot B=A B-B D
$$

Remark 60. The $l(m, n)_{\overline{0}}$-modules $l_{1}$ and $M_{m, n}(\mathbb{F})$ are isomorphic.

Proposition 7.3.6. The $l(m, n) \overline{0}$-module $l_{1}$ is isomorphic to the outer tensor product $\mathbb{F}^{m} \otimes\left(\mathbb{F}^{n}\right)^{*}$.

\subsection{Example 2: the classical Lie superalgebra}

$$
A(m, n)
$$

Definition 7.2. We define

$$
\mathfrak{s l}(m, n)=\{a \in l(m, n) \mid \operatorname{str}(a)=0\}
$$

where $\operatorname{str}(a)$ is the supertrace of $a$.

Remark 61 . The subspace $\mathfrak{s l}(m, n)$ is an ideal of $l(m, n)$ of codimension 1 .

Proof. Thanks to Proposition (6.3.1(2)), $\operatorname{str}([a, b])=0$ for all $a, b \in l(m, n)$. In particular, if $a \in \mathfrak{s l}(m, n)$ and $b \in l(m, n), \operatorname{str}([a, b])=0$, i.e. $[a, b]$ lies in $\mathfrak{s l}(m, n)$. Finally the condition on the supertrace forces $\mathfrak{s l}(m, n)$ to have codimension 1 in $l(m, n)$.

Remark 62. The subspace $\mathfrak{s l}(m, n)$ inherits the $\mathbb{Z}$-grading $l_{-1} \oplus \mathfrak{s l}(m, n)_{\overline{0}} \oplus l_{1}$ from $l(m, n)$ where $l_{-1}$ consists of the matrices of the form

$$
\left(\begin{array}{ll}
0 & 0 \\
C & 0
\end{array}\right)
$$

$\mathfrak{s l}(m, n)_{\overline{0}}$ consists of the matrices of the form

$$
\left(\begin{array}{ll}
A & 0 \\
0 & D
\end{array}\right)
$$


with $\operatorname{tr}(A)=\operatorname{tr}(D)$, because of the condition on the supertrace, and $l_{1}$ consists of the matrices of the form

$$
\left(\begin{array}{ll}
0 & B \\
0 & 0
\end{array}\right)
$$

with $A \in M_{m, m}(\mathbb{F}), B \in M_{m, n}(\mathbb{F}), C \in M_{n, m}(\mathbb{F})$ and $D \in M_{n, n}(\mathbb{F})$.

Remark 63. If $m=n, \mathfrak{s l}(n, n)$ contains the one dimensional ideal consisting on the scalar matrices $\lambda 1_{2 n}$. On the contrary, if $m \neq n$, the unique scalar multiple of the identity in $\mathfrak{s l}(m, n)$ is 0 (since char $\mathbb{F}=0$ ).

Definition 7.3. We define

$$
\begin{array}{ll}
A(m, n)=\mathfrak{s l}(m+1, n+1), \quad \text { for } m \neq n, m \geq 0, n \geq 0 \\
A(n, n)=\mathfrak{s l}(n+1, n+1) /\left\langle 1_{2 n+2}\right\rangle, & n \geq 0
\end{array}
$$

Remark 64 . The $\mathbb{Z}$-grading of $\mathfrak{s l}(m+1, n+1)$ induces a $\mathbb{Z}$-grading of $A(m, n)$ of the form $A(m, n)=l_{-1} \oplus A(m, n)_{\overline{0}} \oplus l_{1}$.

Lemma 7.4.1. For $m \neq n$, the Lie algebra $A(m, n)_{\overline{0}}$ is isomorphic to $\mathfrak{s l}_{m+1}(\mathbb{F}) \oplus \mathfrak{s l}_{n+1}(\mathbb{F}) \oplus \mathbb{F}_{L}$ and the Lie algebra $A(n, n)_{\overline{0}}$ is isomorphic to $\mathfrak{s l}_{n+1}(\mathbb{F}) \oplus$ $\mathfrak{s l}_{n+1}(\mathbb{F})$.

Proof. According to notation 2 we denote the elements of $\mathfrak{s l}(m+1, n+1)_{\overline{0}}$ by $(A, D)$ where $A \in \mathfrak{g l}_{m+1}(\mathbb{F}), D \in \mathfrak{g l}_{n+1}(\mathbb{F})$ and $\operatorname{tr}(A)=\operatorname{tr}(D)$. We can write $(A, D) \in \mathfrak{s l}(m+1, n+1)_{\overline{0}}$ as follows:

$$
(A, D)=\left(A-\frac{\operatorname{tr}(A)}{m+1} 1_{m+1}, D-\frac{\operatorname{tr}(A)}{n+1} 1_{n+1}\right)+\left(\frac{\operatorname{tr}(A)}{m+1} 1_{m+1}, \frac{\operatorname{tr}(A)}{n+1} 1_{n+1}\right)
$$

Since $\operatorname{tr}\left(A-\frac{\operatorname{tr}(A)}{m+1} 1_{m+1}\right)=0$ and $\operatorname{tr}\left(D-\frac{\operatorname{tr}(A)}{n+1} 1_{n+1}\right)=0, A-\frac{\operatorname{tr}(A)}{m+1} 1_{m+1}$ and $D-$ $\frac{\operatorname{tr}(A)}{n+1} 1_{n+1}$ lie in $\mathfrak{s l}_{m+1}(\mathbb{F})$ and in $\mathfrak{s l}_{n+1}(\mathbb{F})$ respectively. Moreover every matrix of $\mathfrak{s l}_{m+1}(\mathbb{F})\left(\mathfrak{s l}_{n+1}(\mathbb{F})\right)$ can be written as a difference between a matrix of $\mathfrak{g l}_{m+1}(\mathbb{F})\left(\mathfrak{g l}_{n+1}(\mathbb{F})\right)$ and a diagonal matrix. On the other hand the set of the matrices of the form $\left(\frac{\operatorname{tr}(A)}{m+1} 1_{m+1}, \frac{\operatorname{tr}(A)}{n+1} 1_{n+1}\right)$ is a Lie algebra isomorphic to $\mathbb{F}_{L}$. Therefore, as in Lemma (7.3.1), we can say that $\mathfrak{s l}(m+1, n+1)_{\overline{0}} \cong \mathfrak{s l}_{m+1}(\mathbb{F}) \oplus$ 
$\mathfrak{s l}_{n+1}(\mathbb{F}) \oplus\left\{\left(\frac{\lambda}{m+1} 1_{m+1}, \frac{\lambda}{n+1} 1_{n+1}\right), \lambda \in \mathbb{F}\right\}_{L} \cong \mathfrak{s l}_{m+1}(\mathbb{F}) \oplus \mathfrak{s l}_{n+1}(\mathbb{F}) \oplus \mathbb{F}_{L}$. It follows that if $m \neq n A(m, n)_{\overline{0}} \cong \mathfrak{s l}_{m+1}(\mathbb{F}) \oplus \mathfrak{s l}_{n+1}(\mathbb{F}) \oplus \mathbb{F}_{L}$ and $A(n, n)_{\overline{0}}=$ $\mathfrak{s l}(m+1, n+1)_{\overline{0}} /\left\langle 1_{2 n+2}\right\rangle \cong\left(\mathfrak{s l}_{n+1}(\mathbb{F}) \oplus \mathfrak{s l}_{n+1}(\mathbb{F}) \oplus\left\{\left(\frac{\lambda}{n+1} 1_{n+1}, \frac{\lambda}{n+1} 1_{n+1}\right), \lambda \in\right.\right.$ $\mathbb{F}\}) /\left\langle 1_{2 n+2}\right\rangle \cong \mathfrak{s l}_{n+1}(\mathbb{F}) \oplus \mathfrak{s l}_{n+1}(\mathbb{F})$.

Proposition 7.4.2. The $\mathfrak{s l}(m+1, n+1) \overline{0}_{-}$-modules $l_{-1}$ and $\mathbb{F}^{n+1} \otimes\left(\mathbb{F}^{m+1}\right)^{*} \otimes \mathbb{F}$ are isomorphic. In particular, for $A(m, n), l_{-1} \cong \mathbb{F}^{n+1} \otimes\left(\mathbb{F}^{m+1}\right)^{*} \otimes \mathbb{F}$, if $m \neq n$, and $l_{-1} \cong \mathbb{F}^{n+1} \otimes\left(\mathbb{F}^{n+1}\right)^{*}$, if $m=n$.

Proof. Let us consider the case $m \neq n$. Given $(A, D) \in \mathfrak{s l}(m+1, n+1)_{\overline{0}}$ we set $\tilde{A}=A-\frac{\operatorname{tr}(A)}{m+1} 1_{m+1}$ and $\tilde{D}=D-\frac{\operatorname{tr}(A)}{n+1} 1_{n+1}$, thus we can write $(A, D)$ as follows:

$$
(A, D)=(\tilde{A}, \tilde{D})+\left(\frac{\operatorname{tr}(A)}{m+1} 1_{m+1}, \frac{\operatorname{tr}(A)}{n+1} 1_{n+1}\right) .
$$

The action of $\mathfrak{s l}(m+1, n+1)_{\overline{0}}$ on $l_{-1}$ is the following:

$$
\begin{array}{r}
\left.\left[\begin{array}{cc}
\tilde{A} & 0 \\
0 & \tilde{D}
\end{array}\right)+\left(\begin{array}{cc}
\frac{\operatorname{tr}(A)}{m+1} 1_{m+1} & 0 \\
0 & \frac{\operatorname{tr}(A)}{n+1} 1_{n+1}
\end{array}\right),\left(\begin{array}{cc}
0 & 0 \\
C & 0
\end{array}\right)\right]= \\
\left(\begin{array}{cc}
0 & 0 \\
\tilde{D} C-C \tilde{A} & 0
\end{array}\right)+\left(\begin{array}{cc}
0 & 0 \\
\frac{\operatorname{tr}(A)}{n+1} C-\frac{\operatorname{tr}(A)}{m+1} C & 0
\end{array}\right)= \\
\left(\begin{array}{ccc}
0 & 0 \\
\tilde{D} C-C \tilde{A}+\operatorname{tr}(A)\left(\frac{m-n}{(n+1)(m+1)}\right) C & 0
\end{array}\right) .
\end{array}
$$

The following action of $\mathfrak{s l}(m+1, n+1)_{\overline{0}}$ on $M_{n+1, m+1}(\mathbb{F})$

$$
(A, D) \cdot C=\tilde{D} C-C \tilde{A}+\operatorname{tr}(A)\left(\frac{m-n}{(n+1)(m+1)}\right) C
$$

turns $M_{n+1, m+1}(\mathbb{F})$ into a $\mathfrak{s l}(m+1, n+1)_{\overline{0}}$-module which is isomorphic to the $\mathfrak{s l}(m+1, n+1) \overline{0^{-}}$-modules $l_{-1}$ (the proofs are identical to the ones of Lemma (7.3.2) and Remark (59)). We now want to prove that the $\mathfrak{s l}(m+1, n+1)_{\overline{0}^{-}}$ modules $M_{n+1, m+1}(\mathbb{F})$ and $\mathbb{F}^{n+1} \otimes\left(\mathbb{F}^{m+1}\right)^{*} \otimes \mathbb{F}$ are isomorphic. Let us consider the map:

$$
\varphi: M_{n+1, m+1}(\mathbb{F}) \rightarrow \mathbb{F}^{n+1} \otimes\left(\mathbb{F}^{m+1}\right)^{*} \otimes \mathbb{F}
$$




$$
e_{i j} \quad \mapsto \quad e_{i} \otimes e_{j}^{*} \otimes 1
$$

and extend $\varphi$ on $M_{n+1, m+1}(\mathbb{F})$ by linearity.

The map $\varphi$ is an isomorphism of vector spaces and $\varphi$ is a homomorphism of $\mathfrak{s l}(m+1, n+1) \overline{0}$-modules, indeed

$$
\begin{array}{r}
(A, D) . e_{i j}=\tilde{D} e_{i j}-e_{i j} \tilde{A}+\operatorname{tr}(A)\left(\frac{m-n}{(n+1)(m+1)}\right) e_{i j} \mapsto \tilde{D} e_{i} \otimes e_{j}^{*} \otimes 1+ \\
-e_{i} \otimes e_{j}^{*} \tilde{A} \otimes 1+\operatorname{tr}(A)\left(\frac{m-n}{(n+1)(m+1)}\right) e_{i} \otimes e_{j}^{*} \otimes 1= \\
\tilde{D} e_{i} \otimes e_{j}^{*} \otimes 1-e_{i} \otimes e_{j}^{*} \tilde{A} \otimes 1+e_{i} \otimes e_{j}^{*} \otimes \operatorname{tr}(A)\left(\frac{m-n}{(n+1)(m+1)}\right)= \\
\left(\tilde{D}+\tilde{A}+\operatorname{tr}(A)\left(\frac{m-n}{(n+1)(m+1)}\right)\right) \cdot\left(e_{i} \otimes e_{j}^{*} \otimes 1\right)= \\
(A, D) \cdot\left(e_{i} \otimes e_{j}^{*} \otimes 1\right)=(A, D) \cdot \varphi\left(e_{i j}\right)
\end{array}
$$

where $D e_{i j} \mapsto \tilde{D} e_{i} \otimes e_{j}^{*} \otimes 1$ and $-e_{i j} \tilde{A} \mapsto-e_{i} \otimes e_{j}^{*} \tilde{A} \otimes 1$ by the same arguments as those used in the proof of Proposition (7.3.3). Hence $\varphi$ is an isomorphism of $\mathfrak{s l}(m+1, n+1) \overline{0}^{-}$-modules.

For the case $m=n$ the proof is the same of the one of Proposition (7.3.3).

With an identical proof the following proposition holds:

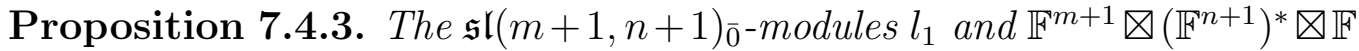
are isomorphic. In particular, for $A(m, n), l_{1} \cong \mathbb{F}^{m+1} \otimes\left(\mathbb{F}^{n+1}\right)^{*} \otimes \mathbb{F}$, if $m \neq n$, and $l_{1} \cong \mathbb{F}^{n+1} \otimes\left(\mathbb{F}^{n+1}\right)^{*}$, if $m=n$.

Remark $65 . l_{1}$ is an irreducible $A(m, n) \overline{0}^{- \text {-module. }}$

Proof. Since the Lie algebras $\mathfrak{s l}_{m+1}(\mathbb{F})$ and $\mathfrak{s l}_{n+1}(\mathbb{F})$ act transitively on $\mathbb{F}^{m+1}$ and on $\mathbb{F}^{n+1}$ respectively, $\mathbb{F}^{m+1}$ is an irreducible $\mathfrak{s l}_{m+1}(\mathbb{F})$-module and $\mathbb{F}^{n+1}$ is an irreducible $\mathfrak{s l}_{n+1}(\mathbb{F})$-module. Therefore $\left(\mathbb{F}^{n+1}\right)^{*}$ is also an irreducible $\mathfrak{s l}_{n+1}(\mathbb{F})$-module by Proposition (1.1.5). Hence, by Proposition (7.1.1), $l_{1}$ is an irreducible $A(n, n)_{\overline{0}}$-module. Moreover $\mathbb{F}$ is an irreducible $\mathbb{F}_{L}$-module hence, by Proposition (7.1.1), $l_{1}$ is an irreducible $A(m, n) \overline{0}^{-}$-module also for $m \neq n$. 
Lemma 7.4.4. The superalgebra $A(m, n)$ is transitive.

Proof. Let us consider a matrix $a \in \mathfrak{s l}(m+1, n+1)_{\overline{0}}, a=\left(\begin{array}{cc}A & 0 \\ 0 & D\end{array}\right)$ where $\operatorname{tr}(A)=\operatorname{tr}(D)$. We want to prove that $\left[a, l_{-1}\right]=0$ implies $a=0$, i.e. $\left[\left(\begin{array}{cc}A & 0 \\ 0 & D\end{array}\right),\left(\begin{array}{ll}0 & 0 \\ C & 0\end{array}\right)\right]=0$ for all $C \in M_{n, m}(\mathbb{F})$ implies $A=D=0$.

$$
0=\left[\left(\begin{array}{cc}
A & 0 \\
0 & D
\end{array}\right),\left(\begin{array}{cc}
0 & 0 \\
e_{i j} & 0
\end{array}\right)\right]=\left(\begin{array}{cc}
0 & 0 \\
D e_{i j}-e_{i j} A & 0
\end{array}\right),
$$

therefore $0=D e_{i j}-e_{i j} A$, i.e. $D e_{i j}=e_{i j} A$. Since $D e_{i j}=\sum_{k=1}^{n} D_{k i} e_{k j}$ and $e_{i j} A=\sum_{k=1}^{m} A_{j k} e_{i k}, D_{i i}=A_{j j}$ and $D_{k i}=0$ for $k \neq i, A_{j k}=0$ for $k \neq j$. Moreover this holds for all $i=1, \ldots, n$ and $j=1, \ldots, m$. Hence $a=\left(\begin{array}{cc}A & 0 \\ 0 & D\end{array}\right)$ is a scalar multiple of the identity, i.e. $a$ is 0 because the only scalar multiple of the identity in $A(m, n)$ is 0 .

Let us now consider a matrix $b \in l_{1}, b=\left(\begin{array}{cc}0 & B \\ 0 & 0\end{array}\right)$. We want to prove that $\left[b, l_{-1}\right]=0$ implies $b=0$, i.e. $\left[\left(\begin{array}{ll}0 & B \\ 0 & 0\end{array}\right),\left(\begin{array}{ll}0 & 0 \\ C & 0\end{array}\right)\right]=0$ for all $C \in M_{n, m}(\mathbb{F})$ implies $B=0$.

$$
0=\left[\left(\begin{array}{cc}
0 & B \\
0 & 0
\end{array}\right),\left(\begin{array}{cc}
0 & 0 \\
e_{i j} & 0
\end{array}\right)\right]=\left(\begin{array}{cc}
B e_{i j} & 0 \\
0 & e_{i j} B
\end{array}\right)
$$

therefore $0=B e_{i j}=\sum_{k=1}^{m} B_{k i} e_{k j}$, i.e. $B_{k i}=0$ for all $k=1, \ldots, m$. On the other hand we can choose $e_{i j}$ with $i=1, \ldots, n$, hence $B_{k i}=0$ for all $k=1, \ldots, m$ and for all $i=1, \ldots, n$, i.e. $B=0$.

Lemma 7.4.5. $\left[l_{-1}, l_{1}\right]=A(m, n)_{\overline{0}}$

Proof. $\left[l_{-1}, l_{1}\right] \subseteq A(m, n)_{\overline{0}}$ because $\left[\left(\begin{array}{cc}0 & 0 \\ C & 0\end{array}\right),\left(\begin{array}{cc}0 & B \\ 0 & 0\end{array}\right)\right]=\left(\begin{array}{cc}B C & 0 \\ 0 & C B\end{array}\right)$ and $\operatorname{tr}(B C)=\operatorname{tr}(C B)$. So we want to prove $A(m, n)_{\overline{0}} \subseteq\left[l_{-1}, l_{1}\right]$. Since $\left[\left(\begin{array}{cc}0 & 0 \\ e_{i j} & 0\end{array}\right),\left(\begin{array}{cc}0 & e_{l k} \\ 0 & 0\end{array}\right)\right]=\left(\begin{array}{cc}\delta_{k i} e_{l j} & 0 \\ 0 & \delta_{j l} e_{i k}\end{array}\right)$, if $i \neq k$ and $j=l$ we get all 
the matrices of the form $\left(\begin{array}{cc}0 & 0 \\ 0 & e_{i k}\end{array}\right)$, if $k=i$ and $j \neq l$ we get $\left(\begin{array}{cc}e_{l j} & 0 \\ 0 & 0\end{array}\right)$, i.e. $\left[l_{-1}, l_{1}\right]$ contains all the elements out of the diagonal of matrices in $A(m, n)_{\overline{0}}$. If $k=i$ and $j=l$ we have $\left(\begin{array}{cc}e_{j j} & 0 \\ 0 & e_{i i}\end{array}\right)$ whose linear combinations give all the diagonal elements of a matrix with 0 -supertrace.

Definition 7.4. A finite dimensional Lie superalgebra $\mathfrak{g}=\mathfrak{g}_{\overline{0}} \oplus \mathfrak{g}_{\overline{1}}$ is called classical if it is simple and the representation of $\mathfrak{g}_{\overline{0}}$ on $\mathfrak{g}_{\overline{1}}$ is completely reducible.

Proposition 7.4.6. The Lie superalgebra $A(m, n)$ is classical.

Proof. Since $\mathbb{F}^{n+1}$ is an irreducible $\mathfrak{s l}_{n+1}(\mathbb{F})$-module, $\left(\mathbb{F}^{m+1}\right)^{*}$ is an irreducible $\mathfrak{s l}_{m+1}(\mathbb{F})$-module and $\mathbb{F}$ is an irreducible $\mathbb{F}_{L}$-module, by Proposition (7.1.1), $\mathbb{F}^{n+1} \otimes\left(\mathbb{F}^{m+1}\right)^{*} \otimes \mathbb{F} \cong l_{-1}$, if $m \neq n$, and $\mathbb{F}^{n+1} \otimes\left(\mathbb{F}^{n+1}\right)^{*} \cong l_{-1}$, if $m=$

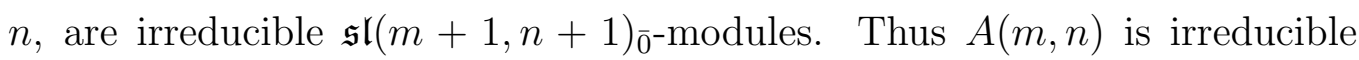
because $l_{-1}$ is an irreducible $A(m, n)_{\overline{0}}$-module. By Lemma (7.4.4), $A(m, n)$ is transitive, by Lemma (7.4.5) $\left[l_{-1}, l_{1}\right]=A(m, n)_{\overline{0}}$ and by Remark (65) $\left[A(m, n)_{\overline{0}}, l_{1}\right]=l_{1}$. Hence by Proposition $(6.7 .2) A(m, n)$ is simple. Moreover $A(m, n)_{\overline{1}}=l_{-1} \oplus l_{1} \cong \mathbb{F}^{n+1} \otimes\left(\mathbb{F}^{m+1}\right)^{*} \otimes \mathbb{F} \oplus \mathbb{F}^{m+1} \otimes\left(\mathbb{F}^{n+1}\right)^{*} \otimes \mathbb{F}$, if $m \neq n$, and $A(n, n)_{\overline{1}}=l_{-1} \oplus l_{1} \cong \mathbb{F}^{n+1} \otimes\left(\mathbb{F}^{n+1}\right)^{*} \oplus \mathbb{F}^{n+1} \otimes\left(\mathbb{F}^{n+1}\right)^{*}$, if $m=n$, are completely reducible $A(m, n)_{\overline{0}}$-modules. It follows that $A(m, n)$ is a classical Lie superalgebra.

\subsection{Example 3: the Lie algebra $W(m, 0)$}

Let us consider the Lie algebra $\mathbb{F}\left[x_{1}, \ldots, x_{m}\right]$ of polynomials in $m$ symbols $x_{1}, \ldots, x_{m}$.

Remark 66. By definition of derivation of an algebra, if $D$ is a derivation of $A=\mathbb{F}\left[x_{1}, \ldots, x_{m}\right]$, then $D(1)=0$. It follows that the images $D\left(x_{i}\right)$ define a derivation of $A$ completely, due to the linearity of $D$ and the Leibniz rule 
(1.5). In particular, the maps

$$
\begin{gathered}
\frac{\partial}{\partial x_{i}}: A \rightarrow A \\
\frac{\partial}{\partial x_{i}}\left(x_{j}\right)=\delta_{i j}
\end{gathered}
$$

for $i, j=1, \ldots, m$ extend uniquely to derivations of $A$.

Notation 3. We will denote the Lie algebra of derivations $\operatorname{der}\left(\mathbb{F}\left[x_{1}, \ldots, x_{m}\right]\right)$ by $W(m, 0)$.

\section{Proposition 7.5.1.}

$$
W(m, 0)=\left\{X=\sum_{i=1}^{m} P_{i} \frac{\partial}{\partial x_{i}} \mid P_{i} \in \mathbb{F}\left[x_{1}, \ldots, x_{m}\right]\right\}
$$

Proof. The vector fields $X=\sum_{i=1}^{m} P_{i} \frac{\partial}{\partial x_{i}}$ are derivations because $\frac{\partial}{\partial x_{i}}$ is a derivation for every $i$. Let now $D \in W(m, 0)$. Then $D\left(x_{i}\right)=P_{i}$ for some $P_{i} \in \mathbb{F}\left[x_{1}, \ldots, x_{m}\right]$. It follows that $D=\sum_{i=1}^{m} P_{i} \frac{\partial}{\partial x_{i}}$.

Remark 67 . If we set $\operatorname{deg} x_{i}=1$ for $i=1, \ldots, m$, we obtain a $\mathbb{Z}$-grading of $\mathbb{F}\left[x_{1}, \ldots, x_{m}\right]$

$$
\mathbb{F}\left[x_{1}, \ldots, x_{m}\right]=\oplus_{k \geq 0} \mathbb{F}\left[x_{1}, \ldots, x_{m}\right]_{k}
$$

where, by an abuse of notation, we denote by $\mathbb{F}\left[x_{1}, \ldots, x_{m}\right]_{k}$ the vector space $\left\langle x_{i_{1}} \cdots x_{i_{k}} \frac{\partial}{\partial x_{j}}\right\rangle$. This $\mathbb{Z}$-grading induces a $\mathbb{Z}$-grading of $W(m, 0)$ :

$$
W(m, 0)=\oplus_{k \geq-1} W(m, 0)_{k}
$$

with

$$
\begin{array}{r}
W(m, 0)_{k}=\left\{D \in W(m, 0) \mid D\left(\mathbb{F}\left[x_{1}, \ldots, x_{m}\right]_{i}\right) \subseteq \mathbb{F}\left[x_{1}, \ldots, x_{m}\right]_{i+k}\right\}= \\
\left\{\sum_{i} P_{i} \frac{\partial}{\partial x_{i}} \mid P_{i} \in \mathbb{F}\left[x_{1}, \ldots, x_{m}\right]_{k+1}, i=1, \ldots, n\right\} .
\end{array}
$$

Note that $W(m, 0)_{-1}=\left\langle\frac{\partial}{\partial x_{1}}, \ldots, \frac{\partial}{\partial x_{m}}\right\rangle$ and that $\left[\frac{\partial}{\partial x_{i}}, \frac{\partial}{\partial x_{j}}\right] \in W(m, 0)_{-2}=$ 0 consistently with

$$
\frac{\partial}{\partial x_{i}} \frac{\partial}{\partial x_{j}}=\frac{\partial}{\partial x_{j}} \frac{\partial}{\partial x_{i}}
$$


Proposition 7.5.2. 1. $W(m, 0)=\oplus_{k \geq-1} W(m, 0)_{k}$ is transitive.

2. The Lie algebras $W(m, 0)_{0}$ and $\mathfrak{g l}_{m}(\mathbb{F})$ are isomorphic.

3. The $W(m, 0)_{0}$-modules $W(m, 0)_{-1}$ and $\left(\mathbb{F}^{m}\right)^{*}$ are isomorphic.

4. $W(m, 0)_{k}=W(m, 0)_{1}^{k}$ for $k \geq 1$ and $m \geq 2$.

5. $W(m, 0)$ is simple for $m \geq 2$.

Proof. 1) Let us consider $a \in W(m, 0)_{\geq 0}$ such that $\left[a, W(m, 0)_{-1}\right]=0$. We want to prove that $a=0$. We can assume $a \in W(m, 0)_{k}$, i.e. $a=$ $\sum_{i=1}^{m} P_{i} \frac{\partial}{\partial x_{i}}$ with $P_{i} \in \mathbb{F}\left[x_{1}, \ldots, x_{m}\right]_{k+1}$, then $0=\left[a, W(m, 0)_{-1}\right]$ if and only if $0=\left[a, \frac{\partial}{\partial x_{j}}\right]=\sum_{i}\left[P_{i} \frac{\partial}{\partial x_{i}}, \frac{\partial}{\partial x_{j}}\right]=-\sum_{i}\left(\frac{\partial P_{i}}{\partial x_{j}}\right) \frac{\partial}{\partial x_{i}}$ for all $j=1, \ldots, m$. Therefore $\frac{\partial P_{i}}{\partial x_{j}}=0$ for all $i=1, \ldots, m$. Since this relation holds for every $j=1, \ldots, m, P_{i}$ is a scalar. On the other hand $P_{i} \in \mathbb{F}\left[x_{1}, \ldots, x_{m}\right]_{k+1}$ with $k \geq 0$, hence $P_{i}=0$ because the only scalar in $\mathbb{F}\left[x_{1}, \ldots, x_{m}\right]_{k+1}$ with $k \geq 0$ is 0 .

2) Since $\left[W(m, 0)_{0}, W(m, 0)_{0}\right] \subseteq W(m, 0)_{0}$ and $W(m, 0)$ is a Lie algebra, $W(m, 0)_{0}$ is a Lie algebra. $W(m, 0)_{0}=\left\{\sum_{i} P_{i} \frac{\partial}{\partial x_{i}} \mid P_{i} \in \mathbb{F}\left[x_{1}, \ldots, x_{m}\right]_{1}\right\}=$ $\left\langle x_{i} \frac{\partial}{\partial x_{j}} \mid i, j=1, \ldots, m\right\rangle$. Let us define the following linear map:

$$
\phi: \mathfrak{g l}_{m}(\mathbb{F}) \rightarrow W(m, 0)_{0}, e_{i j} \mapsto x_{i} \frac{\partial}{\partial x_{j}}
$$

Since the linear map $\phi$ is linear and surjective by construction and $\operatorname{dim} \mathfrak{g l}_{m}(\mathbb{F})=$ 
$\operatorname{dim} W(m, 0)_{0}, \phi$ is an isomorphism of vector spaces. Moreover

$$
\begin{array}{r}
\phi([A, B])=\phi\left(\left[\sum_{i, j=1}^{n} A_{i j} e_{i j}, \sum_{k, l=1}^{n} B_{k l} e_{k l}\right]\right)= \\
\sum_{i, j=1}^{n} \sum_{k, l=1}^{n} A_{i j} B_{k l} \phi\left(\left[e_{i j}, e_{k l}\right]\right)= \\
\sum_{i, j=1}^{n} \sum_{k, l=1}^{n} A_{i j} B_{k l} \phi\left(\delta_{j k} e_{i l}-\delta_{l i} e_{k j}\right)= \\
\sum_{i, j=1}^{n} \sum_{k, l=1}^{n} A_{i j} B_{k l} \delta_{j k} \phi\left(e_{i l}\right)-\sum_{i, j=1}^{n} \sum_{k, l=1}^{n} A_{i j} B_{k l} \delta_{l i} \phi\left(e_{k j}\right)= \\
\sum_{i, j=1}^{n} \sum_{l=1}^{n} A_{i j} B_{j l} x_{i} \frac{\partial}{\partial x_{l}}-\sum_{i, j=1}^{n} \sum_{k=1}^{n} A_{i j} B_{k i} x_{k} \frac{\partial}{\partial x_{j}}
\end{array}
$$

and

$$
\begin{array}{r}
{[\phi(A), \phi(B)]=\sum_{i, j=1}^{n} \sum_{k, l=1}^{n} A_{i j} B_{k l}\left[\phi\left(e_{i j}\right), \phi\left(e_{k l}\right)\right]=} \\
\sum_{i, j=1}^{n} \sum_{k, l=1}^{n} A_{i j} B_{k l}\left[x_{i} \frac{\partial}{\partial x_{j}}, x_{k} \frac{\partial}{\partial x_{l}}\right]= \\
\sum_{i, j=1}^{n} \sum_{k, l=1}^{n} A_{i j} B_{k l}\left(\delta_{j k} x_{i} \frac{\partial}{\partial x_{l}}-\delta_{l i} x_{k} \frac{\partial}{\partial x_{j}}\right)= \\
\sum_{i, j=1}^{n} \sum_{l=1}^{n} A_{i j} B_{j l} x_{i} \frac{\partial}{\partial x_{l}}-\sum_{i, j=1}^{n} \sum_{k=1}^{n} A_{i j} B_{k i} x_{k} \frac{\partial}{\partial x_{j}} .
\end{array}
$$

Therefore $\phi$ is an isomorphism of Lie algebras.

3) $W(m, 0)_{-1}$ is a $W(m, 0)_{0}$-module with respect to the restriction of the adjoint action,

$$
W(m, 0)_{-1}=\left\langle\frac{\partial}{\partial x_{1}}, \ldots, \frac{\partial}{\partial x_{n}}\right\rangle
$$

Let us define the following linear map:

$$
\psi:\left(\mathbb{F}^{m}\right)^{*} \rightarrow W(m, 0)_{-1}, e_{i}^{*} \mapsto \frac{\partial}{\partial x_{i}} .
$$

Since the map $\psi$ is linear, surjective and $\operatorname{dim}\left(\mathbb{F}^{m}\right)^{*}=\operatorname{dim} W(m, 0)_{-1}, \psi$ is an isomorphism of vector spaces. Moreover, thank to the isomorphism of Lie 
algebras defined in (7.22),

$$
\begin{array}{r}
\psi\left(\left(\sum_{i, j=1}^{n} A_{i j} x_{i} \frac{\partial}{\partial x_{j}}\right) \cdot\left(\sum_{k}^{n} b_{k} e_{k}^{*}\right)\right)=\psi\left(\left(\sum_{i, j=1}^{n} A_{i j} e_{i j}\right) \cdot\left(\sum_{k}^{n} b_{k} e_{k}^{*}\right)\right)= \\
\psi\left(-\sum_{i, j} \sum_{k} A_{i j} b_{k} \delta_{k i} e_{j}^{*}\right)=-\sum_{i, j} A_{i j} b_{i} \frac{\partial}{\partial x_{j}}
\end{array}
$$

and

$$
\begin{array}{r}
\left(\sum_{i, j=1}^{n} A_{i j} x_{i} \frac{\partial}{\partial x_{j}}\right) \cdot \psi\left(\sum_{k}^{n} b_{k} e_{k}^{*}\right)= \\
\left(\sum_{i, j=1}^{n} A_{i j} x_{i} \frac{\partial}{\partial x_{j}}\right) \cdot\left(\sum_{k}^{n} b_{k} \frac{\partial}{\partial x_{k}}\right)=\sum_{i, j=1}^{n} \sum_{k}^{n} A_{i j} b_{k}\left[x_{i} \frac{\partial}{\partial x_{j}}, \frac{\partial}{\partial x_{k}}\right]= \\
\sum_{i, j=1}^{n} \sum_{k}^{n} A_{i j} b_{k}\left(x_{i} \frac{\partial}{\partial x_{j}} \frac{\partial}{\partial x_{k}}-\delta_{i k} \frac{\partial}{\partial x_{j}}-x_{i} \frac{\partial}{\partial x_{k}} \frac{\partial}{\partial x_{i}}\right)= \\
-\sum_{i, j=1}^{n} A_{i j} b_{i} \frac{\partial}{\partial x_{j}}
\end{array}
$$

Therefore $\psi$ is a homomorphism of $W(m, 0)_{0}$-modules and an isomorphism of vector spaces. It follows that $\psi$ is an isomorphism of $W(m, 0)_{0}$-modules. 4) $W(m, 0)_{1}=\left\langle x_{i} x_{j} \frac{\partial}{\partial x_{k}}\right\rangle$. Let us prove that $W(m, 0)_{k}=W(m, 0)_{1}^{k}$ by induction on $k$. If $k=1$ there is nothing to prove. Let us suppose that $W(m, 0)_{k}=W(m, 0)_{1}^{k}$ and let us prove that $W(m, 0)_{k+1}=W(m, 0)_{1}^{k+1}$. $W(m, 0)_{1}^{k+1} \subseteq W(m, 0)_{k+1}$ because of the $\mathbb{Z}$-grading of $W(m, 0)$, so we only need to prove that $W(m, 0)_{k+1} \subseteq W(m, 0)_{1}^{k+1}$.

$$
\begin{array}{r}
{\left[P_{j} \frac{\partial}{\partial x_{j}}, x_{i_{1}} x_{i_{2}} \frac{\partial}{\partial x_{i_{3}}}\right]=} \\
\delta_{j i_{1}} P_{j} x_{i_{2}} \frac{\partial}{\partial x_{i_{3}}}+\delta_{j i_{2}} P_{j} x_{i_{1}} \frac{\partial}{\partial x_{i_{3}}}-x_{i_{1}} x_{i_{2}}\left(\frac{\partial P_{j}}{\partial x_{i_{3}}}\right) \frac{\partial}{\partial x_{j}}
\end{array}
$$

with $j, i_{1}, i_{2}, i_{3} \in\{1, \ldots, m\}$. Let us consider $r \in\{1, \ldots, m\}, P_{j}=x_{j_{1}} \cdots x_{j_{k+1}}$ with $j_{1}, \ldots, j_{k+1} \in\{1, \ldots, m\}$ with $j_{l} \neq r$ for all $l=1, \ldots, m$. Let us con- 
sider, moreover, $i_{1}, i_{2} \in\{1, \ldots, m\}$. Therefore, if $i_{1} \neq i_{2}$,

$$
\left[x_{j_{1}} \cdots x_{j_{k+1}} \frac{\partial}{\partial x_{i_{1}}}, x_{i_{1}} x_{i_{2}} \frac{\partial}{\partial x_{r}}\right]=x_{j_{1}} \cdots x_{j_{k+1}} x_{i_{2}} \frac{\partial}{\partial x_{r}}
$$

and, if $i_{1}=i_{2}$,

$$
\left[x_{j_{1}} \cdots x_{j_{k+1}} \frac{\partial}{\partial x_{i_{1}}}, x_{i_{1}} x_{i_{2}} \frac{\partial}{\partial x_{r}}\right]=2 x_{j_{1}} \cdots x_{j_{k+1}} x_{i_{1}} \frac{\partial}{\partial x_{r}} .
$$

On the other hand, for $j_{1}, \ldots, j_{k-s+1} \in\{1, \ldots, m\}$ with $j_{l} \neq r$ and $s \geq 1$,

$$
\begin{array}{r}
{\left[x_{r}^{s} x_{j_{1}} \cdots x_{j_{k-s+1}} \frac{\partial}{\partial x_{r}}, x_{r}^{2} \frac{\partial}{\partial x_{r}}\right]=} \\
2 x_{r}^{s+1} x_{j_{1}} \cdots x_{j_{k-s+1}} \frac{\partial}{\partial x_{r}}-s x_{r}^{s+1} x_{j_{1}} \cdots x_{j_{k-s+1}} \frac{\partial}{\partial x_{r}}= \\
(2-s) x_{r}^{s+1} x_{j_{1}} \cdots x_{j_{k-s+1}} \frac{\partial}{\partial x_{r}} .
\end{array}
$$

Moreover, for $s=2$ and $l \neq r$,

$$
\begin{array}{r}
{\left[x_{r}^{2} x_{j_{1}} \cdots x_{j_{k-1}} \frac{\partial}{\partial x_{r}}, x_{r} x_{l} \frac{\partial}{\partial x_{r}}\right]=} \\
x_{r}^{3} x_{j_{1}} \cdots x_{j_{k-1}} \frac{\partial}{\partial x_{r}}-2 x_{l} x_{r}^{2} x_{j_{1}} \cdots x_{j_{k-1}} \frac{\partial}{\partial x_{r}}= \\
x_{r}^{3} x_{j_{1}} \cdots x_{j_{k-1}} \frac{\partial}{\partial x_{r}}-2\left[x_{r} x_{j_{1}} \cdots x_{j_{k-1}} x_{l} \frac{\partial}{\partial x_{r}}, x_{r}^{2} \frac{\partial}{\partial x_{r}}\right],
\end{array}
$$

i.e.

$$
\begin{array}{r}
{\left[x_{r}^{2} x_{j_{1}} \cdots x_{j_{k-1}} \frac{\partial}{\partial x_{r}}, x_{r} x_{l} \frac{\partial}{\partial x_{r}}\right]+2\left[x_{r} x_{j_{1}} \cdots x_{j_{k-1}} x_{l} \frac{\partial}{\partial x_{r}}, x_{r}^{2} \frac{\partial}{\partial x_{r}}\right]=} \\
x_{r}^{3} x_{j_{1}} \cdots x_{j_{k-1}} \frac{\partial}{\partial x_{r}}
\end{array}
$$

Hence all the generators of $W(m, 0)_{k+1}$ lie in $\left[W(m, 0)_{k}, W(m, 0)_{1}\right]=$ $\left[W(m, 0)_{1}^{k}, W(m, 0)_{1}\right]=W(m, 0)_{1}^{k+1}$. It follows $W(m, 0)_{k+1}=W(m, 0)_{1}^{k+1}$ for all $k \geq 1$ and $m \geq 2$.

$5)$ Let us prove that $\left[W(m, 0)_{-1}, W(m, 0)_{1}\right]=W(m, 0)_{0}$. As before we only 
need to prove that $\left[W(m, 0)_{-1}, W(m, 0)_{1}\right] \supseteq W(m, 0)_{0}$. Since $\left[\frac{\partial}{\partial x_{i}}, x_{i}^{2} \frac{\partial}{\partial x_{j}}\right]=$ $2 x_{i} \frac{\partial}{\partial x_{j}}, W(m, 0)_{0} \subseteq\left[W(m, 0)_{-1}, W(m, 0)_{1}\right]$, i.e.

$$
W(m, 0)_{0}=\left[W(m, 0)_{-1}, W(m, 0)_{1}\right] .
$$

Let us prove that $\left[W(m, 0)_{0}, W(m, 0)_{1}\right]=W(m, 0)_{1}$. We only need to prove that $\left[W(m, 0)_{0}, W(m, 0)_{1}\right] \supseteq W(m, 0)_{0}$. Notice that

- $\left[x_{i} \frac{\partial}{\partial x_{i}}, x_{i}^{2} \frac{\partial}{\partial x_{i}}\right]=x_{i}^{2} \frac{\partial}{\partial x_{i}}$,

- $\left[x_{i} \frac{\partial}{\partial x_{i}}, x_{i}^{2} \frac{\partial}{\partial x_{j}}\right]=2 x_{i}^{2} \frac{\partial}{\partial x_{j}}$ for $i \neq j$,

- $\left[x_{i} \frac{\partial}{\partial x_{j}}, x_{j}^{2} \frac{\partial}{\partial x_{j}}\right]=2 x_{i} x_{j} \frac{\partial}{\partial x_{j}}$ for $i \neq j$

- $\left[x_{i} \frac{\partial}{\partial x_{j}}, x_{j}^{2} \frac{\partial}{\partial x_{k}}\right]=2 x_{i} x_{j} \frac{\partial}{\partial x_{k}}$.

Since all the generators of $W(m, 0)_{1}$ lie in $\left[W(m, 0)_{0}, W(m, 0)_{1}\right], W(m, 0)_{1} \subseteq$ $\left[W(m, 0)_{0}, W(m, 0)_{1}\right]$, i.e. $\left[W(m, 0)_{0}, W(m, 0)_{1}\right]=W(m, 0)_{1}$. Summarizing: $[W(m, 0), W(m, 0)]$ is clearly nonzero, $W(m, 0)$ is transitive, $\left(\mathbb{F}^{m}\right)^{*}$ is an irreducible $\mathfrak{g l}_{m}(\mathbb{F})$-module, hence $W(m, 0)_{-1}$ is an irreducible $W(m, 0)_{0^{-}}$ module, i.e. $W(m, 0)$ is irreducible, $\left[W(m, 0)_{-1}, W(m, 0)_{1}\right]=W(m, 0)_{0}$, $\left[W(m, 0)_{0}, W(m, 0)_{1}\right]=W(m, 0)_{1}$ and, for $k \geq 1$ and $m \geq 2, W(m, 0)_{k}=$ $W(m, 0){ }_{1}^{k}$. Therefore $W(m, 0)$ is simple for $m \geq 2$ by Proposition (6.7.2).

Remark 68. Note that, in the case of $W(1,0)=\operatorname{der}(\mathbb{F}[x]), x^{3} \frac{d}{d x} \in W(1,0)_{2}$ but $W(1,0)_{1}^{2}=\left[W(1,0)_{1}, W(1,0)_{1}\right]=0$ because $\left[a x^{2} \frac{d}{d x}, b x^{2} \frac{d}{d x}\right]=0$. It follows that $W(1,0)_{1}^{2} \neq W(1,0)_{2}$. Nevertheless we can show that $W(1,0)$ is also simple.

Proof. Note that $[W(1,0), W(1,0)] \neq 0$. Let now $I$ be a nonzero ideal of $W(1,0)$ and let $z$ be a nonzero element of $I$. Since $W(1,0)$ has a $\mathbb{Z}$-grading, $I=\oplus_{j \geq-1} I_{j}$ and $z=\sum_{i=-1}^{k} z_{i}$ with $z_{i} \in W(1,0)_{i}$. By transitivity $0 \neq$ $\left[z, W(1,0)_{-1}\right] \subseteq I$, therefore there exists an element $D \in W(1,0)_{-1}$ such that $0 \neq[z, D]=\sum_{i=-1}^{k}\left[z_{i}, D\right]=\sum_{i=-1}^{k-1}\left[z_{i+1}, D\right]=\sum_{i=-1}^{k-1} \tilde{z}_{i} \in I$. Iterating this argument we prove that there exists a nonzero element of $I \cap W(m, 0)_{-1}$. 
On the other hand $I \cap W(m, 0)_{-1}=W(m, 0)_{-1}$ because $\operatorname{dim} W(m, 0)_{-1}=1$. Hence $I \supseteq W(m, 0)_{-1}$. In particular, $\frac{d}{d x} \in I$. It follows that $I \supseteq W(1,0)_{k}$ for every $k \geq-1$ because $-(k+2) x^{k+1} \frac{d}{d x}=\left[x^{k+2} \frac{d}{d x}, \frac{d}{d x}\right] \in I$. Thus $I=$ $W(1,0)$.

\subsection{Example 4: the Lie superalgebra $W(0, n)$}

Let us consider the Grassmann superalgebra $\Lambda(n)$ in $n$ symbols $\xi_{1}, \ldots, \xi_{n}$. In this section we write $\xi_{i} \xi_{j}$ instead of $\xi_{i} \wedge \xi_{j}$, therefore $\xi_{i} \xi_{j}=-\xi_{j} \xi_{i}$.

Remark 69. By definition of derivation of a superalgebra, if $D$ is a derivation of $A=\Lambda(n)$, then $D(1)=0$. It follows that the images $D\left(\xi_{i}\right)$ define a derivation of $A$ completely, due to the linearity of $D$ and the Leibniz rule (6.8). In particular, the maps

$$
\begin{gathered}
\frac{\partial}{\partial \xi_{i}}: A \rightarrow A \\
\frac{\partial}{\partial \xi_{i}}\left(\xi_{j}\right)=\delta_{i j}
\end{gathered}
$$

for $i, j=1, \ldots, n$ extend uniquely to derivations of $A$.

Notation 4 . We will denote the Lie superalgebra of derivations $\operatorname{der}(\Lambda(n))$ by $W(0, n)$.

\section{Proposition 7.6.1.}

$$
W(0, n)=\left\{D=\sum_{i=1}^{n} P_{i} \frac{\partial}{\partial \xi_{i}} \mid P_{i} \in \Lambda(n)\right\}
$$

Proof. $\sum_{i=1}^{n} P_{i} \frac{\partial}{\partial \xi_{i}}$ are derivations because $\frac{\partial}{\partial \xi_{i}}$ is a derivation for every $i$. Let now $D \in W(0, n)$. Then $D\left(\xi_{i}\right)=P_{i}$ for some $P_{i} \in \Lambda(n)$. It follows that $D=\sum_{i=1}^{n} P_{i} \frac{\partial}{\partial \xi_{i}}$.

Remark 70. If we set $\operatorname{deg} \xi_{i}=1$ for $i=1, \ldots, n$, we obtain a consistent $\mathbb{Z}$-grading of $\Lambda(n)$ which induces a $\mathbb{Z}$-grading of $W(0, n): W(0, n)=$ 
$\oplus_{k \geq-1} W(0, n)_{k}$ with

$$
\begin{array}{r}
W(0, n)_{k}=\left\{D \in W(0, n) \mid D\left(\Lambda(n)_{i}\right) \subseteq \Lambda(n)_{i+k}\right\}= \\
\left\{\sum_{i} P_{i} \frac{\partial}{\partial \xi_{i}} \mid \operatorname{deg} P_{i}=k+1, i=1, \ldots, n\right\} .
\end{array}
$$

Note that $W(0, n)_{-1}=\left\langle\frac{\partial}{\partial \xi_{1}}, \ldots, \frac{\partial}{\partial \xi_{n}}\right\rangle$ and that $\left[\frac{\partial}{\partial \xi_{i}}, \frac{\partial}{\partial \xi_{j}}\right] \in W(0, n)_{-2}=0$ consistently with

$$
\frac{\partial}{\partial \xi_{i}} \frac{\partial}{\partial \xi_{j}}=-\frac{\partial}{\partial \xi_{j}} \frac{\partial}{\partial \xi_{i}}
$$

Proposition 7.6.2. 1. $W(0, n)=\oplus_{k=-1}^{n-1} W(0, n)_{k}$ is transitive.

2. The Lie algebras $W(0, n)_{0}$ and $\mathfrak{g l}_{n}(\mathbb{F})$ are isomorphic.

3. The $W(0, n)_{0}$-modules $W(0, n)_{-1}$ and $\left(\mathbb{F}^{n}\right)^{*}$ are isomorphic.

4. $W(0, n)_{k}=W(0, n)_{1}^{k}$ for $k \geq 1$.

5. $W(0, n)$ is simple for $n \geq 2$.

Proof. 1) Let us consider $a \in W(0, n)_{\geq 0}$ such that $\left[a, W(0, n)_{-1}\right]=0$. We want to prove that $a=0$. We can assume $a \in W(0, n)_{k}$, i.e. $a=\sum_{i=1}^{n} P_{i} \frac{\partial}{\partial \xi_{i}}$ with $P_{i} \in \Lambda(n)_{k+1}$. Then $0=\left[a, W(0, n)_{-1}\right]$ if and only if $0=\left[a, \frac{\partial}{\partial \xi_{j}}\right]=$ $\left[\sum_{i} P_{i} \frac{\partial}{\partial \xi_{i}}, \frac{\partial}{\partial \xi_{j}}\right]=-(-1)^{k-1} \sum_{i}\left(\frac{\partial P_{i}}{\partial \xi_{j}}\right) \frac{\partial}{\partial \xi_{i}}$ for all $j=1, \ldots, n$. Therefore $\frac{\partial P_{i}}{\partial \xi_{j}}=0$ for all $i=1, \ldots, n$. Since the relation holds for every $j=1, \ldots, n$, $P_{i}$ is a scalar. On the other hand $P_{i} \in \Lambda(n)_{k+1}$ with $k \geq 0$, hence $P_{i}=0$ because the only scalar in $\Lambda(n)_{k+1}$ with $k \geq 0$ is 0 .

2) Since $W(0, n)_{0} \subseteq W(0, n)_{\overline{0}}$ and $\left[W(0, n)_{0}, W(0, n)_{0}\right] \subseteq W(0, n)_{0}, W(0, n)_{0}$ is a Lie algebra. $W(0, n)_{0}=\left\{\sum_{i} P_{i} \frac{\partial}{\partial \xi_{i}} \mid \operatorname{deg} P_{i}=1\right\}=\left\langle\xi_{i} \frac{\partial}{\partial \xi_{j}}\right| i, j=$ $1, \ldots, n\rangle$. Let us define the following linear map:

$$
\phi: \mathfrak{g l}_{n}(\mathbb{F}) \rightarrow W(0, n)_{0}, e_{i j} \mapsto \xi_{i} \frac{\partial}{\partial \xi_{j}}
$$


Since the linear map $\phi$ is linear and surjective by construction and $\operatorname{dim} \mathfrak{g l}_{n}(\mathbb{F})=$ $\operatorname{dim} W(0, n)_{0}, \phi$ is an isomorphism of vector spaces. Moreover

$$
\begin{array}{r}
\phi([A, B])=\phi\left(\left[\sum_{i, j=1}^{n} A_{i j} e_{i j}, \sum_{k, l=1}^{n} B_{k l} e_{k l}\right]\right)= \\
\sum_{i, j=1}^{n} \sum_{k, l=1}^{n} A_{i j} B_{k l} \phi\left(\left[e_{i j}, e_{k l}\right]\right)= \\
\sum_{i, j=1}^{n} \sum_{k, l=1}^{n} A_{i j} B_{k l} \phi\left(\delta_{j k} e_{i l}-\delta_{l i} e_{k j}\right)= \\
\sum_{i, j=1}^{n} \sum_{k, l=1}^{n} A_{i j} B_{k l} \delta_{j k} \phi\left(e_{i l}\right)-\sum_{i, j=1}^{n} \sum_{k, l=1}^{n} A_{i j} B_{k l} \delta_{l i} \phi\left(e_{k j}\right)= \\
\sum_{i, j=1}^{n} \sum_{l=1}^{n} A_{i j} B_{j l} \xi_{i} \frac{\partial}{\partial \xi_{l}}-\sum_{i, j=1}^{n} \sum_{k=1}^{n} A_{i j} B_{k i} \xi_{k} \frac{\partial}{\partial \xi_{j}}
\end{array}
$$

and

$$
\begin{array}{r}
{[\phi(A), \phi(B)]=\sum_{i, j=1}^{n} \sum_{k, l=1}^{n} A_{i j} B_{k l}\left[\phi\left(e_{i j}\right), \phi\left(e_{k l}\right)\right]=} \\
\sum_{i, j=1}^{n} \sum_{k, l=1}^{n} A_{i j} B_{k l}\left[\xi_{i} \frac{\partial}{\partial \xi_{j}}, \xi_{k} \frac{\partial}{\partial \xi_{l}}\right]= \\
\sum_{i, j=1}^{n} \sum_{k, l=1}^{n} A_{i j} B_{k l}\left(\delta_{j k} \xi_{i} \frac{\partial}{\partial \xi_{l}}-\delta_{l i} \xi_{k} \frac{\partial}{\partial \xi_{j}}\right)= \\
\sum_{i, j=1}^{n} \sum_{l=1}^{n} A_{i j} B_{j l} \xi_{i} \frac{\partial}{\partial \xi_{l}}-\sum_{i, j=1}^{n} \sum_{k=1}^{n} A_{i j} B_{k i} \xi_{k} \frac{\partial}{\partial \xi_{j}} .
\end{array}
$$

Therefore $\phi$ is an isomorphism of Lie algebras.

3) $W(0, n)_{-1}$ is a $W(0, n)_{0}$-module with respect to the restriction of the adjoint action, $W(0, n)_{-1}=\left\langle\frac{\partial}{\partial \xi_{1}}, \ldots, \frac{\partial}{\partial \xi_{n}}\right\rangle$. Let us define the following linear map:

$$
\psi:\left(\mathbb{F}^{n}\right)^{*} \rightarrow W(0, n)_{-1}, e_{i}^{*} \mapsto \frac{\partial}{\partial \xi_{i}}
$$

Since the map $\psi$ is linear, surjective and $\operatorname{dim}\left(\mathbb{F}^{n}\right)^{*}=\operatorname{dim} W(0, n)_{-1}, \psi$ is an isomorphism of vector spaces. Moreover, thank to the isomorphism of Lie 
algebras defined in (7.27),

$$
\begin{array}{r}
\psi\left(\left(\sum_{i, j=1}^{n} A_{i j} \xi_{i} \frac{\partial}{\partial \xi_{j}}\right) \cdot\left(\sum_{k}^{n} b_{k} e_{k}^{*}\right)\right)=\psi\left(\left(\sum_{i, j=1}^{n} A_{i j} e_{i j}\right) \cdot\left(\sum_{k}^{n} b_{k} e_{k}^{*}\right)\right)= \\
\psi\left(-\sum_{i, j} \sum_{k} A_{i j} b_{k} \delta_{k i} e_{j}^{*}\right)=-\sum_{i, j} A_{i j} b_{i} \frac{\partial}{\partial \xi_{j}}
\end{array}
$$

and

$$
\begin{array}{r}
\left(\sum_{i, j=1}^{n} A_{i j} \xi_{i} \frac{\partial}{\partial \xi_{j}}\right) \cdot \psi\left(\sum_{k}^{n} b_{k} e_{k}^{*}\right)= \\
\left(\sum_{i, j=1}^{n} A_{i j} \xi_{i} \frac{\partial}{\partial \xi_{j}}\right) \cdot\left(\sum_{k}^{n} b_{k} \frac{\partial}{\partial \xi_{k}}\right)=\sum_{i, j=1}^{n} \sum_{k}^{n} A_{i j} b_{k}\left[\xi_{i} \frac{\partial}{\partial \xi_{j}}, \frac{\partial}{\partial \xi_{k}}\right]= \\
\sum_{i, j=1}^{n} \sum_{k}^{n} A_{i j} b_{k}\left(\xi_{i} \frac{\partial}{\partial \xi_{j}} \frac{\partial}{\partial \xi_{k}}-\delta_{i k} \frac{\partial}{\partial \xi_{j}}+\xi_{i} \frac{\partial}{\partial \xi_{k}} \frac{\partial}{\partial \xi_{i}}\right)= \\
-\sum_{i, j=1}^{n} A_{i j} b_{i} \frac{\partial}{\partial \xi_{j}} .
\end{array}
$$

Therefore $\psi$ is a homomorphism of $W(0, n)_{0}$-modules and an isomorphism of vector spaces. It follows that $\psi$ is an isomorphism of $W(0, n)_{0}$-modules.

4) $W(0, n)_{1}=\left\langle\xi_{i} \xi_{j} \frac{\partial}{\partial \xi_{k}}\right\rangle$. Let us prove that $W(0, n)_{k}=W(0, n)_{1}^{k}$ by induction on $k$. If $k=1$ there is nothing to prove. Let us suppose that $W(0, n)_{k}=$ $W(0, n)_{1}^{k}$ and let us prove that $W(0, n)_{k+1}=W(0, n)_{1}^{k+1}$. We only need to 
prove that $W(0, n)_{k+1} \subseteq W(0, n)_{1}^{k+1}$.

$$
\begin{aligned}
& {\left[\xi_{i_{1}} \cdots \xi_{i_{k+1}} \frac{\partial}{\partial \xi_{m}}, \xi_{j_{1}} \xi_{j_{2}} \frac{\partial}{\partial \xi_{l}}\right]=} \\
& \delta_{m j_{1}} \xi_{i_{1}} \cdots \xi_{i_{k+1}} \xi_{j_{2}} \frac{\partial}{\partial \xi_{l}}-\delta_{m j_{2}} \xi_{i_{1}} \cdots \xi_{i_{k+1}} \xi_{j_{1}} \frac{\partial}{\partial \xi_{l}}+ \\
& \xi_{i_{1}} \cdots \xi_{i_{k+1}} \xi_{j_{1}} \xi_{j_{2}} \frac{\partial}{\partial \xi_{m}} \frac{\partial}{\partial \xi_{l}}-(-1)^{k} \xi_{j_{1}} \xi_{j_{2}} \frac{\partial}{\partial \xi_{l}}\left(\xi_{i_{1}} \cdots \xi_{i_{k+1}}\right) \frac{\partial}{\partial \xi_{m}}- \\
& (-1)^{2 k+1} \xi_{j_{1}} \xi_{j_{2}} \xi_{i_{1}} \cdots \xi_{i_{k+1}} \frac{\partial}{\partial \xi_{l}} \frac{\partial}{\partial \xi_{m}}= \\
& \delta_{m j_{1}} \xi_{i_{1}} \cdots \xi_{i_{k+1}} \xi_{j_{2}} \frac{\partial}{\partial \xi_{l}}-\delta_{m j_{2}} \xi_{i_{1}} \cdots \xi_{i_{k+1}} \xi_{j_{1}} \frac{\partial}{\partial \xi_{l}}+ \\
& \xi_{i_{1}} \cdots \xi_{i_{k+1}} \xi_{j_{1}} \xi_{j_{2}} \frac{\partial}{\partial \xi_{m}} \frac{\partial}{\partial \xi_{l}}-(-1)^{k} \xi_{j_{1}} \xi_{j_{2}} \frac{\partial}{\partial \xi_{l}}\left(\xi_{i_{1}} \cdots \xi_{i_{k+1}}\right) \frac{\partial}{\partial \xi_{m}}+ \\
& (-1)^{2 k+1+2(k+1)} \xi_{i_{1}} \cdots \xi_{i_{k+1}} \xi_{j_{1}} \xi_{j_{2}} \frac{\partial}{\partial \xi_{m}} \frac{\partial}{\partial \xi_{l}}= \\
& \delta_{m j_{1}} \xi_{i_{1}} \cdots \xi_{i_{k+1}} \xi_{j_{2}} \frac{\partial}{\partial \xi_{l}}-\delta_{m j_{2}} \xi_{i_{1}} \cdots \xi_{i_{k+1}} \xi_{j_{1}} \frac{\partial}{\partial \xi_{l}}- \\
& (-1)^{k} \xi_{j_{1}} \xi_{j_{2}} \frac{\partial}{\partial \xi_{l}}\left(\xi_{i_{1}} \cdots \xi_{i_{k+1}}\right) \frac{\partial}{\partial \xi_{m}}
\end{aligned}
$$

with $i_{1}, \ldots i_{k+1}, j_{1}, j_{2}, m, l \in\{1, \ldots, n\}, i_{s} \neq i_{r}$ if $s \neq r$ and $j_{1} \neq j_{2}$. Therefore

$$
\begin{array}{r}
{\left[\xi_{i_{1}} \cdots \xi_{i_{k+1}} \frac{\partial}{\partial \xi_{i_{1}}}, \xi_{i_{2}} \xi_{i_{k+2}} \frac{\partial}{\partial \xi_{i_{2}}}\right]=} \\
-(-1)^{k} \xi_{i_{2}} \xi_{i_{k+2}} \frac{\partial}{\partial \xi_{i_{2}}}\left(\xi_{i_{1}} \cdots \xi_{i_{k+1}}\right) \frac{\partial}{\partial \xi_{i_{1}}}= \\
(-1)^{k} \xi_{i_{2}} \xi_{i_{k+2}} \xi_{i_{1}} \xi_{i_{3}} \cdots \xi_{i_{k+1}} \frac{\partial}{\partial \xi_{i_{1}}}= \\
-\xi_{i_{1}} \xi_{i_{2}} \cdots \xi_{i_{k+2}} \frac{\partial}{\partial \xi_{i_{1}}}
\end{array}
$$


Besides

$$
\begin{array}{r}
{\left[\xi_{i_{1}} \cdots \xi_{i_{k+1}} \frac{\partial}{\partial \xi_{i_{k+3}}}, \xi_{i_{1}} \xi_{i_{k+2}} \frac{\partial}{\partial \xi_{i_{1}}}\right]=} \\
-(-1)^{k} \xi_{i_{1}} \xi_{i_{k+2}} \frac{\partial}{\partial \xi_{i_{1}}}\left(\xi_{i_{1}} \cdots \xi_{i_{k+1}}\right) \frac{\partial}{\partial \xi_{i_{k+3}}}= \\
(-1)^{k+1} \xi_{i_{1}} \xi_{i_{k+2}} \xi_{i_{2}} \cdots \xi_{i_{k+1}} \frac{\partial}{\partial \xi_{i_{k+3}}}= \\
-\xi_{i_{1}} \cdots \xi_{i_{k+2}} \frac{\partial}{\partial \xi_{i_{k+3}}}
\end{array}
$$

Since the elements of the form $\xi_{i_{1}} \cdots \xi_{i_{k+2}} \frac{\partial}{\partial \xi_{m}}$ with $i_{j} \neq i_{j^{\prime}}$ for $j \neq j^{\prime}$ and $i_{j}, m \in\{1, \ldots, n\}$ are generators of $W(0, n)_{k+1}$ we have proven that $W(0, n)_{k+1} \subseteq\left[W(0, n)_{k}, W(0, n)_{1}\right]=\left[W(0, n)_{1}^{k}, W(0, n)_{1}\right]=W(0, n)_{1}^{k+1}$.

$5)$ Let us prove that $\left[W(0, n)_{-1}, W(0, n)_{1}\right]=W(0, n)_{0}$. As before, we only need to prove that $\left[W(0, n)_{-1}, W(0, n)_{1}\right] \supseteq W(0, n)_{0}$. We have $\left[\frac{\partial}{\partial \xi_{i}}, \xi_{j} \xi_{l} \frac{\partial}{\partial \xi_{k}}\right]=$ $\delta_{i j} \xi_{l} \frac{\partial}{\partial \xi_{k}}-\delta_{i l} \xi_{j} \frac{\partial}{\partial \xi_{k}}$. Therefore $\left[\frac{\partial}{\partial \xi_{i}}, \xi_{i} \xi_{j} \frac{\partial}{\partial \xi_{k}}\right]=\xi_{j} \frac{\partial}{\partial \xi_{k}}$ which are generators of $W(0, n)_{0}$. Now let us prove that $\left[W(0, n)_{0}, W(0, n)_{1}\right]=W(0, n)_{1}$. Note that $\left[\xi_{i} \frac{\partial}{\partial \xi_{j}}, \xi_{l} \xi_{k} \frac{\partial}{\partial \xi_{m}}\right]=\delta_{j l} \xi_{i} \xi_{k} \frac{\partial}{\partial \xi_{m}}-\delta_{j k} \xi_{i} \xi_{l} \frac{\partial}{\partial \xi_{m}}-\delta_{m i} \xi_{l} \xi_{k} \frac{\partial}{\partial \xi_{j}}$. Therefore, given $i, j, k, m \in\{1, \ldots, n\}$ with $k \neq j$ and $i \neq m,\left[\xi_{i} \frac{\partial}{\partial \xi_{j}}, \xi_{j} \xi_{k} \frac{\partial}{\partial \xi_{m}}\right]=\xi_{i} \xi_{k} \frac{\partial}{\partial \xi_{m}}$, i.e. $W(0, n)_{1} \subseteq\left[W(0, n)_{0}, W(0, n)_{1}\right]$. Summarizing: $W(0, n)$ is transitive, since $\left(\mathbb{F}^{n}\right)^{*}$ is an irreducible $\mathfrak{g l}_{n}(\mathbb{F})$-module, $W(0, n)_{-1}$ is an irreducible $W(0, n)_{0^{-}}$ module, hence $W(0, n)$ is irreducible, $W(0, n)_{0}=\left[W(0, n)_{-1}, W(0, n)_{1}\right]$, $W(0, n)_{1}=\left[W(0, n)_{0}, W(0, n)_{1}\right]$ and $W(0, n)_{k}=W(0, n)_{1}^{k}$ for $k \geq 1$. Therefore $W(0, n)$ is simple for $n \geq 2$ by Proposition (6.7.2).

Remark 71 . The Lie superalgebra of derivations $W(0,1)$ is not simple, indeed $W(0,1)=W(0,1)_{-1} \oplus W(0,1)_{0}$ and $\left[\frac{d}{d \xi}, \xi \frac{d}{d \xi}\right]=\frac{d}{d \xi}$ hence $W(0,1)_{-1}$ is a nonzero ideal properly contained in $W(0,1)$. 


\section{Bibliography}

[1] Humphreys, J. E. (1972) Introduction to Lie Algebras and RepresentationTheory, Springer-Verlag New York, Heidelberg, Berlin.

[2] Jacobson, N. (1962) Lie Algebras, Dover Publications, Inc. New York.

[3] Procesi, C. (2007) An approach to Lie theory through Invariants and Representations, Springer.

[4] Kac, V. G. (1977) Lie superalgebras, Advances in Mathematics 26, 8-96.

[5] Kac, V. G. (1968) Simple irreducible graded Lie algebras of finite growth, Math. USSR - Izvestija, Vol. 2, No. 6, pagg. 1271-1311. 